\author{
Universidade De SÃo Paulo \\ Instituto DE FísicA
}

\title{
AplichȩÃo DAs Regras de Soma DA QCD no Estudo De Possíveis Estados Moleculares
}

Jorgivan Morais Dias

Orientador(a): Profa. Dra. Marina Nielsen

Dissertação submetida ao Instituto de Física da Universidade de São Paulo, para a obtenção do título de mestre em ciências

Banca Examinadora:

$\operatorname{Prof}^{a}$. Dr ${ }^{a}$. Marina Nielsen (USP)

Prof. Dr. Fernando Silveira Navarra (USP)

$\operatorname{Prof}^{a}$. Dr ${ }^{a}$. Mirian Enriqueta Bracco (UERJ)

São Paulo, 2011. 
FICHA CATALOGRÁFICA

Preparada pelo Serviço de Biblioteca e Informação do Instituto de Física da Universidade de São Paulo

Dias, Jorgivan Morais

Aplicação das regras de soma da QCD no estudo de possíveis estados moleculares. - São Paulo, 2011.

Dissertação (Mestrado) - Universidade de São Paulo. Instituto de Física, Departamento de Física Experimental.

Orientador: Profa. Dra. Marina Nielsen

Área de Concentração: Física de Partículas

Unitermos:1.Cromodinâmica Quântica; 2. Mésons Exóticos;

3. Teoria de Campos; 4. Física de Altas Energias

USP/IF/SBI-022/2011 
"O que perturba e intimida meu espírito forte não é a certeza da morte, mas, a incerteza da vida".

(Da Costa e Silva) 


\section{Agradecimentos}

Sempre ouvi dizer que a parte mais difícil de qualquer que seja um trabalho, por menor que sejam os desafios para sua concretização (isso não o torna menos importante), é agradecer àqueles que contribuíram na longa jornada do desenho que nos tornamos (posso neste momento, garantir que isso é verdade). Por que não agradecer também aos que contribuíram negativamente, eu me pergunto, podemos aprender com eles quem exatamente não devemos ser. Em especial, inicio agradecendo a quem eu talvez, por infortúnio, esqueça de agradecer.

Agradeço àquele que é indispensável na crença da existência, elaborada pelas mãos do artesão universal: Deus. Por me emprestar até os dias de hoje, o oxigênio que respiro, o coração que pulsa, e permitir que eu enxergue na vastidão do amor, da amizade e da feliciade e até mesmo da tristeza, da revolta, da injustiça, aspectos que me fortificam.

Agradeço a Maria dos Remédios Dias de Morais, minha mãe, que me deu a profunda responsabilidade e felicidade de ser seu filho. Que tanto em momentos felizes e difíceis não permitiu em nenhum segundo, ao longo de minha vida, desanimar e, ainda hoje me mostra a saída por entre os caminhos tortuosos da vida, inspirando em mim o homem que sou. Mostrando que ideiais são como flores que nascem nos terrenos da inteligência e crescem nos vales da mente humana.

Agradeço também ao homem o qual a vida pouco permite está em família: Antônio Francisco de Morais, meu pai. O trabalho o forçou a fazer do mundo sua casa, mas, em meu coração ele fixou morada. Desbravando a vida, transforma distância em sustento. Seu caráter, coragem, sua assinatura, estão por trás da cortina de minha existência. Mostrou-me quem exatamente devo ser. A ele, palavras são mais que supérfluas para expressar minha gratidão, meu respeito e sua importância para minha vida.

À Marina Nielsen, pela paciência e por seu voto de confiança depositada em mim durante a realização deste trabalho. Pela amizade e pelos seus conselhos. Seu 
bom humor e sua maneira de agir como colega de seus alunos, em muito contribui para a profundidade da formação pessoal e acadêmica dos mesmos. Minhas sinceras desculpas pelas demoras nos trabalhos.

Agradeço à mulher que permitiu dar o primeiro passo rumo ao meu ideal, abrindo as portas para a minha jornada acadêmica. Por vezes fez o papel de mãe. Sua amizade, suas palavras de força juntamente com seu exemplo, deram a mim a centelha de coragem para não ceder às barreiras que ao longo de meu percurso na graduação foram se erguendo. Celina, a você meu muito obrigado.

À Anita Moraes, pela profunda amizade, apoio, sinceridade, inestimável ajuda e, incontestável confiança depositada em mim. A ela serei eternamente grato.

Não posso esquecer os nomes: Antonio Sales, Antonio e Marcus Damasceno pela ajuda na minha mudança para São Paulo, pelos conselhos e pelas agradáveis conversas. Ex-contemporâneos de graduação e atuais contemporâneos de pós, mas, que fique a certeza que serão eternos amigos, pelos quais tenho grande estima. A eles meu muito obrigado.

Agradeço também ao Raphael pela profunda paciência que o mesmo apresenta no dia-a-dia de nossa sala. Como eu perturbo esse cara!!... pelas discussões tanto profissionais como pessoais. Ao Ricardo Matheus, pelas valiosas discussões tanto sobre Regras de Soma como sobre outras discussões não menos importantes do que aquelas na hora do café... Ao David, grande "esclepscência", por ministrar o melhor curso sobre vinho que já tive. Pelas discussões sempre bem humoradas, reduzindo nos risos a saudade de minha família.

Aos demais professores e alunos que formam não somente um grupo de pesquisa, mas um ambiente agradável (uma espécie de família) o Grupo de Hádrons e Física Teórica (GRHAFITE): Fernando Navarra, Manoel Robilota (Obrigado pelas dicas de itinerários em São Paulo), Celso Lima, Patrícia, Érike, Bruno, Carina, André e Stefano. Ao pessoal da secretaria, Paulo, Anderson e Sérgio Korogui. Valeu pessoal...

Ao pessoal da "Confraterpingação"da Física Geral: Eduardo, Antônio Mário, Rafael, Lucas, Jônatas, David, Rodrigo. Ao João Bosco que não é o cantor!, é antes 
disso, um grande amigo. Ao pandoleiro Felipe (companheiro de festeca, quinta e breja, canto da ema, rei das batidas...), Diego e Jônathas...

Aos presepeiros e companheiros de pós, Cláudio Padilha, Fabrício e Amaury (eu nunca falei desse jeito uai!). A vocês meu muito obrigado... Ao Yul, que me apresentou a Sala São Paulo e infelizmente apresentou também o Masayuke (hehehe...). Carol e Masayuke... Valeu pessoal...

Não posso esquecer àqueles que estiveram do meu lado nos bons e maus momentos, cujas palavras amigas foram mais que motivadoras para continuar seguindo, agradeço a todos eles ao longo desses dois anos cuja amizade, desenharam meu caráter, meus caminhos, com muitas ou poucas vírgulas, e permitiram com risos e gargalhadas que eu por um momento esquecesse o stresse das disciplinas, dos tormentos da vida e as crises existenciais e me divertisse investigando e aprendendo o verdadeiro significado da vida, são eles: Tiago Adorno, Nayara Fonseca, Karine, Eliane, Antonio Sales, Érike. Meus sinceros agradecimentos por tudo...

Em especial à minha querida e doce "meiga senhorita"Thaiany Quevedo Melo, pela paciência, pelo carinho, pelo amor e pelo companheirismo. Por fazer parte de minha vida e pelo apoio creditados a mim.

À FAPESP pelo apoio financeiro. 


\section{Abstract}

Using the QCD sum rules we test if the new narrow structure, the $\mathrm{X}(4350)$ recently observed by the Belle Collaboration, can be described as a $J^{P C}=1^{-+}$ exotic $D_{s}^{*} D_{s 0}^{*}$ molecular state. We consider the contributions of condensates up to dimension eight, we work at leading order in $\alpha_{s}$ and we keep terms which are linear in the strange quark mass $m_{s}$. The mass obtained for such state is $m_{D_{s}^{*} D_{s 0}^{*}}=$ $(5.05 \pm 0.19) \mathrm{GeV}$. We also consider a molecular $1^{-+}, D^{*} D_{0}^{*}$ current and we obtain $m_{D^{*} D_{0}^{*}}=(4.92 \pm 0.08) \mathrm{GeV}$. We conclude that it is not possible to describe the $\mathrm{X}(4350)$ structure as a $1^{-+} D_{s}^{*} D_{s 0}^{*}$ molecular state. 



\section{Resumo}

Usando as Regras de Soma da QCD, testamos se a nova estrutura estreita, $X(4350)$ recentemente observada pela Colaboração Belle, pode ser descrita como um estado molecular $D_{s}^{*} D_{s 0}^{*}$ exótico $J^{P C}=1^{-+}$. Consideramos as contribuições dos condensados de dimensão oito, trabalhamos com os termos dominantes em $\alpha_{s}$ mantendo os termos lineares na massa do quark estranho $m_{s}$. A massa obtida é igual a $m_{D_{s}^{*} D_{s 0}^{*}}=(5.05 \pm 0.19) \mathrm{GeV}$. Consideramos também uma corrente molecular $1^{-+}, D^{*} D_{0}^{*}$ e obtemos $m_{D^{*} D_{0}^{*}}=(4.92 \pm 0.08) \mathrm{GeV}$. Concluímos que não é possível descrever a estrutura $X(4350)$ como um estado molecular $1^{-+} D_{s}^{*} D_{s 0}^{*}$. 



\section{Sumário}

1 Introdução 1

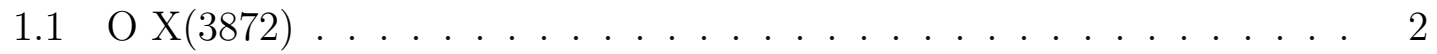

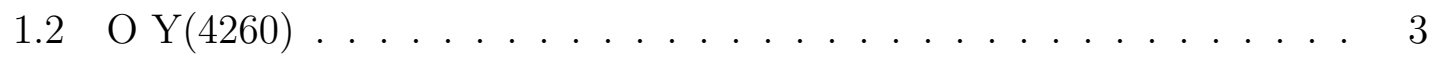

$1.3 \mathrm{O} Z^{+}(4430) \ldots \ldots \ldots \ldots \ldots$

$1.4 \mathrm{O} \mathrm{Y}(4140) \ldots \ldots \ldots \ldots \ldots \ldots$

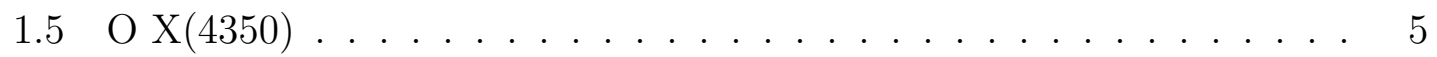

2 Regras de Soma da QCD $\quad 7$

2.1 Função de Correlação . . . . . . . . . . . . . . . . . . . . . . . 8

2.2 Expansão em Produto de Operadores - Lado da OPE . . . . . . . . . 11

2.2.1 O Propagador Perturbativo . . . . . . . . . . . 12

2.2 .2 O Propagador Não-Perturbativo . . . . . . . . . . . . . . . . . 14

2.2.3 O Condensado de Quarks . . . . . . . . . . . 15

2.2.4 O Condensado Misto . . . . . . . . . . . . . 16

2.2.5 O Condensado de Glúons - Parte Não-Fatorável . . . . . . . . 17

2.2.6 O Condensado Misto - Parte Não-Fatorável . . . . . . . . . 17

2.3 Estados Físicos Intermediários - Lado Fenomenológico . . . . . . . . . 19

2.4 A Transformada de Borel . . . . . . . . . . . . . . . . . . . 22

2.5 A Janela de Borel . . . . . . . . . . . . . . . . . . . . . . . 24

2.5.1 Determinando o Máximo da Massa de Borel . . . . . . . . . . 24

2.5.2 Determinando o Mínimo da Massa de Borel . . . . . . . . . . 25 
2.6 O Princípio da Dualidade Quark-Hádron e a Determinação dos Parâmetros Hadrônicos . . . . . . . . . . . . . . . . . . . . 26

3 O Estado X(4350) 29

3.1 Os Números Quânticos e a Corrente . . . . . . . . . . . . . . 29

3.1.1 Conjugação de Carga e a Paridade da Corrente . . . . . . . . 32

3.2 Lado da OPE . . . . . . . . . . . . . . . . . . . . . . . 33

3.2.1 Diagramas Perturbativos . . . . . . . . . . . . 35

3.2.2 Diagramas dos Condensados de Quarks . . . . . . . . . . 40

3.2.3 Diagramas dos Condensados de Glúons . . . . . . . . . . . . . 41

3.2.4 Diagramas do Condensado Misto . . . . . . . . . . . . . . . 42

3.2.5 Diagramas dos Condensados de 4-Quarks . . . . . . . . . . 45

3.2.6 Condensados de Dimensão $8 \ldots$. . . . . . . . . . . . . . 46

3.3 Lado Fenomenológico . . . . . . . . . . . . . . . . . . . . . . . . . . . 48

3.4 Regra de Soma . . . . . . . . . . . . . . . . . . . . 49

3.5 Resultados Numéricos . . . . . . . . . . . . . . . . . . 50

$\begin{array}{llr}4 & \text { Conclusões } & 57\end{array}$

$\begin{array}{ll}\text { A Propagadores } & 59\end{array}$

A.1 Propagadores associados com quarks leves . . . . . . . . . . . 59

A.2 Propagadores associados com quarks pesados . . . . . . . . . . . . 60

B Algumas Relações Usadas em Regras de Soma 63

B.1 Calculando os Traços . . . . . . . . . . . . . . . . . . 63

B.2 Transformadas de Fourier . . . . . . . . . . . . . . . . . 64

B.3 Álgebra das Matrizes de Dirac . . . . . . . . . . . . . . . . . 64

B.4 Parametrização de Schwinger $(\gamma) \ldots \ldots$. . . . . . . . . . 66

$\begin{array}{ll}\text { C Integrais } & 67\end{array}$

C.1 Integrais no Momento . . . . . . . . . . . . . . 67

C.2 A Integral $I_{n} \ldots \ldots \ldots \ldots$. . . . . . . . . . . . 68 
C.3 A Integral $I_{n m k l} \ldots \ldots \ldots \ldots$. . . . . . . . . . . . . . . 69

C.4 A Integral $I_{\lambda}^{n} \ldots \ldots \ldots \ldots \ldots \ldots$

C.5 Cálculo dos Diagramas Perturbativos . . . . . . . . . . . . . 71 



\section{Lista de Figuras}

1.1 Produção do $Y(4140)$ a partir da colisão próton-antipróton. . . . . . . 5

2.1 Diagrama representando a amplitude calculada por uma função de dois pontos. $q$ e $\bar{q}$, representam um quark e anti-quark, respectivamente. 8

2.2 Diagrama representando a amplitude calculada por uma função de três pontos. $q$ e $\bar{q}$, representam um quark e anti-quark, respectivamente. 9

2.3 Diagrama representando a contribuição não-fatorável no cálculo da função de correlação. . . . . . . . . . . . . . . . . . . . . . . 18

2.4 A linha contínua representa a variação da contribuição do Pólo $(P)$ com a Massa de Borel ao quadrado para $\sqrt{s_{0}}=5.3 \mathrm{GeV}$, enquanto a linha pontilhada representa a contribuição do contínuo $C$. Note que as contribuições se igualam quando $M^{2}$ for igual a $3.74 \mathrm{GeV}^{2}$, portanto, esse valor, para este caso, será o valor para $M_{\max }$. . . . . 25

2.5 Comportamento da série devido ao acréscimo das contribuições relativas de cada um dos condensados. $s_{0}$, é o limiar do threshold para o qual o cálculo foi realizado $\left(\sqrt{s_{0}}=5.3 \mathrm{GeV}\right)$. Abaixo de $M^{2}$ igual a $3.2 \mathrm{GeV}^{2}$, é fácil ver que a série apresenta problemas de convergência. 26

3.1 Diagrama representando um estado molecular formado por dois mésons, as duas linhas superiores representam um méson formado por um quark $c$ e um anti-quark $s$ enquanto as duas linhas inferiores representam um méson formado por um quark $s$ e um anti-quark $c$. . . 35 
3.2 Diagramas perturbativos. Note que, o símbolo $\times$ presente nas linhas do quark e do anti-quark s, representam a correção na massa dos mesmos. . . . . . . . . . . . . . . . 36

3.3 Diagramas associados aos Condensados de Quarks. . . . . . . . . . . 40

3.4 Diagramas associados aos Condensados de Glúons. . . . . . . . . . . 41

3.5 Diagramas fatorizáveis associados ao Condensados Misto. . . . . . . . 43

3.6 Diagramas Não-Fatorizáveis associados ao Condensado Misto. . . . . . 44

3.7 Diagramas associados aos Condensados de 4-Quarks. . . . . . . . . . 45

3.8 Diagramas associados à dimensão 8 da OPE. . . . . . . . . . . . . . . 47

3.9 Comportamento da série devido ao acréscimo das contribuições relativas de cada um dos condensados. O cálculo foi realizado para $\sqrt{s_{0}}=5.45 \mathrm{GeV}$.

3.10 Massa calculada para o estado $D_{s}^{*} D_{s 0}^{*}$ em função da Massa de Borel ao quadrado $M^{2}$, para diferentes valores de $\sqrt{s_{0}}$. O símbolo $\times$ em cada curva representa os valores máximo e mínimo da Janela de Borel. 52

3.11 Contribuição do Pólo versus Contínuo para $\sqrt{s_{0}}=5.3 \mathrm{GeV}$. . . . . 53

3.12 Convergência da OPE para o caso no qual estamos supondo o estado descrito pela molécula $D^{*} D_{0}^{*}$, em função de $M^{2}$. . . . . . . . 54

3.13 Relação Pólo versus Contínuo para $\sqrt{s_{0}}=5.4 \mathrm{GeV}$. . . . . . . . . 55

3.14 Massa associada ao méson $X(4350)$ calculada supondo uma corrente molecular $D^{*} D_{0}^{*}$, como função do parâmetro que determina o limiar do contínuo $\sqrt{s_{0}}$, para $\sqrt{s_{0}}=5.2 \mathrm{GeV}$ (linha contínua), $\sqrt{s_{0}}=5.3 \mathrm{GeV}$ (linha pontilhada), $\sqrt{s_{0}}=5.4 \mathrm{GeV}$ (linha ponto-tracejada) e $\sqrt{s_{0}}=$ $5.5 \mathrm{GeV}$ (linha tracejada). . . . . . . . . . . . . 56 56 


\section{Lista de Tabelas}

3.1 Mésons $D$ associados com as respectivas correntes de acordo com o sinal da paridade e o valor do spin . . . . . . . . . . . . . . . 30

3.2 Limite superior da Janela de Borel $M_{\max }^{2}$ associado com alguns valores de $\sqrt{s_{0}}$ para a Regra de Soma do $X(4350)$ supondo-o um estado exótico $1^{-+} \ldots \ldots \ldots \ldots \ldots \ldots \ldots$ 



\section{Capítulo 1}

\section{Introdução}

As interações fortes de partículas fundamentais tem sido satisfatoriamente descritas por uma teoria de gauge não-abeliana chamada Cromodinâmica Quântica (QCD). Por isso, acredita-se que ela seja a teoria básica da física de hádrons. A QCD possui duas importantes características, a saber: liberdade assintótica e o confinamento. A liberdade assintótica permite-nos descrever fenômenos por meio de quarks e glúons considerando-os aproximadamente livres em processos de altas energias, onde o acoplamento torna-se pequeno o suficiente para que se possa aplicar técnicas de teoria de perturbação. Nesse regime perturbativo (altas energias) a teoria está bem consolidada e testada com uma precisão de $1 \%$.

Por outro lado, no regime de baixas energias, o acoplamento se torna grande, devido ao caráter não abeliano da interação, de tal maneira que os métodos de teoria de perturbação se tornam inviáveis. Por conta disso, o estado fundamental da QCD não é vazio e sim, um condensado de quarks, anti-quarks e glúons acentuando ainda mais as dificuldades dos problemas em baixas energias. Com isso, torna-se difícil a determinação do espectro hadrônico nesse regime de energia.

Partindo disso, vários modelos baseados na QCD foram propostos a fim de tentar resolver o problema do espectro. O mais bem sucedido deles, é o modelo de quarks proposto por Murray Gell-Mann [1] e também por George Zweig [2].

Os hádrons, de acordo com o modelo de quarks, podem ser classificados de acordo 
com o conteúdo de quarks em sua estrutura. Assim, hádrons formados por um par quark-antiquark são chamados de Mésons. Por outro lado, os Bárions (próton e nêutron) são formados por três quarks.

No cenário atual da física de partículas, a grande maioria dos hádrons são muito bem descritos pelo modelo de quarks, muito embora nenhum cálculo em QCD mostre que não é possível haver estados hadrônicos com diferentes conteúdos de quarks daqueles anteriormente mencionados. Por conseguinte, acredita-se que existem estados hadrônicos de quatro quarks (tetraquarks), estados sem nenhum quark em sua estrutura (Glueballs) e estados com glúons excitados (híbridos). Qualquer partícula (estado hadrônico) cujo conteúdo de quarks seja diferente de quark-antiquark ou três quarks, é denominada de exóticos.

Dados recentes sobre a espectroscopia do charmonium, revelam a possibilidade de estados que podem ser descritos por moléculas. Desde que um próton e um nêutron formam o dêuteron, acredita-se que mésons também podem formar moléculas. Esses dados foram obtidos nas chamadas B-factories, que inicialmente foram construídas para a investigação da violação de CP em decaimento de mésons B. As B-factories são colisores de elétron-pósitron, onde uma grande quantidade de mésons $B$ e $\bar{B}$ são produzidas na aniquilação daqueles. Esses novos estados observados, apesar de estarem na região de massa do charmonium, não se encaixam no espectro de massa dos estados $c \bar{c}$ calculados usando o modelo de quarks. Em seguida, vamos citar alguns desses estados.

\section{$1.1 \quad \mathrm{O} X(3872)$}

Em 2003, a colaboração Belle anunciou a descoberta do méson $X(3872)$ no processo $B^{+} \rightarrow X(3872) K^{+} \rightarrow J / \psi \pi^{+} \pi^{-} K^{+}$. Esse méson, é o mais bem estudado dos estados associados $c \bar{c}$. Quatro experimentos o confirmaram [3], com a massa de $3872 \pm 0.6 \mathrm{MeV}$ e uma largura muito pequena: $\Gamma<2.3 \mathrm{MeV}$. Logo após sua descoberta, o $X(3872)$ pareceu ser um candidato para o estado $\psi_{2}\left({ }^{3} D_{2}\right)$, mas as transições radiativas desse estado para o $\chi_{c}$ não foram observadas. 
Ambas as colaborações, Belle [4] e BaBar [5], observaram o decaimento $X(3872) \rightarrow$ $\gamma J / \psi$, indicando que o méson $X(3872)$ possui $C=+$ como conjugação de carga. Em contraposição, os estados convencionais do charmonium possuem $C=-$, assim, o $X(3872)$ não pode ser identificado como um estado convencional do charmonium.

Dados mais recentes de estudos combinando informações angulares e propriedades cinemáticas do par $\pi^{+} \pi^{-}$, realizados pelas colaborações Belle e BaBar, favorecem o conjunto de números quânticos $1^{++}$para o $X(3872)$. Na Referência [6] os autores mostraram que além de $1^{++}$, os números $2^{-+}$também são compatíveis com os dados. Entretanto, um cenário desfavorável a respeito dos números $1^{++}$foi anunciado pela colaboração BaBar observando o decaimento $J / \psi \omega$ [7]. A possibilidade de $2^{-+}$, também é desfavorecida pela observação do decaimento em $\psi(2 S) \gamma$ e ainda pela observação do decaimento em $D^{0} \bar{D}^{0} \pi^{0}$, ambas pelas colaborações Belle e BaBar $[8,9]$.

\section{$1.2 \quad \mathrm{O} Y(4260)$}

O méson $Y$ (4260), descoberto pela colaboração BaBar em 2005, por meio de processos ISR (Initial State Radiation), é um outro exemplo de supostos estados convencionais do charmonium. Esse estado com massa em torno de $4300 \mathrm{MeV}$, foi observado no processo $e^{+} e^{-} \rightarrow \gamma_{I S R} J / \psi \pi^{+} \pi^{-}$, com uma largura $90 \mathrm{MeV}$.

Em um processo ISR, o pósitron ou o elétron irradia um fóton e, em seguida, o par elétron-pósitron se aniquila. Em tal processo, há produção de estados numa região de massa do charmonium com números quânticos $J^{P C}=1^{--}$, isso ocorre quando a energia do centro de massa do fóton irradiado se encontra numa faixa entre 4000 a $5000 \mathrm{MeV}$. O méson Y (4260) é produzido em processos ISR, entretanto, nessa região de massa (4000 e $5000 \mathrm{MeV}$ ), os estados convencionais do charmonium estão todos bem estabelecidos, são eles: $\psi(4040)$ : estado $3^{3} S_{1}, \psi(4160)$ : estado $2^{3} D_{1}$ e $\psi(4415)$ : estado $4^{3} S_{1}$. Suas massas estão de acordo com os modelos de potencial. Isso motivou alguns autores a considerar o $Y(4260)$ como sendo um estado de tetraquarks [10]. Na referência [11], foram consideradas diferentes opções de estrutura, baseadas em 
tetraquarks e estados moleculares, para o estudo desse méson. Tal estudo revelou que é possível explicar a massa do $Y(4260)$ considerando que ele seja um estado molecular $D_{0} \overline{D^{*}}$.

\section{$1.3 \mathrm{O} Z^{+}(4430)$}

O $Z^{+}$(4430), observado em 2007 pela colaboração Belle [12] no decaimento $B^{+} \rightarrow K Z^{+}(4430) \rightarrow K \psi^{\prime} \pi^{+}$, é o mais intrigante de todos já que, sendo um estado carregado, não pode ser um estado $c \bar{c}$ puro. A massa e a largura observadas desse estado são $M=(4433 \pm 5) \mathrm{MeV}$ e $\Gamma=(45 \pm 20) \mathrm{MeV}$. Como o conteúdo mínimo desse estado é $c \bar{c} u \bar{d}$, ele é o principal candidato para um estado de muitos quarks.

Se os mésons $X(3872), Z^{+}(4430)$ e $Y(4260)$ são de fato estados moleculares, então, muitas outras moléculas deverão existir. Um estudo sistemático desses possíveis estados moleculares, e sua observação experimental, forneceriam um novo campo de testes para a QCD, dentro das configurações de muitos quarks. Na referência [13] as regras de soma da QCD foram usadas para calcular a massa de um possível estado molecular $D_{s} D^{*}$. Essa predição, e a predição de outros possíveis estados moleculares estranhos, é de particular interesse para experimentos que deverão investigar com precisão muito maior o espectro de energia do charmonium, como o PANDA (na máquina próton-antipróton no laboratório FAIR).

\section{$1.4 \mathrm{O} \mathrm{Y}(4140)$}

A colaboração CDF (Collider Detector at Fermilab) observou recentemente o estado $Y(4140)$ no canal de decaimento $B^{+} \rightarrow Y(4140) K^{+} \rightarrow J / \psi \phi K^{+}$. A massa e a largura dessa estrutura são, $M=(4143 \pm 2.9 \pm 1.2) \mathrm{MeV}$ e $\Gamma=\left(11.7_{-5.0}^{+8.3}\right) \mathrm{MeV}$ [14]. Na Referência [15], os autores o interpretaram como um parceiro molecular do $Y(3930)$, observado pelas colaborações Belle e BaBar próximo do threshold $J / \psi \omega$ [16]. Eles concluíram que o $Y(4140)$ é provavelmente um estado molecular $D_{s}^{*} \bar{D}_{s}^{*}$ com $J^{P C}=0^{++}$ou $2^{++}$. Na Referência [17], o $Y(4140)$ foi interpretado como um 
charmonium híbrido exótico com $J^{P C}=1^{-+}$.

\section{$1.5 \mathrm{O} X(4350)$}

Usando modelos de Potencial, os autores da ref. [18], sugeriram que se o $Y(4140)$ fosse um estado molecular $D_{s}^{*+} D_{s}^{*-}$, ele deveria ser observado no processo de dois fótons. Seguindo tal sugestão, a colaboração Belle procurou observar o estado Y(4140) no canal $\gamma \gamma \rightarrow \phi J / \psi$. No entanto, como podemos observar pela Fig. 1.1, a colaboração Belle detectou uma estrutura estreita em $4.35 \mathrm{GeV}$ no espectro de massa do $J / \psi \phi$, ao invés do estado $Y(4140)$. A massa e a largura desse estado, chamado $X(4350)$, são: $M=\left(4350.6_{-5.1}^{+4.6}\right) \mathrm{MeV}$ e $\Gamma=\left(13.3_{-9.1}^{+7.9} \pm 4.1\right) \mathrm{MeV}[19]$.

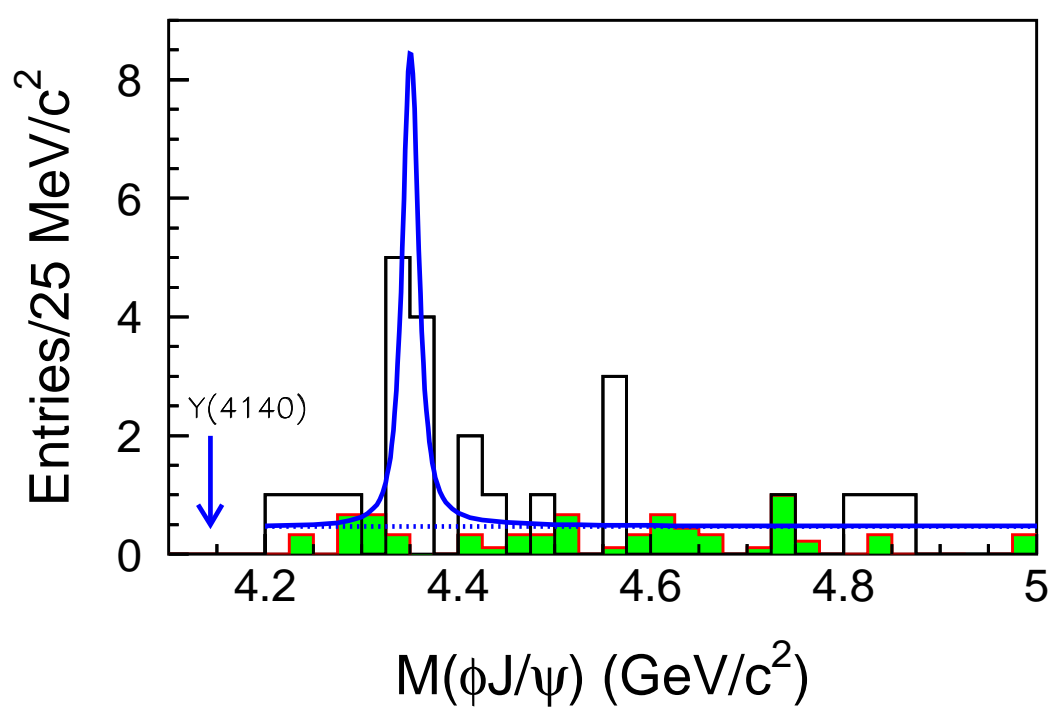

Figura 1.1: Produção do $Y(4140)$ a partir da colisão próton-antipróton.

Em nosso trabalho [20] usamos a técnica das Regras de Soma da QCD para calcular a massa do estado $X(4350)$ considerando-o como um estado molecular exótico.

Esta dissertação está organizada da seguinte maneira: no Capítulo 2, introduzimos a técnica das Regras de Soma ressaltando suas vantagens e limitações, apresentando os principais parâmetros envolvidos no uso desta técnica para o cálculo de massa. A descrição de nossos resultados para o cálculo da massa do estado $X(4350)$ 
supondo-o como um estado molecular exótico, será apresentada em detalhes no Capítulo 3. Por fim, o Capítulo 4 é dedicado à nossas considerações finais. 


\section{Capítulo 2}

\section{Regras de Soma da QCD}

As Regras de Soma da QCD consistem numa técnica de cálculo, que trata de aspectos não perturbartivos da QCD, desenvolvida pelos físicos Shifman, Vainshtein e Zakharov[21]. Uma das vantagens dessa técnica é o fato dela ser analítica, possibilitando o cálculo de algumas propriedades dos hádrons, como por exemplo, determinação da massa do estado fundamental, constantes de decaimentos e fatores de forma.

O método consiste na comparação de duas diferentes abordagens da função de correlação. Tal comparação é possível devido ao princípio da Dualidade QuarkHádron. Assim, através dessa igualdade, podemos calcular as propriedades listadas anteriormente. Por outro lado, o método das regras de soma é limitado por aproximações realizadas no cálculo da função de correlação. Dessa forma, temos que estudá-las caso a caso, para determinarmos seus limites de validade.

Este capítulo está organizado da seguinte maneira: Na seção 1.1, apresentamos a definição da função de correlação, ressaltando sua importância nas Regras de Soma. Nas seções 1.2 e 1.3, descrevemos o Lado Fenomenológico e o formalismo de Expansão em Produto de Operadores, que são as duas abordagens da função de correlação usadas no cálculo de Regras de Soma. Nas seções 1.4 e 1.5, descrevemos o papel da Transformada de Borel e a importância da determinação de uma região do espaço da Massa de Borel, chamada janela de Borel, que possa garantir a confiabilidade 
dos resultados. Finalmente, na seção 1.6, mostramos como calcular os parâmetros hadrônicos usando o Princípio da Dualidade Quark-Hádron.

\subsection{Função de Correlação}

A função de correlação, ou correlator, desempenha um papel fundamental nas regras de soma da QCD, pois é a partir dela que calculamos as amplitudes de diagramas como o da Figura abaixo:

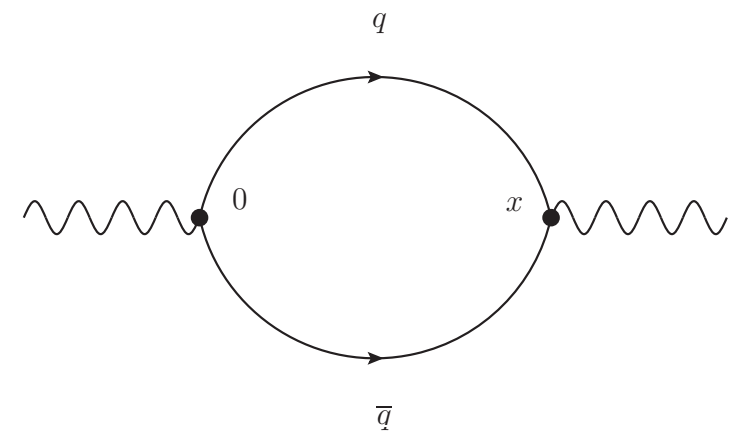

Figura 2.1: Diagrama representando a amplitude calculada por uma função de dois pontos. $q$ e $\bar{q}$, representam um quark e anti-quark, respectivamente.

A Figura 2.1 apresenta um diagrama, em que os estados inicial e final são hádrons, associado a uma função de correlação também conhecida por função de dois pontos, que no caso de mésons vetoriais é definida como:

$$
\Pi_{\mu \nu}(q)=i \int d^{4} x e^{i q x}\left\langle 0\left|T\left[j_{\mu}(x) j_{\nu}^{\dagger}(0)\right]\right| 0\right\rangle
$$

onde, $j_{\mu}(x)$ é a corrente escrita em termos dos campos de quarks contendo os números quânticos do hádron. Para mésons escalares $j(x)$ é igual a $j(x)=\overline{q_{a}}(x) q_{b}(x)$, onde $a$ e $b$ são índices de cor e $q$ é o campo do quark. No caso de mésons vetoriais temos $j_{\mu}=\overline{q_{a}}(x) \gamma_{\mu} q_{b}(x)$ e $j=\bar{q}(x) i \gamma_{5} q(x)$ para mésons pseudo-escalares. Funções de correlação de dois pontos são utilizadas para calcular massas de hádrons e constantes de acoplamento. 
Considere agora a Figura 2.2, na qual temos uma terceira linha em $y$. Tais diagramas estão associados com funções de correlação de três pontos que tem utilidade no cálculo de fatores de forma.

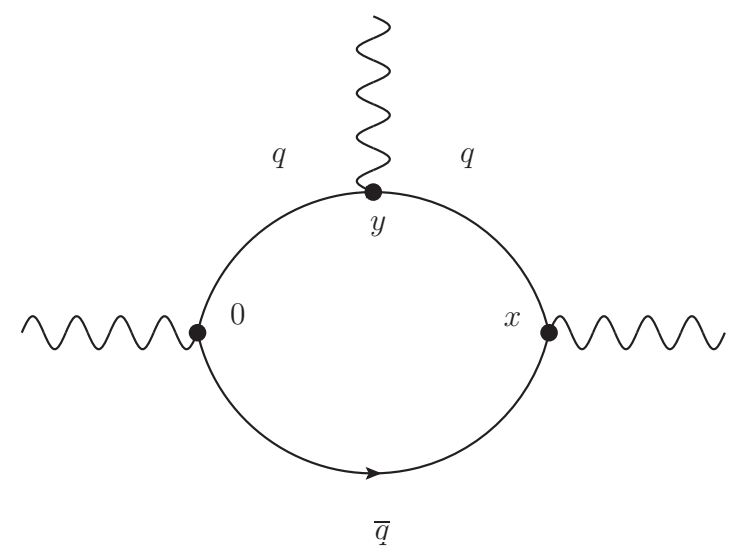

Figura 2.2: Diagrama representando a amplitude calculada por uma função de três pontos. $q$ e $\bar{q}$, representam um quark e anti-quark, respectivamente.

No caso do diagrama da Figura 2.2, a função de correlação é definida como

$$
\Pi(q)=i \int d^{4} x d^{4} y e^{i p_{1} x} e^{-i p_{2} y}\left\langle 0\left|T\left[j_{1}(x) j_{2}^{\dagger}(y) j_{3}^{\dagger}(0)\right]\right| 0\right\rangle
$$

onde $p_{1}$ é o momento do hádron representado pela corrente $j_{1}$ e $p_{2}$ é o momento do hádron representado pela corrente $j_{2}$.

Sabemos que a constante de acoplamento da QCD, $\alpha_{s}=\frac{g_{s}}{4 \pi^{2}}$, varia com a escala de energia. Em processos hadrônicos nos quais alguns dos quarks e glúons envolvidos são altamente virtuais, $\alpha_{s}$ é suficientemente pequena e, com isso, podemos usar os métodos perturbativos. Tais processos são chamados de "processos duros" e acontecem numa alta escala de energia (acima de $1 \mathrm{GeV}$ ). Isso está relacionado à curtas distâncias e, segundo a QCD, nesse regime os quarks e glúons propagam-se livremente (liberdade assintótica), por conseguinte, a função de correlação, é escrita em termos dos propagadores livres. Isto é, aplicando o teorema de Wick ao elemento 
de matriz na Equação (2.1), supondo a corrente de um méson vetorial, temos

$$
\begin{aligned}
\left\langle 0\left|T\left[j_{\mu}(x) j_{\nu}(0)\right]\right| 0\right\rangle & =\left\langle 0\left|T\left[\overline{q_{a}}(x) \gamma_{\mu} q_{a}(x) \overline{q_{b}}(0) \gamma_{\nu} q_{b}(0)\right]\right| 0\right\rangle \\
& =\left(\gamma_{\mu}\right)_{i j}\left(\gamma_{\nu}\right)_{k l}\left\langle 0_{p}\left|T\left[\overline{q_{a}}(x)_{i} q_{b}(0)_{l}\right]\right| 0_{p}\right\rangle \times \\
& \times\left\langle 0_{p}\left|T\left[q_{a}(x)_{j} \overline{q_{b}}(0)_{l}\right]\right| 0_{p}\right\rangle+ \\
& +\left(\gamma_{\mu}\right)_{i j}\left(\gamma_{\nu}\right)_{k l}\left\langle 0_{p}\left|\overline{q_{a}}(x)_{i} q_{b}(0)_{l}\right| 0_{p}\right\rangle\left\langle 0\left|: q_{a}(x)_{j} \overline{q_{b}}(0)_{k}:\right| 0\right\rangle+\ldots,
\end{aligned}
$$

onde $\left|0_{p}\right\rangle$ e " :: " representam o vácuo perturbativo e o ordenamento normal, respectivamente. Os termos da equação acima com $\left\langle 0_{p}|\ldots| 0_{p}\right\rangle$, são definidos como o propagador de quark livre enquanto que o segundo termo $\left\langle 0\left|: q_{a}(x)_{j} \overline{q_{b}}(0)_{k}:\right| 0\right\rangle$ é nulo, no vácuo perturbativo. Por outro lado, como (2.3) será avaliada no vácuo da QCD, implica que o termo com "::" introduz as informações não-perturbativas do vácuo da QCD. Podemos levar isso em conta por meio de uma expansão em produtos de operadores (OPE - Operators Product Expansion) [28]. Nas Regras de Soma, chamamos a descrição por meio dessa expansão em produto de operadores de Lado da OPE.

Por outro lado, em vez de escrevermos a função de correlação em termos de campos de quarks, podemos considerar os hádrons como se fossem partículas fundamentais. Como conseqüência disso, as correntes presentes no correlator são interpretadas como operadores de campo dos hádrons. Tal descrição da função de correlação, é chamada no método das Regras de Soma, de Lado Fenomenológico.

Com isso, podemos entender a importância da função de correlação nas Regras de Soma. A razão é que a mesma função pode ser considerada em dois níveis diferentes, a saber: em termos de campos de quarks e glúons (Lado da OPE) ou considerando os graus de liberdade sendo puramente hadrônicos (Lado Fenomenológico). É nessa prescrição, chamada de Princípio da Dualidade Quark-Hádron, que as Regras de Soma estão apoiadas. Tal princípio, afirma que podemos descrever um hádron tanto a nível de quarks quanto a nível hadrônico.

Nas próximas seções, entraremos em detalhes a respeito dessas duas diferentes abordagens sobre a função de correlação, mencionadas anteriormente, ou seja, o Lado Fenomenológico e o Lado da OPE. 


\subsection{Expansão em Produto de Operadores - Lado da OPE}

Considere o produto temporalmente ordenado dado por (2.4)

$$
i \int d x e^{i q x} T\left\{j(x) j^{\dagger}(0)\right\}
$$

Observe que as correntes $j(x)$ e $j^{\dagger}(0)$ não estão definidas em um único ponto. Contudo, é possível expandir o produto temporalmente ordenado de duas correntes em termos de um conjunto completo de operadores locais como

$$
i \int d x e^{i q x} T\left\{j(x) j^{\dagger}(0)\right\}=\sum_{d} C_{d} \hat{O}_{d}(0)
$$

onde $C_{d}$ são os coeficientes de Wilson ao passo que $\hat{O}_{d}$ são operadores locais compostos a partir de campos de quarks e glúons. A expansão na Equação (2.5) é chamada de expansão do produto de operadores (OPE) [28]. Toda a informação associada com a física de curto alcance (parte perturbativa), é dada pelos coeficientes de Wilson. Tais termos podem ser calculados pela teoria perturbativa. Por outro lado, os efeitos não-perturbativos, correspondentes à física de longo alcance, estão contidos em $\hat{O}_{d}$. A soma é realizada sobre a dimensão do operador local, indicado pelo índice $d$. Deste modo, para $d=0$ (dimensão zero), o operador será a própria identidade, ou seja, $\hat{O}_{0}(0)=\hat{1}$. Para $d=3,4$ e 5 temos, respectivamente,

$$
\begin{gathered}
\hat{O}_{3}=\bar{q} q \\
\hat{O}_{4}=G_{\mu \nu}^{k} G^{k \mu \nu} \\
\hat{O}_{5}=\bar{q} \sigma_{\mu \nu} \frac{\lambda^{a}}{2} G_{\mu \nu}^{k} q,
\end{gathered}
$$

onde $\sigma_{\mu \nu}=\frac{i}{2}\left[\gamma_{\mu}, \gamma_{\nu}\right], \gamma_{\mu}$ são as matrizes de Dirac, $\lambda^{a}$ são as matrizes de Gell-Man e $G_{\mu \nu}$ é o tensor de campo dos glúons. Os valores esperados no vácuo de (2.6), (2.7) e (2.8), são os condensados de quarks, condensado de glúons e condensado de quark-glúon, respectivamente. 
Os condensados oriundos da OPE são parâmetros puramente não-perturbativos e, por conseguinte, não podem ser calculados analiticamente. Para calculá-los, devemos recorrer a métodos indiretos. Por exemplo, para calcularmos o valor numérico de $\langle\bar{q} q\rangle$, usamos a hipótese de Corrente Axial Parcialmente Conservada - PCAC (do inglês Partially Conserved Axial Current) [22]. Isto é,

$$
\langle\bar{q} q\rangle=-\frac{m_{\pi}^{2} f_{\pi}^{2}}{2\left(m_{u}+m_{d}\right)},
$$

onde $m_{\pi}$ e $f_{\pi}$ são, respectivamente, a massa e a constante de decaimento do píon. $m_{u}$ e $m_{d}$ são as massas dos quarks $u$ e $d$. Deste modo, inserindo em (2.9) os valores $m_{u}+m_{d} \cong 14 \mathrm{MeV}, m_{\pi}=138 \mathrm{MeV}$ e $f_{\pi}=132 \mathrm{MeV}$, resulta

$$
\langle\bar{q} q\rangle=-(0,228 \mathrm{GeV})^{3} .
$$

Podemos seguir um raciocínio análogo para obtermos os valores numéricos para os demais condensados.

Na seção (2.1), obtemos (2.3) aplicando o teorema de Wick ao elemento de matriz na Equação (2.1), onde o primeiro termo daquela equação representa o propagador livre dos quarks enquanto o segundo termo é nulo na teoria perturbativa. Entretanto, na teoria não-perturbativa, é o segundo termo de (2.3) que dá origem às contribuições não-perturbativas e não-fatorizáveis do vácuo da QCD. Essas contribuições são os condensados mencionados anteriormente. A partir de agora, vamos dedicar as próximas seções à descrição das contribuições perturbativas, não-perturbativas e não-fatorizáveis, apresentando o cálculo do propagador completo utilizado nas Regras de Soma da QCD.

\subsubsection{O Propagador Perturbativo}

De acordo com o que foi dito na seção (2.1), a curtas distâncias a constante de acoplamento da QCD é suficientemente pequena, o que nos permite fazer uso da teoria perturbativa. Assim, o cálculo do propagador perturbativo é dado pela fórmula de Gell-Man Low [23]:

$$
S_{a b}^{(p)}(x)=\left\langle 0_{p}\left|T\left\{q_{a}(x) \bar{q}_{b}(0) e^{i \int d^{4} z \mathcal{L}_{i n t}(z)}\right\}\right| 0_{p}\right\rangle
$$


onde $\mathcal{L}_{\text {int }}(z)$ é a lagrangiana de interação quark-glúon sendo igual a

$$
\mathcal{L}_{\text {int }}(z)=g_{s} \bar{q}^{a}(z) \gamma^{\mu} A_{\mu}^{n} \tau_{a b}^{n} q^{b}(z)
$$

onde $g_{s}$ é a constante de acoplamento, $\tau_{a b}$ são os geradores do grupo $S U(3), A_{\mu}$ é o campo do glúon com $n$ variando de 1 até 8. Expandindo a exponencial em (2.11), resulta em

$$
S_{a b}^{(p)}(x)=\left\langle 0_{p}\left|T\left\{q_{a}(x) \bar{q}_{b}(0)\right\}\right| 0_{p}\right\rangle+i \int d^{4} z\left\langle 0_{p}\left|T\left\{q_{a}(x) \bar{q}_{b}(0) \mathcal{L}_{i n t}(z)\right\}\right| 0_{p}\right\rangle+\ldots
$$

Além disso, se chamarmos $S_{a b}^{(0)}(x) \equiv\left\langle 0_{p}\left|T\left\{q_{a}(x) \bar{q}_{b}(0)\right\}\right| 0_{p}\right\rangle$ e o segundo termo como $S_{a b}^{(1)}(x) \equiv i \int d^{4} z\left\langle 0_{p}\left|T\left\{q_{a}(x) \bar{q}_{b}(0) \mathcal{L}_{\text {int }}(z)\right\}\right| 0_{p}\right\rangle$, podemos reescrever (2.13) da seguinte maneira:

$$
S_{a b}^{(p)}(x)=S_{a b}^{(0)}(x)+S_{a b}^{(1)}(x)+\ldots
$$

O primeiro termo no lado direito de (2.14) representa o propagador de quark livre dado por,

$$
S_{a b}^{(0)}(x)=i \delta_{a b} \int \frac{d^{4} p}{(2 \pi)^{4}}\left(\frac{\not p+m_{q}}{p^{2}-m_{q}^{2}+i \epsilon}\right) e^{-i p x},
$$

enquanto que o segundo termo está associado ao vértice de interação entre quarks e glúons, sendo igual a [24]

$$
S_{a b}^{(1)}=-\frac{i}{4} g_{s} \tau_{a b}^{n} G_{\mu \nu}^{n}(0) \int \frac{d^{4} p}{(2 \pi)^{4}}\left[\frac{\sigma^{\mu \nu} \not d+\not p \sigma_{\mu \nu}+2 m_{q} \sigma^{\mu \nu}}{\left(p^{2}-m_{q}^{2}+i \epsilon\right)^{2}}\right] e^{-i p x},
$$

onde $G_{\mu \nu}^{n}$ é dado por $G_{\mu \nu}^{n}=\partial_{\mu} A^{\nu}-\partial_{\nu} A^{\mu}+g_{s} f_{j k}^{n} A_{\mu}^{j} A_{\nu}^{k}$. Se a corrente considerada contém quarks leves podemos, através de uma transformada de Fourier, escrever o propagador no espaço das configurações expandindo em primeira ordem a massa do quark. Com isso, (2.15) e (2.16) podem ser reescritos da seguinte maneira:

$$
\begin{gathered}
S_{a b}^{(0)}(x)=i \delta_{a b} \int \frac{d^{4} p}{(2 \pi)^{4}}\left(\frac{\not p+m_{q}}{p^{2}+i \epsilon}\right) e^{-i p x} \\
S_{a b}^{(1)}=-\frac{i}{4} g_{s} \tau_{a b}^{n} G_{\mu \nu}^{n}(0) \int \frac{d^{4} p}{(2 \pi)^{4}}\left[\frac{\sigma^{\mu \nu} \not p+\not \sigma_{\mu \nu}+2 m_{q} \sigma^{\mu \nu}}{\left(p^{2}+i \epsilon\right)^{2}}\right] e^{-i p x} .
\end{gathered}
$$

Usando os resultados dado no Apêndice X:

$$
\int d^{4} p \frac{\not p e^{-i p x}}{p^{2}+i \epsilon}=\not x \frac{8 \pi^{2}}{x^{4}}
$$




$$
\int d^{4} p \frac{e^{-i p x}}{p^{2}+i \epsilon}=i \frac{4 \pi^{2}}{x^{2}}
$$

(2.17) resulta em

$$
S_{a b}^{(0)}=\delta_{a b}\left(i \frac{\not}{2 \pi^{2} x^{4}}-\frac{m_{q}}{4 \pi^{2} x^{2}}\right) .
$$

Por outro lado, usando as transformadas calculadas no Apêndice X

$$
\begin{gathered}
\int d^{4} p \frac{\not p e^{-i p x}}{\left(p^{2}+i \epsilon\right)^{2}}=\not x \frac{2 \pi^{2}}{x^{2}}, \\
\int d^{4} p \frac{e^{-i p x}}{\left(p^{2}+i \epsilon\right)^{2}}=-i \pi^{2} \ln \left(-x^{2}\right),
\end{gathered}
$$

obtemos,

$$
S_{a b}^{(1)}=-i \frac{g_{s} \tau_{a b}^{n} G_{\mu \nu}^{n}}{2^{5} \pi^{2} x^{2}}\left[\sigma^{\mu \nu} \not x+\not x \sigma^{\mu \nu}\right]
$$

Portanto, somando (2.21) com (2.24), obtemos o propagador perturbativo $S_{a b}^{(p)}$ em primeira ordem na constante de acoplamento

$$
S_{a b}^{(p)}=\delta_{a b}\left(i \frac{\not x}{2 \pi^{2} x^{4}}-\frac{m_{q}}{4 \pi^{2} x^{2}}\right)-i \frac{g_{s} \tau_{a b}^{n} G_{\mu \nu}^{n}}{2^{5} \pi^{2} x^{2}}\left[\sigma^{\mu \nu} \not x+\not x \sigma^{\mu \nu}\right] .
$$

O próximo passo, é analisar o produto normal dos operadores de quarks que aparece no segundo termo de $(2.3),\left\langle 0\left|: q_{a}(x)_{j} \overline{q_{b}}(0)_{k}:\right| 0\right\rangle$, pois, como foi dito anteriormente, tal termo é responsável pelo surgimento de contribuições não-pertubartivas. O propagador completo utilizado nas Regras de Soma da QCD será a soma dos propagadores perturbativo e não-perturbativo.

\subsubsection{O Propagador Não-Perturbativo}

Os condensados que surgem do segundo termo de (2.3), são devidos a dois tipos de contribuições, a saber: contribuições fatorizáveis e não-fatorizáveis. Estes últimos, surgem devido aos termos que resultam do produto entre termos perturbativos e não-perturbativos. Iniciemos o cálculo do propagador pelas contribuições fatoráveis.

Fazendo uma expansão de $q_{a}(x)$ em torno de $x=0$, resulta em

$$
q_{a}(x)=q_{a}(0)+\left.x_{\mu} \partial^{\mu} q_{a}(x)\right|_{x=0}+\left.\frac{1}{2} x_{\mu} x_{\nu} \partial^{\mu} \partial^{\nu} q_{a}(x)\right|_{x=0}+\ldots
$$


Trabalhando no gauge do ponto fixo, ou seja, $x_{\mu} A^{\mu}=0$ podemos substituir as derivadas $\partial_{\mu}$ por derivadas covariantes $D_{\mu}$, onde $D_{\mu}=\partial_{\mu}-i g_{s} A_{\mu}$. Assim, podemos reescrever 2.26 da seguinte maneira:

$$
q_{a}(x)=q_{a}(0)+\left.x_{\mu} D^{\mu} q_{a}(x)\right|_{x=0}+\left.\frac{1}{2} x_{\mu} x_{\nu} D^{\mu} D^{\nu} q_{a}(x)\right|_{x=0}+\ldots .
$$

Podemos então, obter as contribuições não-perturbativas inserindo (2.27) no produto normal presente no segundo termo de (2.3), assim, obtemos

$$
\begin{aligned}
\langle 0|: q(x) \bar{q}(0):| 0\rangle & =\left\langle 0\left|: q_{a}(0) \bar{q}_{b}(0):\right| 0\right\rangle+x_{\mu}\left\langle 0\left|: D^{\mu} q_{a}(x)\right|_{x=0} \bar{q}_{b}(0): \mid 0\right\rangle+ \\
& +x_{\mu} x_{\nu} \frac{1}{2}\left\langle 0\left|: D^{\mu} D^{\nu} q_{a}(x)\right|_{x=0} \bar{q}_{b}(0): \mid 0\right\rangle+\ldots
\end{aligned}
$$

onde $|0\rangle$ representa o vácuo da QCD. Deste modo, cada termo de (2.28) é identificado como um condensado. A seguir, discutiremos cada um deles separadamente.

\subsubsection{O Condensado de Quarks}

O primeiro termo da série dada por (2.28) é chamado de condensado de quarks. Definindo-o como, $S_{a b}^{\alpha \beta}$, temos

$$
S_{a b}^{\alpha \beta}=\left\langle 0\left|: q_{a}^{\alpha}(0) \bar{q}_{b}^{\beta}(0):\right| 0\right\rangle=N \delta_{a b} \delta^{\alpha \beta},
$$

onde $\alpha$ e $\beta$ são índices espinoriais e $N$ é somente um fator de normalização. Para determiná-lo, multipliquemos ambos os lados da equação acima por $\delta_{b a} \delta^{\beta \alpha}$, portanto,

$$
\delta_{b a} \delta^{\beta \alpha}\left\langle 0\left|: q_{a}^{\alpha}(0) \bar{q}_{a}^{\beta}(0):\right| 0\right\rangle=N \delta_{a b} \delta^{\alpha \beta} \delta_{b a} \delta^{\beta \alpha} .
$$

Sabendo que $\delta_{a b} \delta^{\alpha \beta} \delta_{b a} \delta^{\beta \alpha}=12$ e, definindo $\delta^{\beta \alpha}\left\langle 0\left|: q^{\alpha}(0) \bar{q}^{\beta}(0):\right| 0\right\rangle \equiv\langle\bar{q} q\rangle$, podemos isolar $N$ e substituir seu resultado em (2.29), obtendo assim, o propagador para o condensado de quarks cuja expressão será dada por,

$$
S_{a b}=-\frac{\delta_{a b}}{12}\langle\bar{q} q\rangle
$$

Consideremos agora, o segundo termo de (2.28) que será definido como sendo $S_{a b}^{\mu \alpha \beta}$, portanto,

$$
S_{a b}^{\mu \alpha \beta}=\left\langle 0\left|: D^{\mu} q_{a}^{\alpha}(x)\right|_{x=0} \bar{q}_{b}^{\beta}(0): \mid 0\right\rangle=N \delta_{a b}\left(\gamma^{\mu}\right)^{\alpha \beta}
$$


Multiplicando por $\delta_{b a}\left(\gamma^{\mu}\right)^{\beta \alpha}$ os dois membros da igualdade acima, resulta em

$$
\delta_{b a}\left(\gamma^{\mu}\right)^{\beta \alpha}\left\langle 0\left|: D^{\mu} q_{a}^{\alpha}(x)\right|_{x=0} \bar{q}_{b}^{\beta}(0): \mid 0\right\rangle=N \delta_{a b}\left(\gamma^{\mu}\right)^{\alpha \beta} \delta_{b a}\left(\gamma^{\mu}\right)^{\beta \alpha}
$$

onde $\gamma^{\mu}$ é a matriz de Dirac. De posse das igualdades $\delta_{a b}\left(\gamma^{\mu}\right)^{\alpha \beta} \delta_{b a}\left(\gamma^{\mu}\right)^{\beta \alpha}=48 \mathrm{e}$ $\gamma^{\mu} D_{\mu}=\not D$, obtemos a expressão de $N$

$$
N=-\frac{\langle\bar{q} \not D q\rangle}{48}
$$

onde definimos $\left\langle 0\left|: \gamma^{\mu} D_{\mu} q_{a}^{\alpha}(x)\right|_{x=0} \bar{q}_{b}^{\beta}(0): \mid 0\right\rangle \equiv\langle\bar{q} \not D q\rangle$. Por outro lado, sabemos que

$$
\not D q_{a}^{\alpha}=-i m_{q} q_{a}^{\alpha}
$$

desse modo, decorre daí que $N=\frac{i m_{q}\langle\bar{q} q\rangle}{48}$. Assim, substituindo $N$ em (2.32), obtemos

$$
S_{a b}^{\mu}=\delta_{a b} \frac{i m_{q}}{48}\langle\bar{q} q\rangle \nsim
$$

Onde (2.36) pode ser interpretado como o termo de correção de massa.

\subsubsection{O Condensado Misto}

O termo $\left\langle 0\left|: D^{\mu} D^{\nu} q_{a}^{\alpha}(x)\right|_{x=0} \bar{q}_{b}^{\beta}(0): \mid 0\right\rangle$ em (2.28) é chamado de Condensado Misto. Seguindo um racioncínio análogo ao anterior, escrevemos

$$
S_{a b}^{\mu \nu \alpha \beta}=\left\langle 0\left|: D^{\mu} D^{\nu} q_{a}^{\alpha}(x)\right|_{x=0} \bar{q}_{b}^{\beta}(0): \mid 0\right\rangle=N \delta_{a b} \delta^{\alpha \beta} g^{\mu \nu} .
$$

Multiplicando ambos os membros da igualdade acima por $\delta_{b a} \delta^{\beta \alpha} g_{\mu \nu}$ obtemos,

$$
\delta_{b a} \delta^{\beta \alpha} g_{\mu \nu}\left\langle 0\left|: D^{\mu} D^{\nu} q_{a}^{\alpha}(x)\right|_{x=0} \bar{q}_{b}^{\beta}(0): \mid 0\right\rangle=N \delta_{a b} \delta^{\alpha \beta} g^{\mu \nu} \delta_{b a} \delta^{\beta \alpha} g_{\mu \nu}
$$

Se definirmos $\left\langle 0\left|: D^{\mu} D^{\nu} q_{a}^{\alpha}(x)\right|_{x=0} \bar{q}_{b}^{\beta}(0): \mid 0\right\rangle \equiv\left\langle\bar{q} D^{2} q\right\rangle$, resolvemos (2.38) em $N$, obtendo,

$$
N=-\frac{\left\langle\bar{q} D^{2} q\right\rangle}{48}
$$

Por outro lado, usando a relação $D^{2} q=\frac{1}{2} g_{s} \sigma G q[25]$, obtemos $\left\langle\bar{q} D^{2} q\right\rangle=\frac{1}{2}\left\langle\bar{q} g_{s} \sigma G q\right\rangle$. Deste modo, substituindo, esta última relação na expressão (2.39), em seguida inserindo (2.39) em (2.37), encontramos

$$
S_{a b}^{\mu \nu}=-\frac{g^{\mu \nu} \delta_{a b}}{96}\left\langle\bar{q} g_{s} \sigma G q\right\rangle,
$$


que é o propagador associado ao condensado misto.

As próximas contribuições não-perturbativas ao propagador das Regras de Soma da QCD são provenientes de contribuições não-fatoráveis. Eles estão associados aos condensados de Glúons e também ao condensado Misto.

\subsubsection{O Condensado de Glúons - Parte Não-Fatorável}

Em baixas energias o termo não-fatorável que dá origem ao chamado Condensado de Glúons, surge da interação entre o quark $q$ com o quark externo $q$ '. Segundo a referência [24], a contribuição para o propagador não-perturbativo associado com o condensado de glúons é dada por,

$$
S_{a b}^{\left\langle G^{2}\right\rangle}=-\frac{i \tau_{a b}^{n}}{3.2^{9} \cdot \pi^{2} \cdot x^{2}} g_{s} G_{\mu \nu}^{A}\left[\not x \sigma_{\mu \nu}+\sigma_{\mu \nu} \not x\right]
$$

\subsubsection{O Condensado Misto - Parte Não-Fatorável}

Existe ainda um outro tipo de contribuição para o propagador não-perturbativo denominado Condensado Misto. Note que, para o condensado misto há também uma contribuição fatorável, descrita anteriormente. Entretanto, considerando somente a contribuição relativa à parte não-fatorável, proveniente do condensado de quarks e glúons, temos o termo dado pela equação abaixo:

$$
\left\langle 0\left|: q^{a}(x) g_{s} G_{\mu \nu}^{n} \bar{q}^{b}(0):\right| 0\right\rangle
$$

Observe que temos um fator do tipo $g_{s} G_{\mu \nu}^{n}$. Tal fator surge devido à presença de uma linha do tipo mostrada pela Figura 2.3 no cálculo do correlator. Isso, de certa forma, induz um valor esperado no vácuo numa outra linha de quark. São justamente esses termos que dão origem às contribuições não-fatoráveis. Substituindo (2.26) em (2.42), em primeira ordem, resulta em

$$
\left\langle 0\left|: q_{\alpha}^{a}(x) g_{s} G_{\mu \nu}^{n} \bar{q}_{\beta}^{b}(0):\right| 0\right\rangle=N\left(\sigma_{\mu \nu}\right)_{\alpha \beta} \tau_{a b}^{\alpha \beta}
$$




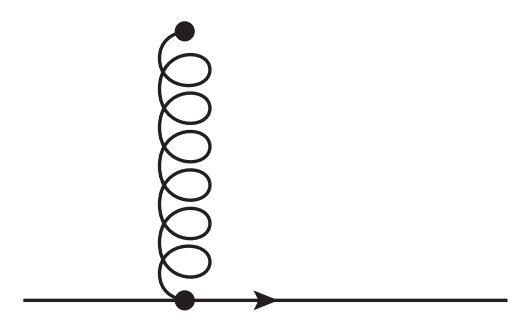

Figura 2.3: Diagrama representando a contribuição não-fatorável no cálculo da função de correlação.

Multiplicando os membros da equação acima por $\tau_{b a}^{\beta \alpha}\left(\sigma_{\mu \nu}\right)_{\beta \alpha}$ e, além disso, definindo $\left\langle 0\left|: q_{\alpha}^{a}(x) g_{s} G_{\mu \nu}^{n} \bar{q}_{\beta}^{b}(0):\right| 0\right\rangle \equiv\left\langle\bar{q} g_{s} \sigma G q\right\rangle$ encontramos,

$$
N=-\frac{1}{3.2^{6}}\left\langle\bar{q} g_{s} \sigma G q\right\rangle
$$

isso implica que,

$$
\left\langle 0\left|: q^{a}(x) g_{s} G_{\mu \nu}^{n} \bar{q}^{b}(0):\right| 0\right\rangle=-\frac{\tau_{a b}^{n}}{3.2^{6}}\left\langle\bar{q} g_{s} \sigma G q\right\rangle \sigma_{\mu \nu},
$$

que é a contribuição em primeira ordem para o condensado misto (não-fatorável). Considerando agora, termos de segunda ordem em (2.26), obtemos

$$
\left\langle 0\left|: D_{\rho} q_{\alpha}^{a}(x)\right|_{x=0} g_{s} G_{\mu \nu}^{n} \bar{q}_{\beta}^{b}(0): \mid 0\right\rangle=N\left(\gamma_{\rho} \sigma_{\mu \nu}+\sigma_{\mu \nu} \gamma_{\rho}\right)_{\alpha \beta} \tau^{n a b}
$$

Contraindo com $\left(\gamma_{\rho} \sigma^{\mu \nu}+\sigma^{\mu \nu} \gamma_{\rho}\right)^{\beta \alpha} \tau^{n b a}$, implica que

$$
N=-\frac{1}{3.2^{8}}\left\langle\bar{q} g_{s} G \not D q\right\rangle .
$$

Portanto, substituindo o valor de $N$ em (2.46) sabendo que $\not D q=-i m_{q} q$, encontra$\operatorname{mos}$

$$
\left\langle 0\left|: D_{\rho} q_{\alpha}^{a}(x)\right|_{x=0} g_{s} G_{\mu \nu}^{n} \bar{q}_{\beta}^{b}(0): \mid 0\right\rangle=\frac{i m_{q} \tau^{n a b}}{3.2^{8}}\left\langle\bar{q} g_{s} \sigma G q\right\rangle\left[\not 火 \sigma_{\mu \nu}+\sigma_{\mu \nu} \not x\right],
$$

onde $\not x$ aparece devido ao termo $\frac{1}{2} x^{\rho}$ proveniente da expansão (2.26). Assim, o propagador associado com o condensado misto devido à contribuição não-fatorável, pode ser escrito como

$$
S_{a b}^{\mu \nu}(x)=\frac{i \tau_{a b}^{n}}{3.2^{6}}\left[i \sigma_{\mu \nu}+\frac{m_{q}}{4}\left(\not \sigma_{\mu \nu}+\sigma_{\mu \nu} \not\right)\right]\left\langle\bar{q} g_{s} \sigma G q\right\rangle
$$


Finalmente, podemos escrever o propagador completo utilizado no cálculo do lado da OPE. Assim, somando todas as contribuições, temos

$$
\begin{aligned}
S_{a b}^{q}(x) & =\delta_{a b}\left(i \frac{\not x}{2 \pi^{2} x^{4}}-\frac{m_{q}}{4 \pi^{2} x^{2}}\right)-i \frac{g_{s} \tau_{a b}^{n} G_{\mu \nu}^{n}}{2^{5} \pi^{2} x^{2}}\left[\sigma^{\mu \nu} \not x+\not 火 \sigma^{\mu \nu}\right]+i \frac{\delta_{a b}}{12}\langle\bar{q} q\rangle\left[i+\frac{m_{q}}{4} \not x\right] \\
& \left.-\frac{x^{2} \delta_{a b}}{96}\left\langle\bar{q} g_{s} \sigma G q\right\rangle+\frac{i \tau_{a b}^{n}}{3.2^{6}}\left[i \sigma_{\mu \nu}+\frac{m_{q}}{4}\left(\not \sigma_{\mu \nu}+\sigma_{\mu \nu} \not\right\rangle\right)\right]\left\langle\bar{q} g_{s} \sigma G q\right\rangle .
\end{aligned}
$$

\subsection{Estados Físicos Intermediários - Lado Feno- menológico}

Na seção (2.1), foi dito que podemos entender o comportamento da função de correlação, inserindo um conjunto completo de estados físicos intermediários entre os operadores, que neste caso, são operadores de criação e aniquilação do hádron descrito pela corrente considerada. Tal abordagem é chamada de Lado Fenomenológico. Por questão de simplicidade, vamos considerar um méson escalar. Deste modo, o correlator é definido como

$$
\Pi(q)=i \int d^{4} x e^{i q x}\left\langle 0\left|T\left[j(x) j^{\dagger}(0)\right]\right| 0\right\rangle .
$$

Usando a definição de produto temporalmente ordenado, a equação (2.51) pode ser reescrita da seguinte maneira,

$$
\Pi(q)=i \int d^{4} x e^{i q x}\left\{\left\langle 0\left|\theta\left(x_{o}\right)\left[j(x) j^{\dagger}(0)\right]\right| 0\right\rangle+\left\langle 0\left|\theta\left(-x_{o}\right)\left[j^{\dagger}(0) j(x)\right]\right| 0\right\rangle\right\} .
$$

Agora, vamos assumir que o conjunto de mésons formados pela corrente $j$ seja um conjunto completo, isto é,

$$
\hat{1}=\sum_{k}^{\infty}|k\rangle\langle k| .
$$

Substituindo (2.53) entre as correntes de (2.52), obtemos,

$$
\begin{aligned}
\Pi(q)=\Pi^{f e n}(q) & =i \int d^{4} x e^{i q x} \sum_{k}\left\{\theta\left(x_{0}\right)\langle 0|j(x)| k\rangle\left\langle k\left|j^{\dagger}(0)\right| 0\right\rangle+\right. \\
& \left.+\theta\left(-x_{0}\right)\left\langle 0\left|j^{\dagger}\right| k\right\rangle\langle k|j(x)(0)| 0\rangle\right\} .
\end{aligned}
$$


Por outro lado, considere o operador $U$, com as seguintes propriedades:

$$
\begin{gathered}
\hat{U}(a)|k\rangle=e^{i p_{k} a}|k\rangle ; \\
\hat{U} \hat{U}^{-1}=\hat{1} \\
\hat{U}(a) j(x) \hat{U}^{\dagger}(a)=j(x+a) .
\end{gathered}
$$

Tal operador é chamado de operador de translação. Usando estas propriedades podemos reescrever os termos de (2.54) da seguinte maneira:

$$
\theta\left(x_{0}\right)\left\langle 0\left|\hat{U}^{-1}(-x) \hat{U}(-x) j(x) \hat{U}^{-1}(-x) \hat{U}(-x)\right| k\right\rangle=\langle 0|j(0)| k\rangle e^{-i p_{k} x} .
$$

Além disso, do cálculo acima, implica também que

$$
\langle k|j(x)| 0\rangle=e^{i p x}\left\langle k\left|j^{\dagger}(0)\right| 0\right\rangle .
$$

Portanto, substituindo (2.58) e (2.59) em (2.54), obtemos:

$$
\Pi^{f e n}(q)=i \int d^{4} x e^{i q x}\left[\theta\left(x_{0}\right) e^{i p x}+\theta\left(-x_{0}\right) e^{-i p x}\right] \sum_{k}^{\infty}\langle 0|j(0)| k\rangle\left\langle k\left|j^{\dagger}(0)\right| 0\right\rangle .
$$

De posse da identidade dada por

$$
\int_{0}^{\infty} d s \int d^{4} p \theta\left(p_{0}\right) \delta\left(p^{2}-s\right) \delta\left(p-p_{k}\right)=1,
$$

podemos reescrever (2.60) da seguinte maneira:

$$
\begin{aligned}
\Pi^{f e n}(q) & =i \int d^{4} x e^{i q x}\left[\theta\left(x_{0}\right) e^{-i p x}+\theta\left(-x_{0}\right) e^{i p x}\right] \int_{0}^{\infty} d s \times \\
& \times \int d^{4} p \delta\left(p_{0}\right) \delta\left(p^{2}-s\right) \sum_{k}\langle 0|j(0)| k\rangle\left\langle k\left|j^{\dagger}(0)\right| 0\right\rangle \delta^{4}\left(p-p_{k}\right) .
\end{aligned}
$$

Além disso, definindo $\sum_{k}\langle 0|j(0)| k\rangle\left\langle k\left|j^{\dagger}(0)\right| 0\right\rangle \delta^{4}\left(p-p_{k}\right) \equiv \frac{2 \pi}{(2 \pi)^{4}} \rho\left(p^{2}\right)$, decorre daí que

$$
\begin{aligned}
\Pi^{f e n}(q) & =\int d^{4} x e^{i q x} \int_{0}^{\infty} d s \rho(s) \int \frac{d^{4} p}{(2 \pi)^{3}}\left[i \theta\left(x_{0}\right) e^{-i p x} \theta\left(p_{0}\right) \delta\left(p^{2}-s\right)+\right. \\
& \left.+i \theta\left(-x_{0}\right) e^{i p x} \theta\left(p_{0}\right) \delta\left(p^{2}-s\right)\right] .
\end{aligned}
$$

Podemos identificar a integral

$$
\int \frac{d^{4} p}{(2 \pi)^{3}}\left[i \theta\left(x_{0}\right) e^{-i p x} \theta\left(p_{0}\right) \delta\left(p^{2}-s\right)+i \theta\left(-x_{0}\right) e^{i p x} \theta\left(p_{0}\right) \delta\left(p^{2}-s\right)\right]
$$


como o propagador de Feynman para um méson escalar $\Delta_{F}(x ; s)$, deste modo, $(2.63)$ assume a forma

$$
\Pi^{f e n}(q)=\int d^{4} x e^{i q x} \int_{0}^{\infty} d s \rho(s) \Delta_{F}(x ; s) .
$$

Entretanto, podemos também reescrever o propagador de Feynman $\Delta_{F}(x ; s)$ como

$$
\Delta_{F}(x ; s)=\int \frac{d^{4} p}{(2 \pi)^{4}} \frac{e^{-i p x}}{s-p^{2}-i \epsilon} .
$$

Por conseguinte, substituindo a equação acima em (2.65) e, resolvendo as integrais em $p$ e $x$, no limite em que $\epsilon \rightarrow 0$, encontramos

$$
\Pi^{f e n}(q)=\int_{0}^{\infty} d s \frac{\rho(s)}{s-q^{2}},
$$

onde $\rho$ é chamada de densidade espectral.

Um ponto muito importante a ressaltar neste momento, devido a observações experimentais, é que usualmente, $\rho(s)$ pode ser separada em um pólo bem definido, representando a massa do estado fundamental da partícula e numa série de estados excitados que representam um contínuo de ressonâncias de massas superiores à do pólo. Assim,

$$
\rho(s)=\lambda^{2} \delta\left(s-m^{2}\right)+\rho^{c o n t}(s)
$$

onde $\lambda$ é uma constante que fornece uma medida do acoplamento do estado com a corrente, isto é, $\langle 0|j| H\rangle=\lambda$, e $|H\rangle$ é o estado hadrônico.

Substituindo, (2.68) na Equação (2.67), podemos separar as contribuições do pólo e do contínuo na expressão da função de correlação para o Lado Fenomenológico, portanto,

$$
\Pi^{f e n}(q)=\frac{\lambda^{2}}{m^{2}-q^{2}}+\int_{s_{0}}^{\infty} d s \frac{\rho^{\text {cont }}(s)}{s-q^{2}} .
$$

Para introduzirmos um número mínimo de parâmetros no cálculo, costuma-se aproximar $\rho^{\text {cont }}(s)=\Theta\left(s-s_{0}\right) \rho^{O P E}(s)$, onde $s_{0}$ é um parâmetro, o qual indica que para valores de $s$ maiores que $s_{0}$, há somente contribuições do contínuo de ressonâncias. Portanto, ele é denominado de limiar do contínuo ou threshold. deste modo, podemos reescrever (2.69) da seguinte maneira:

$$
\Pi^{f e n}(q)=\frac{\lambda^{2}}{m^{2}-q^{2}}+\int_{s_{0}}^{\infty} d s \frac{\rho^{O P E}(s)}{s-q^{2}} .
$$


É esta equação que será comparada com a função de correlação calculada pelo Lado da OPE.

Na seção 2.1, comentamos que o principal papel que a função de correlação desempenha nas Regras de Soma da QCD, é a possibilidade de realizarmos o cálculo seguindo duas abordagens diferentes. O Princípio da Dualidade Quark-Hádron nos assegura, de certa forma, que essas duas abordagens são equivalentes. Entretanto, para que possamos usar tal princípio e, assim, podermos comparar a equação (2.69) com $\Pi(q)$ calculado pela OPE, definida como $\Pi^{O P E}(q)$, devemos escrever esta última como uma relação de dispersão, isto é,

$$
\Pi^{O P E}(q)=\frac{1}{\pi} \int_{s_{\min }}^{\infty} d s \frac{\operatorname{Im}[\Pi(s)]}{s-q^{2}}+\ldots
$$

onde os termos seguintes representados por "...", são os termos de subtração. Na equação (2.71), podemos definir uma função densidade espectral $\rho^{O P E}(s) \equiv \frac{1}{\pi} \operatorname{Im}[\Pi(s)]$. Deste modo, (2.71) pode ser reescrita como

$$
\Pi^{O P E}(q)=\int_{s_{m i n}}^{\infty} d s \frac{\rho^{O P E}(s)}{s-q^{2}} .
$$

Portanto, é essa expressão que usaremos para comparação com o Lado Fenomenológico.

\subsection{A Transformada de Borel}

Neste ponto, parece razoável utilizarmos o Princípio da Dualidade Quark-Hádron já que temos as expressões da função de correlação calculada nos Lados da OPE e Fenomenológico. Entretanto, note que o cálculo de todos os termos da OPE é impraticável. Em um dado momento temos que truncar a série e, além disso, garantir sua convergência. Contudo, para que o truncamento seja possível, as contribuições dos termos de dimensões mais altas devem ser suficientemente pequenas para que se possa justificar sua não consideração na expansão. No Lado Fenomenológico, introduzimos aproximações ao assumirmos que a densidade espectral pode ser separada em um pólo mais um contínuo de estados excitados. Além disso, estamos 
interessados no estado fundamental, isto é, na contribuição do pólo. Assim, devemos suprimir a contribuição dos estados excitados, para que o resultado seja suficientemente dominado pelo estado de mais baixa energia.

Portanto, para que ambas as descrições sejam de fato equivalentes, devemos suprimir tanto a contribuição dos termos de ordem mais alta na OPE como a contribuição dos estados excitados no Lado Fenomenológico. Isso pode ser feito através da aplicação de uma transformada chamada de Transformada de Borel. Ela é definida da seguinte maneira [27]:

$$
\beta\left[\Pi\left(Q^{2}\right)\right]=\Pi\left(M^{2}\right)=\lim _{\substack{Q^{2}, n \rightarrow \infty \\ \frac{Q^{2}}{n}=M^{2}}} \frac{\left(Q^{2}\right)^{n+1}}{n !}\left(-\frac{\partial}{\partial Q^{2}}\right)^{n} \Pi\left(Q^{2}\right),
$$

onde $Q^{2}=-q^{2}$ é o momento no espaço euclidiano e $M^{2}$ é uma variável, que surge devido à aplicação da transformada, chamada de Massa de Borel. Seguem abaixo, alguns exemplos de transformadas de Borel.

$$
\begin{gathered}
\beta\left[\left(Q^{2}\right)^{k}\right]=0, \quad \text { para } k>0 ; \\
\beta\left[\frac{1}{\left(Q^{2}\right)^{k}}\right]=\frac{1}{(k-1) !}\left(\frac{1}{M^{2}}\right)^{k-1} ; \\
\beta\left[\frac{1}{s+Q^{2}}\right]=e^{-s / M^{2}} .
\end{gathered}
$$

Aplicando a Transformada de Borel ao correlator do Lado Fenomenológico, resulta

$$
\beta\left[\Pi^{f e n}\left(Q^{2}\right)\right]=\Pi\left(M^{2}\right)=\lambda^{2} e^{-m^{2} / M^{2}}+\int_{s_{0}}^{\infty} d s \rho^{O P E}(s) e^{-s / M^{2}} .
$$

Note que, a densidade espectral associada às contribuições dos estados excitados, após a transformada, está multiplicada por uma exponencial. Assim, à medida em que $s$ cresce, o segundo termo de (2.77) torna-se menos importante, suprimindo os estados excitados.

Pelo Lado da OPE, os coeficientes de ordem mais alta são potências, $k$, da dimensão do operador, isto é, do tipo $1 /\left(Q^{2}\right)^{k}$. Assim, o uso da transformada de Borel introduz, de acordo com 1.75 , um fator igual a $1 /(k-1)$ !, reduzindo a importância dos termos de ordem mais alta na OPE. 
Como conseqüência disso, podemos determinar uma região do espaço de $M^{2}$ no qual, tanto as contribuições de ordem mais altas na OPE quanto as dos estados excitados ou ressonantes são suprimidas, de tal maneira que os parâmetros fenomenológicos associados com o estado fundamental do hádron podem ser determinados. Portanto, devemos determinar um intervalo de $M^{2}$ onde essa comparação seja adequada, possibilitando a determinação de resultados confiáveis. Tal intervalo no espaço da massa de Borel é chamaddo de Janela de Borel.

\subsection{A Janela de Borel}

Para determinarmos o intervalo associado à Janela de Borel, devemos encontrar o valor máximo $M_{\max }$ e mínimo $M_{\min }$ da massa de Borel. A seguir, vamos apresentar alguns critérios que nos permitem determiná-los.

\subsubsection{Determinando o Máximo da Massa de Borel}

O objetivo das Regras de Soma da QCD é extrair informações a respeito do estado fundamental dos hádrons. Deste modo, estamos interessados no primeiro termo de (2.77), que será rotulado pela letra $P$ (Pólo). O segundo termo associado com o contínuo de ressonâncias, será definido como $C$ (Contínuo), assim, de (2.77), temos

$$
\begin{gathered}
P=\lambda^{2} e^{-m^{2} / M^{2}} ; \\
C=\int_{s_{0}}^{\infty} d s \rho^{O P E}(s) e^{-s / M^{2}} .
\end{gathered}
$$

Analisando (2.78) e (2.79), percebemos que à medida que a Massa de Borel cresce, a contribuição de $C$ aumenta enquanto $P$ diminui. Assim, deve existir um valor no qual as duas contribuições se igualam e é este valor de $M$ que será o limite superior da Janela de Borel, isto é, $M_{\max }$. Tal ponto pode ser observado na Figura 2.4 [20] que mostra as contribuições do pólo e do contínuo calculados usando as regras de soma para o estado X(4350) supondo que este seja um estado molecular $D_{s}^{*} D_{s 0}^{*}$. 
Este critério de determinação de $M_{\max }$ é chamado de Dominância do Pólo sobre o Contínuo.

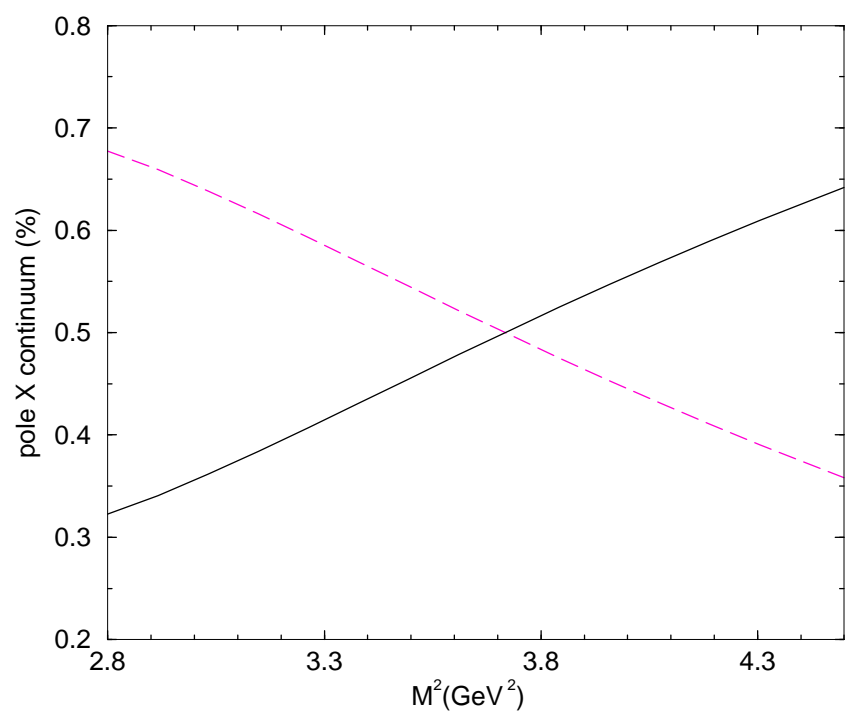

Figura 2.4: A linha contínua representa a variação da contribuição do Pólo $(P)$ com a Massa de Borel ao quadrado para $\sqrt{s_{0}}=5.3 \mathrm{GeV}$, enquanto a linha pontilhada representa a contribuição do contínuo $C$. Note que as contribuições se igualam quando $M^{2}$ for igual a $3.74 \mathrm{GeV}^{2}$, portanto, esse valor, para este caso, será o valor para $M_{\max }$.

\subsubsection{Determinando o Mínimo da Massa de Borel}

Pelo Lado da OPE, o uso da Transformada de Borel mapeia os coeficientes $\left(1 / Q^{2}\right)^{k}$ em $\left(1 / M^{2}\right)^{k}$, portanto, para grandes valores da Massa de Borel, os termos de ordem mais alta tornam-se menos importantes, melhorando a convergência da OPE. Podemos então determinar um valor mínimo abaixo do qual a série apresenta problemas de convergência. A Figura 2.5 [20] apresenta o comportamento da OPE à medida que adicionamos termos de ordem mais alta. Cada termo está dividido pela soma total de todas as contribuições dos termos considerados na série até dimensão 8, calculados para o caso do estado $X(4350)$. Observe que ao passo em que adicionamos mais termos, a curva associada se aproxima da reta assíntota cortando 
o eixo vertical no ponto de valor igual a 1 . Podemos notar que para valores abaixo de $3.2 \mathrm{GeV}^{2}$ na Massa de Borel, a série apresenta problemas na sua convergência. Portanto, estabelecemos tal valor de $M^{2}$ como o limite inferior da Janela de Borel, ou seja, $M_{\text {min }}$. Portanto, se a condição $M_{m a x}^{2}>M_{m i n}^{2}$ for satisfeita, temos então, uma região - Janela de Borel - onde os resultados das Regras de Soma são confiáveis.

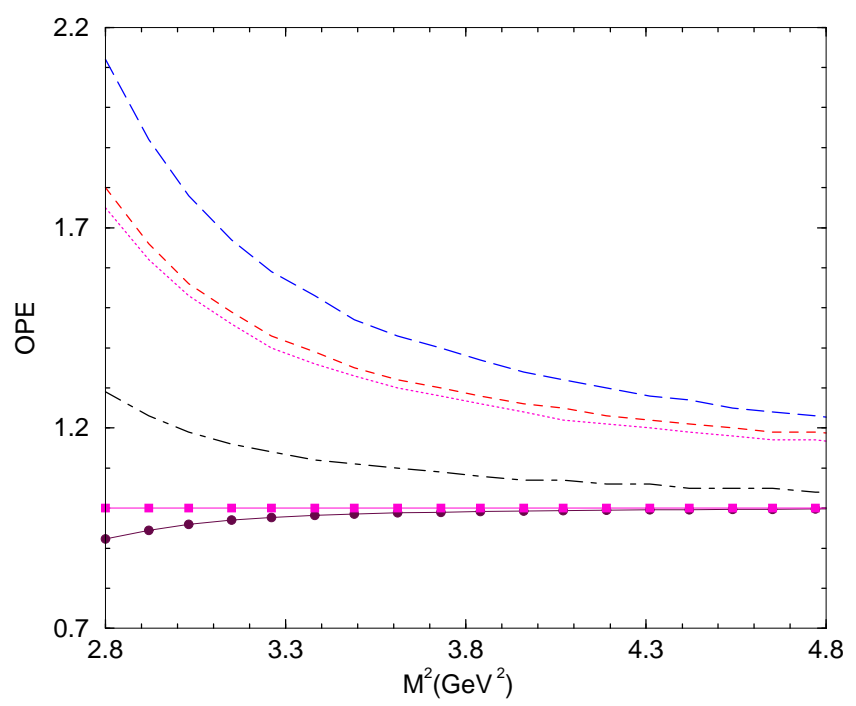

Figura 2.5: Comportamento da série devido ao acréscimo das contribuições relativas de cada um dos condensados. $s_{0}$, é o limiar do threshold para o qual o cálculo foi realizado $\left(\sqrt{s_{0}}=5.3 \mathrm{GeV}\right)$. Abaixo de $M^{2}$ igual a $3.2 \mathrm{GeV}^{2}$, é fácil ver que a série apresenta problemas de convergência.

\subsection{O Princípio da Dualidade Quark-Hádron e a Determinação dos Parâmetros Hadrônicos}

Uma vez calculada a função de correlação pelas duas abordagens, aplicamos a Transformada de Borel para suprimir no Lado Fenomenológico, a contribuição dos estados excitados e, no Lado da OPE, os termos de dimensão mais alta. Com isso, podemos, através do Princípio da Dualidade Quark-Hádron, comparar as descrições da função de correlação e, assim, obter os parâmetros fenomenológicos de interesse, 
como por exemplo, a massa do estado fundamental e a constante de acoplamento. Portanto, aplicando a transformada de Borel às equações (2.72), (2.70) e em seguida, igualando as duas, obtemos:

$$
\lambda^{2} e^{-m^{2} / M^{2}}+\int_{s_{0}}^{\infty} d s \rho^{O P E}(s) e^{-s / M^{2}}=\int_{s_{\min }}^{\infty} d s \rho^{O P E}(s) e^{-s / M^{2}} .
$$

Todavia, podemos separar o lado direito de (2.80) da seguinte maneira,

$$
\int_{s_{\min }}^{\infty} d s \rho^{O P E}(s) e^{-s / M^{2}}=\int_{s_{\min }}^{s_{0}} d s \rho^{O P E}(s) e^{-s / M^{2}}+\int_{s_{0}}^{\infty} d s \rho^{O P E}(s) e^{-s / M^{2}} .
$$

A finalidade disso, é que podemos cancelar os termos iguais em (2.80), isto é, as integrais com limites de 0 a infinito, obtendo deste modo,

$$
\lambda^{2} e^{-m^{2} / M^{2}}=\int_{s_{\min }}^{s_{0}} d s \rho^{O P E}(s) e^{-s / M^{2}} .
$$

Derivando (2.82) com relação a $1 / M^{2}$, resulta em

$$
m^{2} \lambda^{2} e^{-m^{2} / M^{2}}=\int_{s_{\min }}^{s_{0}} d s s \rho^{O P E}(s) e^{-s / M^{2}} .
$$

Assim, para obtermos a massa do estado estado fundamental dividimos (2.83) por (2.82), $\log 0$

$$
m^{2}=\frac{\int_{s_{\min }}^{s_{0}} d s s \rho^{O P E}(s) e^{-s / M^{2}}}{\int_{s_{\min }}^{s_{0}} d s \rho^{O P E}(s) e^{-s / M^{2}}} .
$$

É através desse resultado que calculamos a massa usando as Regras de Soma da QCD. É esta equação que usaremos para calcular as massas dos estados estudados nesse trabalho. 



\section{Capítulo 3}

\section{O Estado X(4350)}

\subsection{Os Números Quânticos e a Corrente}

A escolha da corrente interpolante desempenha um papel importante em Regras de Soma da QCD, pois nela devem estar contidas as informações a respeito dos números quânticos da ressonância a ser estudada. Além disso, a escolha da corrente envolve a hipótese acerca da estrutura interna da partícula e é esta suposição que será avaliada pelas Regras de Soma. Portanto, baseando-se nos números quânticos da estrutura estreita $X(4350)$ apresentaremos uma corrente que incorpora os números quânticos $J^{P C}$, onde $J, P$ e $C$, são respectivamente, o momento total, paridade e conjugação de carga.

Os números quânticos possíveis para um estado que decai em $J / \psi$ e $\phi$ são $J^{P C}=$ $0^{++}, J^{P C}=1^{-+}$e $J^{P C}=2^{++}$. Destes conjuntos de números quânticos, somente $1^{-+}$não está em concordância com o modelo de quarks, sendo portanto, considerado exótico. Na referência [29], os autores encontraram resultados consistentes com a massa do estado $X(4350)$ considerando-o um estado de tetraquarks com o conjunto de números quânticos $2^{++}$. Por outro lado, resultados consistentes com a previsão do $X(4350)$ como um estado molecular $D_{s}^{*+} \bar{D}_{s 0}^{*-}$, foram obtidos na referência [42] com $J^{P}=1^{-}$, mas, sem nenhum sinal de conjugação de carga definido.

Estudos considerando um estado molecular envolvendo um méson escalar e um 
méson vetorial [11], com conjugação de carga negativa, fornecem uma massa consistente com a do $X(4350)$. Contudo, os números quânticos não são consistentes com os números possíveis para o $X(4350)$ mencionados anteriormente.

Os mésons " $D$ " são formados por um par $c \bar{q}$. Dependendo do spin e do sinal da paridade, os mésons $D$ podem ser classificados de acordo com a tabela abaixo.

Tabela 3.1: Mésons $D$ associados com as respectivas correntes de acordo com o sinal da paridade e o valor do spin

\begin{tabular}{ccccc} 
Símbolo & Spin/Paridade & Nome & Corrente \\
\hline r & & & & \\
$D$ & $J^{P}=0^{-}$ & Pseudoescalar & $i \bar{c} \gamma_{5} q$ \\
$D^{*}$ & $J^{P}=1^{-}$ & Vetorial & $\bar{c} \gamma_{\mu} q$ \\
$D_{0}$ & $J^{P}=0^{+}$ & Escalar & $\bar{c} q$ \\
$D_{1}$ & $J^{P}=1^{+}$ & Axial & $\bar{c} \gamma_{5} \gamma_{\mu} q$
\end{tabular}

Portanto, conhecidos os números quânticos (paridade, spin e conjugação de carga), podemos associar uma corrente para uma possível descrição do estado molecular $X(4350)$ em termos dos mésons $D$.

Como discutido acima, o $X(4350)$ decai em $J / \psi \phi$, isso fornece uma conjugação de carga positiva para esse estado. Da adição de momento angular, temos $J_{\max }=$ $\left|J_{1}+J_{2}\right|$ e $J_{\min }=\left|J_{1}-J_{2}\right|$. Como $J_{1}=1$ é o momento angular do $J / \psi$ e $J_{2}=1$ o momento angular do $\phi$, o momento angular total do estado $X(4350)$ será 0,1 ou 2. Para um estado decaindo num par de mésons, sabemos que a paridade é dada por:

$$
P=(-1)^{l} P_{1} P_{2},
$$

onde $l$ é o momento angular orbital do par e $P_{1}$ e $P_{2}$ são as paridades de cada um dos mésons no par. Assim, em onda $S(l=0)$, os seguintes valores para o momento 
angular total e paridade, são possíveis para o estado $X(4350)$ :

$$
J^{P}=\left\{\begin{array}{l}
0^{+} \\
1^{+} \\
2^{+}
\end{array}\right.
$$

Em termos do momento angular orbital e do spin $(s)$ do estado, a conjugação de carga é dada por:

$$
C=(-1)^{l+s}
$$

Deste modo, usando (3.1) e (3.2) obtemos:

$$
J^{P C}=\left\{\begin{array}{l}
0^{++} \\
1^{+-} \\
2^{++}
\end{array}\right.
$$

Assim vemos que os números quânticos $J=1, l=0$ e $s=1$ não são possíveis para o $X(4350)$, já que este tem $C=+$. Por outro lado, o estado com $J=1$ pode ser construído com os mésons $J / \psi$ e $\phi$ em onda $P(l=1)$ e $s=1$. Com isso conseguimos $J^{P C}=1^{-+}$. Portanto, os números quânticos possíveis para o $X(4350)$ são:

$$
J^{P C}=\left\{\begin{array}{c}
0^{++} \\
1^{-+} \\
2^{++}
\end{array}\right.
$$

Por outro lado, para um par quark-antiquark, temos que $P=-(-1)^{l}$, e portanto os números quânticos possíveis para um par quark-antiquark com momento angular total igual a 1 são:

$$
q \bar{q} \Longrightarrow\left\{\begin{array}{r}
l=1, s=0 \Rightarrow J^{P C}=1^{+-} \\
l=1, s=1 \Rightarrow J^{P C}=0^{++}, 1^{++}, 2^{++} \\
l=0, s=1 \Rightarrow J^{P C}=1^{--}
\end{array}\right.
$$

da onde vemos que não é possível o conjunto de números quânticos $1^{-+}$, para um par $q \bar{q}$. Portanto, o estado descrito pelos números quânticos $J^{P C}=1^{-+}$é necessariamente um estado exótico. 
Apoiando-se no exposto acima, vamos investigar se o $X(4350)$ pode ser descrito como uma molécula $D_{s o} \bar{D}_{s}^{*}$, com números quânticos $J^{P C}=1^{-+}$.

\subsubsection{Conjugação de Carga e a Paridade da Corrente}

Usando as correntes dadas na Tabela 3.1 para mésons $D$, podemos construir uma corrente mesônica que pode ser um auto-estado do operador de conjugação de carga "C" e paridade " $P$ ". Uma possível corrente pode ser a combinação

$$
j_{\mu}=\frac{1}{\sqrt{2}}\left[\left(\bar{s}_{a} \gamma_{\mu} c_{a}\right)\left(\bar{c}_{b} s_{b}\right)-\left(\bar{c}_{d} \gamma_{\mu} s_{d}\right)\left(\bar{s}_{e} c_{e}\right)\right]
$$

onde $a, b, d$ e $e$, são índices de cor, $s$ e $c$ são os operadores de campo do quark strange e charm, respectivamente. O próximo passo é verificar se a corrente (3.3) possui os números quânticos consistentes com $J^{P C}=1^{-+}$. Sejam $(\psi)_{c}$ e $(\psi)_{p}$, os campos fermiônicos dos quarks transformados por conjugação de carga e paridade, respectivamente. Portanto, temos as seguintes relações [25]:

$$
\begin{gathered}
(\bar{\psi})_{c}=-\psi^{T} C^{-1} \\
(\psi)_{c}=C \bar{\psi}^{T} \\
(\psi)_{p}=\gamma_{0} \psi \\
(\bar{\psi})_{c}=\bar{\psi} \gamma_{0} .
\end{gathered}
$$

Sendo assim, podemos determinar o sinal da conjugação de carga da corrente (3.3) usando as equações (3.4) e (3.5). Deste modo,

$$
\begin{aligned}
\left(j_{\mu}\right)_{c} & =\frac{1}{\sqrt{2}}\left[\left(-s_{a}^{T} C^{-1} \gamma_{\mu} C \bar{c}_{a}^{T}\right)\left(-c_{b}^{T} C^{-1} C \bar{s}_{b}^{T}\right)-\right. \\
& \left.-\left(-c_{d}^{T} C^{-1} \gamma_{\mu} C \bar{s}_{d}^{T}\right)\left(-s_{e}^{T} C^{-1} C \bar{c}_{e}^{T}\right)\right] .
\end{aligned}
$$

Por outro lado usando $C^{-1} \gamma_{\mu} C=-\gamma_{\mu}^{T}$ e $C^{-1} C=\hat{1}$ [31]. Reorganizando os termos, obtemos

$$
\left(j_{\mu}\right)_{c}=\frac{1}{\sqrt{2}}\left[\left(\bar{s}_{a} \gamma_{\mu} \bar{s}_{a}\right)\left(\bar{c}_{b} s_{b}\right)-\left(\bar{c}_{d} \gamma_{\mu} s_{d}\right)\left(\bar{s}_{e} c_{e}\right)\right] .
$$


Portanto, a corrente (3.3) possui sinal + por transformação de conjugação de carga, isto é, $\left(j_{\mu}\right)_{c}=+j_{\mu}$. Para determinarmos o sinal da paridade da corrente (3.3), usamos (3.6) e (3.7), deste modo, segue que

$$
\left(j_{\mu}\right)_{p}=\frac{1}{\sqrt{2}}\left[\left(\bar{s}_{a} \gamma_{0} \gamma_{\mu} \gamma_{0} c_{a}\right)\left(\bar{c}_{b} \gamma_{0} \gamma_{0} s_{b}\right)-\left(\bar{c}_{a} \gamma_{0} \gamma_{\mu} \gamma_{0} s_{a}\right)\left(\bar{s}_{b} \gamma_{0} \gamma_{0} c_{b}\right)\right]
$$

Por outro lado, das relações de anticomutação entre as matrizes de Dirac, segue que $\left\{\gamma_{0}, \gamma_{\mu}\right\}=0$, assim, $\gamma_{0} \gamma_{\mu}=-\gamma_{\mu} \gamma_{0}$, portanto,

$$
\begin{aligned}
\left(j_{\mu}\right)_{p} & =\frac{1}{\sqrt{2}}\left[\left(-\bar{s}_{a} \gamma_{\mu} \gamma_{0} \gamma_{0} c_{a}\right)\left(\bar{c}_{b} \gamma_{0} \gamma_{0} s_{b}\right)-\left(-\bar{c}_{a} \gamma_{\mu} \gamma_{0} \gamma_{0} s_{a}\right)\left(\bar{s}_{b} \gamma_{0} \gamma_{0} c_{b}\right)\right] \\
& =\frac{1}{\sqrt{2}}\left[\left(-\bar{s}_{a} \gamma_{\mu} \gamma_{0}^{2} c_{a}\right)\left(\bar{c}_{b} \gamma_{0}^{2} s_{b}\right)-\left(-\bar{c}_{a} \gamma_{\mu} \gamma_{0}^{2} s_{a}\right)\left(\bar{s}_{b} \gamma_{0}^{2} c_{b}\right)\right] \\
& =-\frac{1}{\sqrt{2}}\left[\left(\bar{s}_{a} \gamma_{\mu} c_{a}\right)\left(\bar{c}_{b} s_{b}\right)-\left(\bar{c}_{a} \gamma_{\mu} s_{a}\right)\left(\bar{s}_{b} c_{b}\right)\right] \\
& =-j_{\mu} .
\end{aligned}
$$

Deste modo, a corrente (3.3) tem paridade ímpar. Com isso, podemos constatar que a corrente escolhida possui os números quânticos propostos.

\subsection{Lado da OPE}

Escolhida a corrente consistente com os números quânticos escolhidos, iniciamos o cálculo do Lado da OPE. substituindo a corrente (3.3) na função de correlação de dois pontos dada pela Equação (2.1). Como a corrente (3.3) não é conservada, podemos reescrever (2.1) em termos de duas estruturas de Lorentz independentes. Deste modo, temos

$$
\Pi_{\mu \nu}(q)=-\Pi_{1}\left(q^{2}\right)\left(g_{\mu \nu}-\frac{q_{\mu} q_{\nu}}{q^{2}}\right)+\Pi_{0}\left(q^{2}\right) \frac{q_{\mu} q_{\nu}}{q^{2}} .
$$

As funções $\Pi_{1}\left(q^{2}\right)$ e $\Pi_{0}\left(q^{2}\right)$ possuem números quânticos de mésons de spin 1 e 0 respectivamente. Como estamos interessados em estudar uma partícula de spin 1 , devemos trabalhar com a função $\Pi_{1}\left(q^{2}\right)$ isoladamente pois, somente ela dará contribuições para um estado com números quânticos $1^{-+}$. Para isso escolhemos $g_{\mu \nu}$. 
Inserindo a corrente (3.3) no correlator (2.1) definido no Capítulo 2, obtemos a seguinte expressão

$$
\begin{aligned}
& \Pi(q)=i \int d^{4} x e^{i q x} \frac{1}{2}\left[\operatorname{Tr}\left\{S_{g a}^{s}(-x) \gamma_{\mu} S_{a g}^{c}(x) \gamma_{\nu}\right\} \operatorname{Tr}\left\{S_{b f}^{s}(x) S_{f b}^{c}(-x)\right\}+\right. \\
& -\operatorname{Tr}\left\{S_{a h}^{c}(x) S_{h a}^{s}(-x) \gamma_{\mu}\right\} \operatorname{Tr}\left\{S_{b w}^{s}(x) \gamma_{\nu} S_{w b}^{c}(-x)\right\}-\operatorname{Tr}\left\{S_{f d}^{c}(-x) \gamma_{\mu} S_{d f}^{s}(x)\right\} \times \\
& \left.\times \operatorname{Tr}\left\{S_{e g}^{c}(x) \gamma_{\nu} S_{g e}^{s}(-x)\right\}+\operatorname{Tr}\left\{S_{w d}^{c}(-x) \gamma_{\mu} S_{d w}^{s}(x) \gamma_{\nu}\right\} \operatorname{Tr}\left\{S_{e h}^{c}(x) S_{h e}^{s}(-x)\right\}\right],
\end{aligned}
$$

onde $S^{c}$ e $S^{s}$ são os propagadores dos quarks charm e strange, respectivamente. Note que a presença do sinal negativo no argumento de alguns dos propagadores da Equação (3.13), está associado com a idéia de que os anti-quarks podem ser representados como quarks propagando-se para trás no espaço-tempo. Por exemplo, $S^{c}(-x)$, representa o propagador do anti-quark charm enquanto $S^{s}(-x)$ representa o propagador do anti-quark strange.

Para quarks pesados usamos a expressão do propagador no espaço dos momentos. O propagador associado ao quark charm no espaço dos momentos é escrito da seguinte maneira:

$$
S^{c}(p)=\int \frac{d^{4} p}{(2 \pi)^{4}} e^{-i p x} S^{c}(x)
$$

Substituindo (3.14) na equação (3.13), obtemos

$$
\begin{aligned}
& \Pi(q)=i \int \frac{d^{4} x d^{4} p_{1} d^{4} p_{2}}{(2 \pi)^{8}} e^{i q x} e^{-i p_{1} x} e^{-i p_{2} x} \frac{1}{2}\left[\operatorname{Tr}\left\{S_{g a}^{s}(-x) \gamma_{\mu} S_{a g}^{c}(p) \gamma_{\nu}\right\} \operatorname{Tr}\left\{S_{b f}^{s}(x) S_{f b}^{c}(-p)\right\}\right. \\
& \quad-\operatorname{Tr}\left\{S_{a h}^{c}(p) S_{h a}^{s}(-x) \gamma_{\mu}\right\} \operatorname{Tr}\left\{S_{b w}^{s}(x) \gamma_{\nu} S_{w b}^{c}(-p)\right\}-\operatorname{Tr}\left\{S_{f d}^{c}(-p) \gamma_{\mu} S_{d f}^{s}(x)\right\} \times \\
& \left.\quad \times \operatorname{Tr}\left\{S_{e g}^{c}(p) \gamma_{\nu} S_{g e}^{s}(-x)\right\}+\operatorname{Tr}\left\{S_{w d}^{c}(-p) \gamma_{\mu} S_{d w}^{s}(x) \gamma_{\nu}\right\} \operatorname{Tr}\left\{S_{e h}^{c}(p) S_{h e}^{s}(-x)\right\}\right]
\end{aligned}
$$

Antes de calcularmos as contribuições da Equação (3.15) vamos, nas próximas seções, definir todos os diagramas que contribuem para o Lado da OPE. Em seguida, substituiremos as expressões dos propagadores dos quarks de acordo com a construção de cada diagrama. Por definição, as linhas superiores (linhas cheias) dos diagramas representam um méson enquanto as duas inferiores (linhas pontilhadas) 
representam o outro méson como mostra a Figura 3.1.

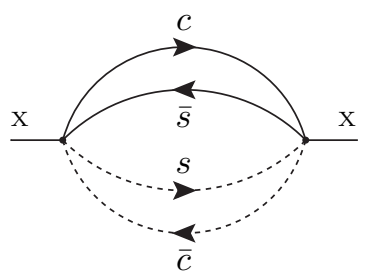

Figura 3.1: Diagrama representando um estado molecular formado por dois mésons, as duas linhas superiores representam um méson formado por um quark $c$ e um anti-quark $s$ enquanto as duas linhas inferiores representam um méson formado por um quark $s$ e um anti-quark $c$.

Uma lista completa dos propagadores a serem utilizados no cálculo das contribuições de cada diagrama, podem ser encontrados nas referências [24] e [32]. Além disso, no Apêndice A, listamos todos os propagadores utilizados neste trabalho.

Na Regra de Soma para o X(4350) consideramos todas as contribuições mencionadas na Seção 2.2 do Capítulo 2, isto é, contribuições perturbativas, não-fatorizáveis e não-perturbativas até dimensão 8. Calculamos os termos dominantes para cada dimensão da OPE, mantendo os termos lineares na massa do quark $s$ e na massa do quark c. Além disso, determinamos as densidades espectrais associadas com cada dimensão da OPE. Desta maneira a densidade espectral total presente na Equação (2.84) será dada por

$$
\begin{aligned}
\rho^{O P E}(s) & =\rho^{\text {pert }}(s)+\rho^{\langle\bar{q} q\rangle}(s)+\rho^{\left\langle G^{2}\right\rangle}(s)+\rho_{1}^{\text {mix }}(s)+ \\
& +\rho_{2}^{\text {mix }}(s)+\rho^{\langle\bar{q} q\rangle^{2}}(s)+\rho^{\text {mix }\langle\bar{q} q\rangle}(s) .
\end{aligned}
$$

\subsubsection{Diagramas Perturbativos}

Os diagramas perturbativos que contribuem para a OPE estão mostrados na Figura 3.2 


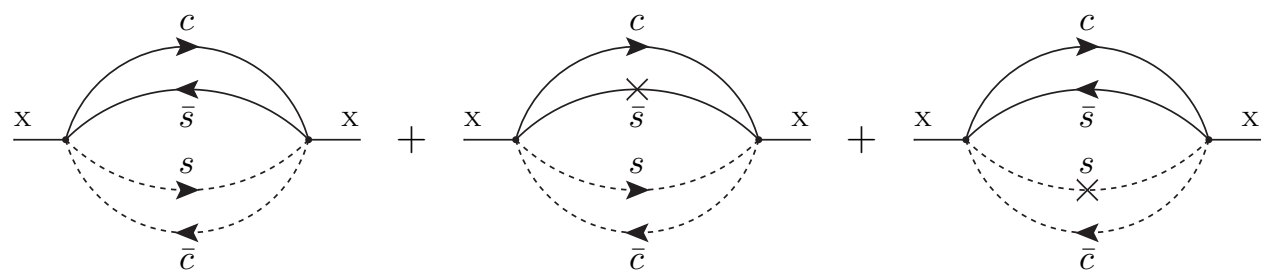

Figura 3.2: Diagramas perturbativos. Note que, o símbolo $\times$ presente nas linhas do quark e do anti-quark s, representam a correção na massa dos mesmos.

Esses diagramas envolvem somente propagadores de quarks livres. Para o primeiro diagrama temos:

$$
\begin{gathered}
S_{a b}^{c}(p)=\frac{i\left(\not p+m_{c}\right) \delta_{a b}}{p^{2}-m^{2}} ; \\
S_{a b}^{s}(x)=\frac{i \not x \delta_{a b}}{2 \pi^{2} x^{4}} .
\end{gathered}
$$

Substituindo os propagadores acima na Equação (3.15), obtemos

$$
\begin{aligned}
& \Pi_{\mu \nu}=\frac{i}{2} \int d^{4} x d^{4} p_{1} d^{4} p_{2} \frac{e^{i q x-i\left(p_{1}+p_{2}\right) x}}{(2 \pi)^{8}}\left[\operatorname{Tr}\left\{\frac{-i \not x}{2 \pi^{2} x^{4}} \gamma_{\mu} \frac{i\left(\not p_{1}+m_{c}\right)}{p_{1}^{2}-m_{c}^{2}} \gamma_{\nu}\right\} \times\right. \\
& \times \operatorname{Tr}\left\{\frac{i \not x}{2 \pi^{2} x^{4}}(-i) \frac{\left(\not \gamma_{2}-m_{c}\right)}{p_{2}^{2}-m_{c}^{2}}\right\} \delta_{g a} \delta_{a g} \delta_{b f} \delta_{f b} \\
& -\operatorname{Tr}\left\{\frac{i\left(\not{ }_{1}+m_{c}\right)}{p_{1}^{2}-m_{c}^{2}}(-i) \frac{\not x}{2 \pi^{2} x^{4}} \gamma_{\mu}\right\} \operatorname{Tr}\left\{\frac{i \not x}{2 \pi^{2} x^{4}} \gamma_{\nu}(-i) \not{ }_{2}^{2}-m_{c}^{2}\right\} \delta_{a h} \delta_{h a} \delta_{b w} \delta_{w b} \\
& -\operatorname{Tr}\left\{(-i) \frac{\left(\not \phi_{2}-m_{c}\right)}{p_{2}^{2}-m_{c}^{2}} \gamma_{\mu} \frac{i \not \not}{2 \pi^{2} x^{4}}\right\} \operatorname{Tr}\left\{\frac{\left.i(\not)_{1}+m_{c}\right)}{p_{1}^{2}-m_{c}^{2}} \gamma_{\nu}(-i) \frac{\not x}{2 \pi^{2} x^{4}}\right\} \delta_{f d} \delta_{d f} \delta_{e g} \delta_{g e} \\
& \left.+\operatorname{Tr}\left\{(-i) \frac{\left(\not d_{2}-m_{c}\right)}{p_{2}^{2}-m_{c}^{2}} \gamma_{\mu} \frac{i \not x}{2 \pi^{2} x^{4}} \gamma_{\nu}\right\} \operatorname{Tr}\left\{\frac{i\left(\not d_{1}+m_{c}\right)}{p_{1}^{2}-m_{c}^{2}}(-i) \frac{\not x}{2 \pi^{2} x^{4}}\right\} \delta_{w d} \delta_{d w} \delta_{e h} \delta_{h e}\right] .
\end{aligned}
$$

Por outro lado, $\sum \delta_{a i} \delta_{i a} \delta_{b j} \delta_{j b}=9$ [25], deste modo, a equação acima pode ser reescrita como

$$
\begin{aligned}
\Pi_{\mu \nu} & =\frac{9 i}{2^{10} \pi^{12}} \int d^{4} x d^{4} p_{1} d^{4} p_{2} \frac{e^{i q x-i\left(p_{1}+p_{2}\right) x}}{x^{8}\left(p_{1}^{2}-m_{c}^{2}\right)\left(p_{2}^{2}-m_{c}^{2}\right)}\left[\operatorname{Tr}\left\{\not x \gamma_{\mu}\left(\left.\not\right|_{1}+m_{c}\right) \gamma_{\nu}\right\} \times\right. \\
& \times \operatorname{Tr}\left\{\not x\left(\left.\not\right|_{2}-m_{c}\right)\right\}-\operatorname{Tr}\left\{\left(\not \phi_{1}+m_{c}\right) \not x \gamma_{\mu}\right\} \operatorname{Tr}\left\{i \not x \gamma_{\nu}\left(\not \phi_{2}^{2}-m_{c}^{2}\right)\right\}-
\end{aligned}
$$




$$
\begin{aligned}
& -\operatorname{Tr}\left\{\left(\not \phi_{2}-m_{c}\right) \gamma_{\mu} \not\right\} \operatorname{Tr}\left\{\left(\not \phi_{1}+m_{c}\right) \gamma_{\nu} \not x+\right. \\
& \left.+\operatorname{Tr}\left\{\left(\not \phi_{2}-m_{c}\right) \gamma_{\mu} \not \gamma_{\nu}\right\} \operatorname{Tr}\left\{\left(\not \phi_{1}+m_{c}\right) \not\right\}\right] .
\end{aligned}
$$

Calculando os traços (Ver Apêndice X) obtemos:

$$
\begin{gathered}
\Pi_{\mu \nu}=\frac{9 i}{2^{7} \pi^{12}} \int d^{4} x d^{4} p_{1} d^{4} p_{2} \frac{e^{i q x-i\left(p_{1}+p_{2}\right) x}}{x^{8}\left(p_{1}^{2}-m_{c}^{2}\right)\left(p_{2}^{2}-m_{c}^{2}\right)}\left[m_{c}^{2} x_{\mu} x_{\nu}-\right. \\
\left.-\left(x \cdot p_{1}\right)\left(x \cdot p_{2}\right) g_{\mu \nu}+\left(x \cdot p_{2}\right)\left(p_{1 \nu} x_{\mu}+p_{1 \mu} x_{\nu}\right)\right] .
\end{gathered}
$$

As contribuições dos dois últimos diagramas da Figura 3.2, são obtidas acrescentando a expressão do propagador que representa a correção de massa do quark s dada pela equação abaixo

$$
S^{s}(x)=-\frac{m_{s} \delta_{a b}}{4 \pi^{2} x^{2}}
$$

Portanto, de forma análoga ao cálculo do diagrama anterior, substituímos as expressões dos propagadores na Equação (3.15), obtendo

$$
\begin{aligned}
& \Pi_{\mu \nu}^{m_{s}}=2 \frac{-9}{(2 \pi)^{12}} \int d^{4} x d^{4} p_{1} d^{4} p_{2} \frac{e^{i q x-i\left(p_{1}+p_{2}\right) x}}{x^{6}\left(p_{1}^{2}-m_{c}^{2}\right)\left(p_{2}^{2}-m_{c}^{2}\right)}\left[\operatorname{Tr}\left\{-m_{s} \gamma_{\mu}(\not)_{1}+m_{c}\right) \gamma_{\nu}\right\} \times \\
& \times \operatorname{Tr}\left\{\not x\left(\not \phi_{2}-m_{c}\right)\right\}-\operatorname{Tr}\left\{\left(\not \phi_{1}+m_{c}\right)\left(-m_{s}\right) \not x \gamma_{\mu}\right\} \operatorname{Tr}\left\{\not x \gamma_{\nu}\left(\not \phi_{2}^{2}-m_{c}^{2}\right)\right\} \\
& -\operatorname{Tr}\left\{\left(\not \phi_{2}-m_{c}\right) \gamma_{\mu} \not\right\} \operatorname{Tr}\left\{\left(\not \phi_{1}+m_{c}\right) \gamma_{\nu}\left(-m_{s}\right)\right\} \\
& \left.+\operatorname{Tr}\left\{\left(\not \gamma_{2}-m_{c}\right) \gamma_{\mu} \not c \gamma_{\nu}\right\} \operatorname{Tr}\left\{\left(\not \phi_{1}+m_{c}\right)\left(-m_{s}\right)\right\}\right] \text {. }
\end{aligned}
$$

Novamente calculando os traços, temos:

$\Pi_{\mu \nu}^{m_{s}}=\frac{9 m_{s} m_{c}}{2^{8} \pi^{12}} \int d^{4} x d^{4} p_{1} d^{4} p_{2} \frac{e^{i q x-i\left(p_{1}+p_{2}\right) x}}{x^{6}\left(p_{1}^{2}-m_{c}^{2}\right)\left(p_{2}^{2}-m_{c}^{2}\right)}\left[x_{\nu}\left(p_{1 \mu}+p_{2 \mu}\right)+x_{\mu}\left(p_{1 \nu}+p_{2 \nu}\right)\right]$.

O próximo passo é calcular as integrais (3.20) e (3.22) e em seguida, escrever o resultado na forma de uma relação de dispersão. Os detalhes do cálculo das integrais de todas as contribuições do Lado da OPE, são dados no Apêndice X. Assim, para as Equações (3.20) e (3.22), obtemos

$$
\begin{aligned}
\Pi^{p e r t}\left(q^{2}\right) & =-\frac{1}{2^{12} \pi^{6}} \int_{0}^{1} \frac{d \alpha \beta}{\alpha^{3} \beta^{3}} \theta(1-\alpha-\beta)(1-\alpha-\beta) E^{3}(\alpha, \beta)\left[2 m_{c}^{2}(1-\alpha-\beta)^{2}+\right. \\
& +3 E(\alpha, \beta)(1+\alpha+\beta)] \ln F(\alpha, \beta)
\end{aligned}
$$




$$
\Pi^{m s . p e r t}\left(q^{2}\right)=-\frac{3 m_{c} m_{s}}{2^{9} \pi^{6}} \int_{0}^{1} \frac{d \alpha \beta}{\alpha^{3} \beta^{2}} \theta(1-\alpha-\beta)(1-\alpha-\beta)^{2} F^{3}(\alpha, \beta) \ln F(\alpha, \beta) .
$$

onde $F(\alpha, \beta)=m_{c}^{2}(\alpha+\beta)-\alpha \beta q^{2}$.

Usando a relação $\rho(s)=\frac{1}{\pi} \operatorname{Im}[\Pi(s)]$ [24], onde $s=q^{2}$, determinamos respectivamente, as contribuições sem e com correção de massa para a densidade espectral associada à contribuição perturbativa:

$$
\begin{gathered}
\rho^{\text {pert }}(s)=-\frac{1}{2^{12} \pi^{6}} \int_{\alpha_{\min }}^{\alpha_{\max }} \frac{d \alpha}{\alpha^{3}} \int_{\beta_{\min }}^{1-\alpha} \frac{d \beta}{\beta^{3}}(1-\alpha-\beta) F^{3}(\alpha, \beta)\left[2 m_{c}^{2}(1-\alpha-\beta)^{2}+\right. \\
+3 F(\alpha, \beta)(1+\alpha+\beta)] \ln F(\alpha, \beta) \\
\rho^{m_{s} \cdot \text { pert }}(s)=\frac{3 m_{c} m_{s}}{2^{9} \pi^{6}} \int_{\alpha_{\min }}^{\alpha_{\max }} \frac{d \alpha}{\alpha^{3}} \int_{\beta_{\min }}^{1-\alpha} \frac{d \beta}{\beta^{2}}(1-\alpha-\beta)^{2} F^{3}(\alpha, \beta)
\end{gathered}
$$

Os limites $\alpha_{\min }, \alpha_{\max }, \beta_{\min }$ e $1-\alpha$, são determinados da condição $F(\alpha, \beta)<0$ imposta sobre o termo no argumento do logaritmo para que a Equações (3.23) e (3.24) possam ter uma parte imaginária. Assim,

$$
m_{c}^{2}(\alpha+\beta)-\alpha \beta s<0
$$

Da função $\theta$ de Heaviside obtemos a condição $\alpha+\beta \leq 1$. Portanto, dessas duas condições temos os seguintes limites sobre os valores de $\alpha, \beta$ e $s$ :

$$
\begin{gathered}
\frac{1-\sqrt{1-4 m_{c}^{2} / s}}{2}<\alpha<\frac{1+\sqrt{1-4 m_{c}^{2} / s}}{2} ; \\
\frac{\alpha m_{c}^{2}}{\left(s \alpha-m_{c}^{2}\right)}<\beta<1-\alpha .
\end{gathered}
$$

Como $\alpha$ é real, o termo presente na raiz quadrada da desigualdade (3.28) deve ser positivo, deste modo, concluímos que $s>4 m_{c}^{2}$. Tais limites serão os mesmos para as demais densidades espectrais.

As contribuições dos demais diagramas associados a ordens superiores que contribuem para a OPE até dimensão 8 são calculadas de forma análoga: substituímos 
os propagadores associados com cada linha dos diagramas na Equação (3.15) e em seguida, calculamos os traços. Depois disso, calculamos as integrais e por fim escrevemos o resultado em termos de uma relação de dispersão. 


\subsubsection{Diagramas dos Condensados de Quarks}

Na Figura abaixo estão ilustrados os diagramas que contribuem para o condensado de quarks.
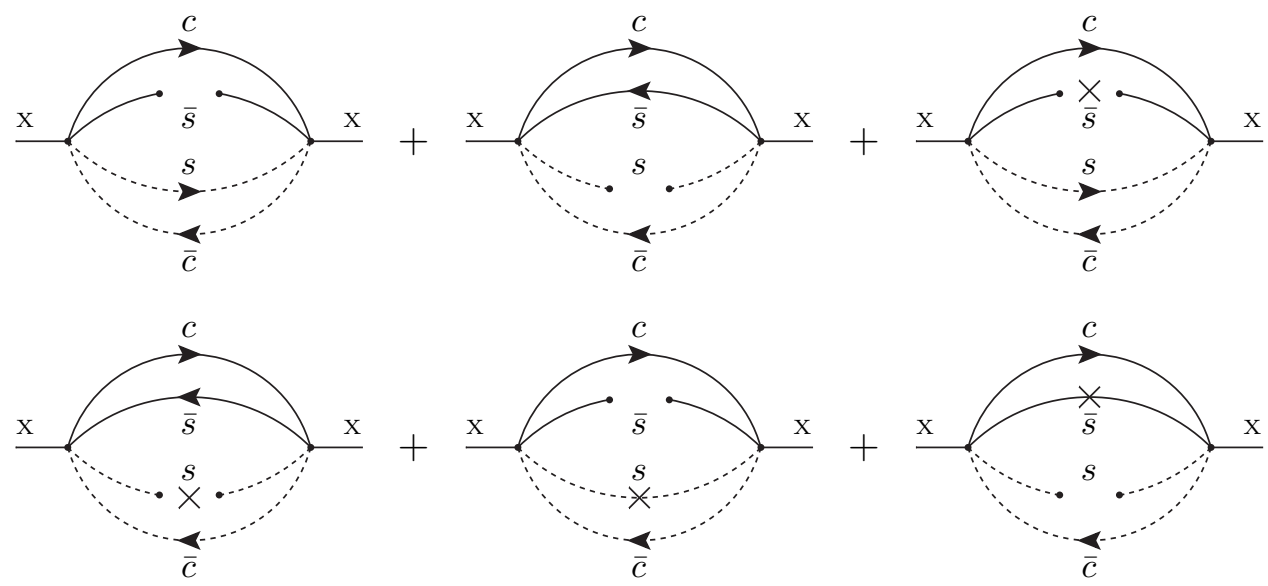

Figura 3.3: Diagramas associados aos Condensados de Quarks.

Para os dois primeiros diagramas, após substituirmos os propagadores e em seguida, calcularmos os traços, obtemos:

$\Pi_{\mu \nu}=\frac{3 m_{c}\langle\bar{s} s\rangle}{2^{7} \pi^{10}} \int d^{4} x d^{4} p_{1} d^{4} p_{2} \frac{e^{i q x-i\left(p_{1}+p_{2}\right) x}}{x^{4}\left(p_{1}^{2}-m_{c}^{2}\right)\left(p_{2}^{2}-m_{c}^{2}\right)}\left[x_{\mu}\left(p_{1 \nu}+p_{2 \nu}\right)+x_{\nu}\left(p_{1 \mu}+p_{2 \mu}\right)\right]$.

Calculando a integral acima e logo após isso, escrevendo o resultado como uma relação de dispersão, obtemos:

$$
\rho^{\langle\bar{s} s\rangle}(s)=-\frac{3 m_{c}\langle\bar{s} s\rangle}{2^{6} \pi^{4}} \int_{\alpha_{\min }}^{\alpha_{\max }} \frac{d \alpha}{\alpha^{2}} \int_{\beta_{\min }}^{1-\alpha} \frac{d \beta}{\beta}(1-\alpha-\beta) F^{2}(\alpha, \beta) .
$$

Os demais diagramas da Figura 3.3, representam a correção na massa do quark s. Por questão de simplicidade, vamos dividir suas contribuições em $\Pi_{A \mu \nu}^{m_{s}}$ e $\Pi_{B \mu \nu}^{m_{s}}$. Portanto, temos

$$
\Pi_{A \mu \nu}^{m_{s}}=-\frac{3 i m_{c}\langle\bar{s} s\rangle}{2^{7} \pi^{10}} \int d^{4} x d^{4} p_{1} d^{4} p_{2} \frac{e^{i q x-i\left(p_{1}+p_{2}\right) x}}{x^{2}\left(p_{1}^{2}-m_{c}^{2}\right)\left(p_{2}^{2}-m_{c}^{2}\right)}\left[m_{c}^{2} g_{\mu \nu}+p_{1 \mu} p_{2 \nu}\right],
$$




$$
\begin{gathered}
\Pi_{B \mu \nu}^{m_{s}}=\frac{3 i m_{c}\langle\bar{s} s\rangle}{2^{9} \pi^{10}} \int d^{4} x d^{4} p_{1} d^{4} p_{2} \frac{e^{i q x-i\left(p_{1}+p_{2}\right) x}}{x^{4}\left(p_{1}^{2}-m_{c}^{2}\right)\left(p_{2}^{2}-m_{c}^{2}\right)}\left[\left(x_{\nu} p_{2 \mu}+x_{\mu} p_{2 \nu}\right)\left(x \cdot p_{1}\right)+\right. \\
\left.+2 m_{c}^{2} x_{\mu} x_{\nu}+\left(x_{\nu} p_{1 \mu}+x_{\mu} p_{1 \nu}\right)\left(x \cdot p_{2}\right)-2 g_{\mu \nu}\left(x \cdot p_{1}\right)\left(x \cdot p_{2}\right)\right]
\end{gathered}
$$

Calculando as integrais (3.33) e (3.34) obtemos, após adicioná-las, a seguinte expressão para a densidade espectral:

$$
\begin{gathered}
\rho^{m_{s}\langle\bar{s} s\rangle}(s)=\frac{3 m_{s}\langle\bar{s} s\rangle}{2^{7} \pi^{4}}\left[\int_{\alpha_{\min }}^{\alpha_{\max }} \frac{d \alpha}{\alpha} \int_{\beta_{\min }}^{1-\alpha} \frac{d \beta}{\beta} m_{c}^{2}(3+\alpha+\beta) E(\alpha, \beta)\right. \\
\left.-\int_{\alpha_{\min }}^{\alpha_{\max }} \frac{d \alpha}{\alpha(1-\alpha)} H^{2}(\alpha)\right],
\end{gathered}
$$

onde $H(\alpha)$ é definido como $H(\alpha)=m_{c}^{2}-\alpha(1-\alpha) q^{2}$.

\subsubsection{Diagramas dos Condensados de Glúons}

Os diagramas que contribuem para o Condensado de Glúons podem ser vistos na Figura 3.4.
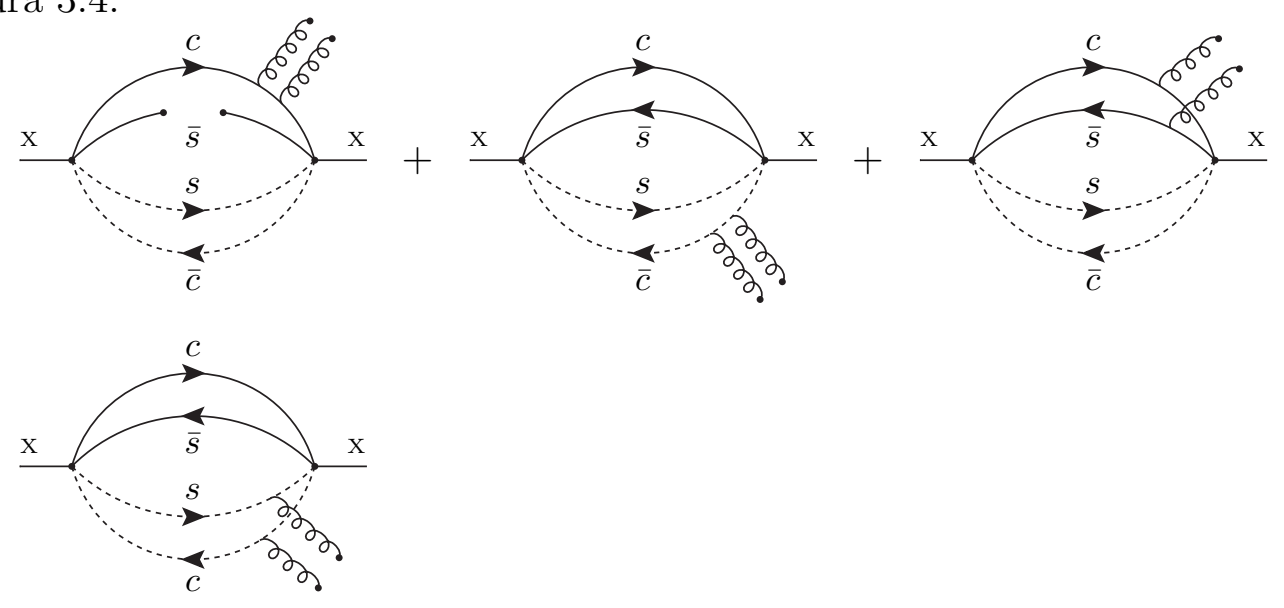

Figura 3.4: Diagramas associados aos Condensados de Glúons.

A contribuição para o Condensado de Glúons devido aos dois primeiros diagramas, após a substituição dos propagadores, será

$$
\Pi_{\mu \nu}^{(1)}(q)=\frac{3 i m_{c}\left\langle g_{s}^{2} G^{2}\right\rangle}{2^{8} \pi^{12}} \int d^{4} x d^{4} p_{1} d^{4} p_{2} \frac{e^{i q x-i\left(p_{1}+p_{2}\right) x}}{x^{8}\left(p_{1}^{2}-m_{c}^{2}\right)\left(p_{2}^{2}-m_{c}^{2}\right)^{4}}\left[\left(x_{\nu} p_{2 \mu}+x_{\mu} p_{2 \nu}\right)\left(x \cdot p_{1}\right)+\right.
$$




$$
-2 g_{\mu \nu}\left(x \cdot p_{1}\right)\left(x \cdot p_{2}\right)+\left(x_{\nu} p_{1 \mu}+x_{\mu} p_{1 \nu}\right)\left(x \cdot p_{2}\right)+2 x_{\mu} x_{\nu} p_{2}^{2}
$$

enquanto que para os dois últimos, é igual a

$$
\begin{aligned}
\Pi_{\mu \nu}^{(2)}(q)= & \frac{i\left\langle g_{s}^{2} G^{2}\right\rangle}{2^{11} \pi^{12}} \int d^{4} x d^{4} p_{1} d^{4} p_{2} \frac{e^{i q x-i\left(p_{1}+p_{2}\right) x}}{x^{6}\left(p_{1}^{2}-m_{c}^{2}\right)\left(p_{2}^{2}-m_{c}^{2}\right)^{2}}\left[\left(x_{\nu} p_{2 \mu}+x_{\mu} p_{2 \nu}\right)\left(x \cdot p_{1}\right)+\right. \\
& \left.6 m_{c}^{2} x_{\mu} x_{\nu}+\left(3 x_{\nu} p_{1 \mu}+3 x_{\mu} p_{1 \nu}\right)\left(x \cdot p_{2}\right)-2 g_{\mu \nu}\left(x \cdot p_{1}\right)\left(x \cdot p_{2}\right)\right]
\end{aligned}
$$

Resolvendo as integrais em (3.35) e (3.36) e escrevendo a soma dos resultados em termos de uma relação de dispersão, determinamos dessa maneira, a contribuição dos Condensados de Glúons dada pela Equação (3.37) para a densidade espectral.

$$
\begin{gathered}
\rho^{\left\langle G^{2}\right\rangle}(s)=\frac{\left\langle g_{s}^{2} G^{2}\right\rangle}{2^{12} 3 \pi^{3}} \int_{\alpha_{\min }}^{\alpha_{\max }} \frac{d \alpha}{\alpha^{3}} \int_{\beta_{\min }}^{1-\alpha} \frac{d \beta}{\beta}\left\{6 \alpha(1-2 \alpha-2 \beta) F^{2}(\alpha, \beta)+\right. \\
\left.-3 m_{c}^{2}(1-\alpha-\beta)[1+\alpha(1-2 \alpha+2 \beta)-\beta(\alpha-3 \beta)] F(\alpha, \beta)-m_{c}^{4} \beta(1-\alpha-\beta)^{3}\right\} .
\end{gathered}
$$

\subsubsection{Diagramas do Condensado Misto}

\section{Diagramas Fatorizáveis}

Os diagramas associados à parte não-perturbativa fatorizável do Condensado Misto estão esquematizados na Figura 3.5.

Para os diagramas sem correção de massa, obtemos

$$
\Pi_{\mu \nu}(q)=\frac{3 m_{c}\langle\bar{s} G s\rangle}{2^{11} \pi^{10}} \int d^{4} x d^{4} p_{1} d^{4} p_{2} \frac{e^{i q x-i\left(p_{1}+p_{2}\right) x}}{x^{2}\left(p_{1}^{2}-m_{c}^{2}\right)\left(p_{2}^{2}-m_{c}^{2}\right)}\left[x_{\mu}\left(p_{1 \nu}+p_{2 \nu}\right)+x_{\nu}\left(p_{1 \mu}+p_{2 \mu}\right)\right] .
$$

Para os diagramas que representam correção de massa, temos

$$
\begin{aligned}
& \Pi_{\mu \nu}^{m_{s}}(q)=\frac{i m_{s}\langle\bar{s} G s\rangle}{2^{12} \pi^{10}} \int d^{4} x d^{4} p_{1} d^{4} p_{2} \frac{e^{i q x-i\left(p_{1}+p_{2}\right) x}}{x^{2}\left(p_{1}^{2}-m_{c}^{2}\right)\left(p_{2}^{2}-m_{c}^{2}\right)}\left[\left(x \cdot p_{1}\right)\left(x_{\mu} p_{2 \nu}+x_{\nu} p_{2 \mu}\right)+\right. \\
& \left.+2 m_{c}^{2}\left(x_{\mu} x_{\nu}-3 x^{2} g_{\mu \nu}\right)+\left(x \cdot p_{2}\right)\left(x_{\mu} p_{1 \nu}+x_{\nu} p_{1 \mu}\right)-2 g_{\mu \nu}\left(x \cdot p_{1}\right)\left(x \cdot p_{2}\right)-3 x^{2}\left(p_{1 \mu} p_{2 \nu}+p_{1 \nu} p_{2 \mu}\right)\right] .
\end{aligned}
$$



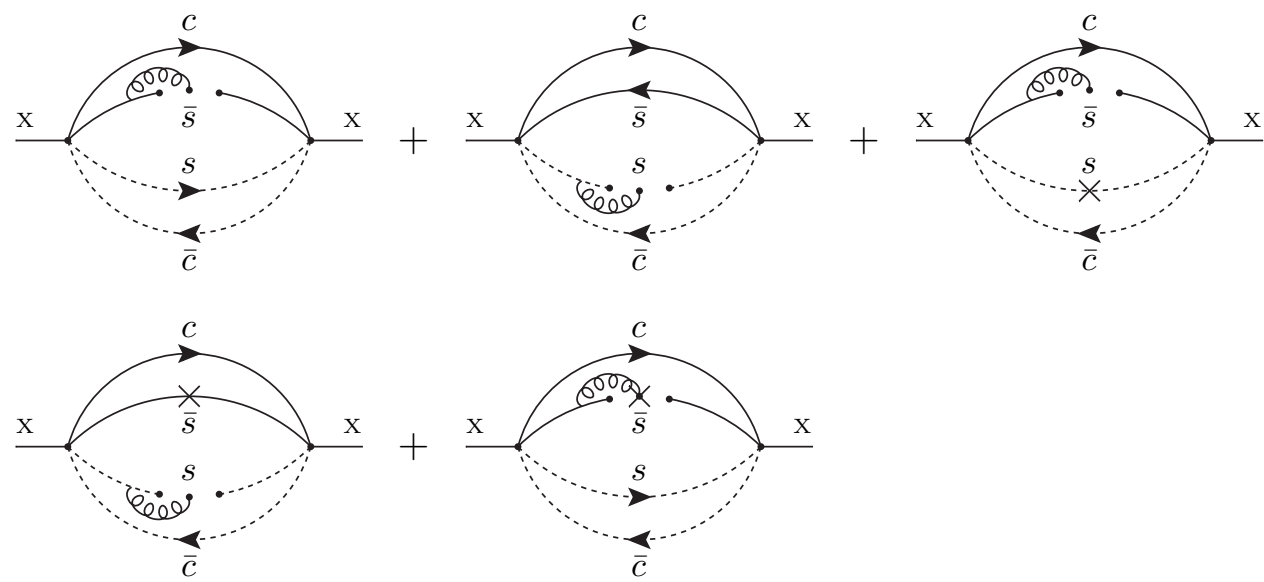

Figura 3.5: Diagramas fatorizáveis associados ao Condensados Misto.

Assim, as contribuições dos diagramas respectivamente sem correção e com correção de massa para a densidade espectral, podem ser escritas da seguinte maneira:

$$
\begin{gathered}
\rho^{m i x}(s)=\frac{3 m_{c}\langle\bar{s} G s\rangle}{2^{7} \pi^{4}} \int_{\alpha_{\min }}^{\alpha_{\max }} \frac{d \alpha}{\alpha} \int_{\beta_{\min }}^{1-\alpha} \frac{d \beta}{\beta} E(\alpha, \beta) ; \\
\rho^{m_{s} \cdot m i x}(s)=\frac{m_{s}\langle\bar{s} G s\rangle}{2^{8} \pi^{4}}\left[m_{c}^{2} \int_{\alpha_{\min }}^{\alpha_{\max }} d \alpha \int_{\beta_{\min }}^{1-\alpha} d \beta E(\alpha, \beta)-\int_{\alpha_{\min }}^{\alpha_{\max }} d \alpha\left\{m_{c}^{2}+7 \alpha(1-\alpha) s\right\}\right] .
\end{gathered}
$$




\section{Diagramas Não-Fatorizáveis}

A Figura 3.6 apresenta um esquema dos diagramas não-fatorizáveis associados ao Condensado Misto.
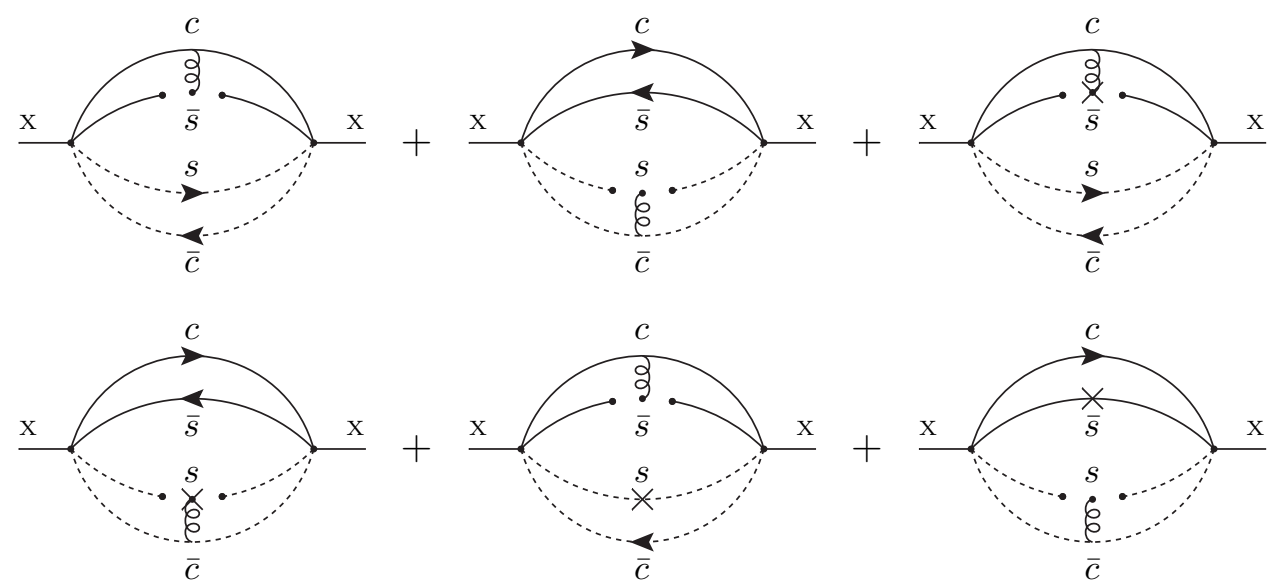

Figura 3.6: Diagramas Não-Fatorizáveis associados ao Condensado Misto.

As contribuições obtidas dos diagramas sem correção de massa, é dada pela equação abaixo

$$
\begin{aligned}
\Pi_{\mu \nu}(q)=\frac{-3 m_{c}\langle\bar{s} G s\rangle}{2^{9} \pi^{10}} \int d^{4} x d^{4} p_{1} d^{4} p_{2} \frac{e^{i q x-i\left(p_{1}+p_{2}\right) x}}{x^{4}\left(p_{1}^{2}-m_{c}^{2}\right)\left(p_{2}^{2}-m_{c}^{2}\right)}\left[x_{\mu}\left(2 p_{1 \nu}+p_{2 \nu}\right)+\right. \\
\left.+x_{\nu}\left(2 p_{1 \mu}+p_{2 \mu}\right)-2 g_{\mu \nu}\left(x \cdot p_{1}\right)\right]
\end{aligned}
$$

Para os diagramas com correção de massa:

$$
\begin{aligned}
& \Pi_{\mu \nu}^{m_{s}}(q)=\frac{-i m_{s}\langle\bar{s} G s\rangle}{2^{10} \pi^{10}} \int d^{4} x d^{4} p_{1} d^{4} p_{2} \frac{e^{i q x-i\left(p_{1}+p_{2}\right) x}}{x^{4}\left(p_{1}^{2}-m_{c}^{2}\right)\left(p_{2}^{2}-m_{c}^{2}\right)^{2}}\left[\left(x \cdot p_{1}\right)\left(x_{\mu} p_{2 \nu}+x_{\nu} p_{2 \mu}\right)+\right. \\
& \left.+6 m_{c}^{2}\left(x_{\mu} x_{\nu}-x^{2} g_{\mu \nu}\right)+3\left(x \cdot p_{2}\right)\left(x_{\mu} p_{1 \nu}+x_{\nu} p_{1 \mu}\right)-2 g_{\mu \nu}\left(x \cdot p_{1}\right)\left(x \cdot p_{2}\right)-3 x^{2}\left(p_{1 \mu} p_{2 \nu}+p_{1 \nu} p_{2 \mu}\right)\right] .
\end{aligned}
$$

Portanto, as contribuições para a densidade espectral dos diagramas não-fatorizáveis do Condensado Misto são:

$$
\rho^{m i x}(s)=\frac{3 m_{c}\langle\bar{s} G s\rangle}{2^{8} \pi^{4}} \int_{\alpha_{\min }}^{\alpha_{\max }} \frac{d \alpha}{\alpha^{2}} \int_{\beta_{\min }}^{1-\alpha} \frac{d \beta}{\beta}[\alpha(1-\alpha)-\beta(3 \alpha+2 \beta)] E(\alpha, \beta) ;
$$


$\rho^{m_{s} . m i x}(s)=\frac{m_{s}\langle\bar{s} G s\rangle}{2^{8} \pi^{4}}\left\{\int_{\alpha_{\min }}^{\alpha_{\max }} \frac{d \alpha}{\alpha} \int_{\beta_{\min }}^{1-\alpha} d \beta\left[4 m_{c}^{2}(\alpha+\beta)+3 m_{c}^{2}-\alpha \beta s\right]-2 \int_{\alpha_{\min }}^{\alpha_{\max }} d \alpha H(\alpha)\right\}$.

A contribuição total do Condensado Misto para a densidade espectral é:

$$
\rho^{m i x}(s)=\frac{3 m_{c}\langle\bar{s} G s\rangle}{2^{8} \pi^{4}} \int_{\alpha_{\min }}^{\alpha_{\max }} \frac{d \alpha}{\alpha^{2}} \int_{\beta_{\min }}^{1-\alpha} \frac{d \beta}{\beta}[\alpha(1-\alpha)-\beta(5 \alpha+2 \beta)] F(\alpha, \beta),
$$

para os diagramas sem correção de massa e

$$
\begin{aligned}
\rho^{m_{s} \cdot \operatorname{mix}}(s) & =\frac{m_{s}\langle\bar{s} G s\rangle}{2^{8} \pi^{4}}\left\{\int_{\alpha_{\min }}^{\alpha_{\max }} \frac{d \alpha}{\alpha} \int_{\beta_{\min }}^{1-\alpha} d \beta\left[m_{c}^{2}(3+5 \alpha+4 \beta)-\alpha \beta s\right]+\right. \\
& \left.-\int_{\alpha_{\min }}^{\alpha_{\max }} \frac{d \alpha}{\alpha}\left[m_{c}^{2}(2+\alpha)-\alpha(1-\alpha)(2-7 \alpha) s\right]\right\},
\end{aligned}
$$

para os diagramas com correção de massa.

\subsubsection{Diagramas dos Condensados de 4-Quarks}

A Figura 3.7 apresenta os diagramas associados ao Condensado de 4-Quarks.

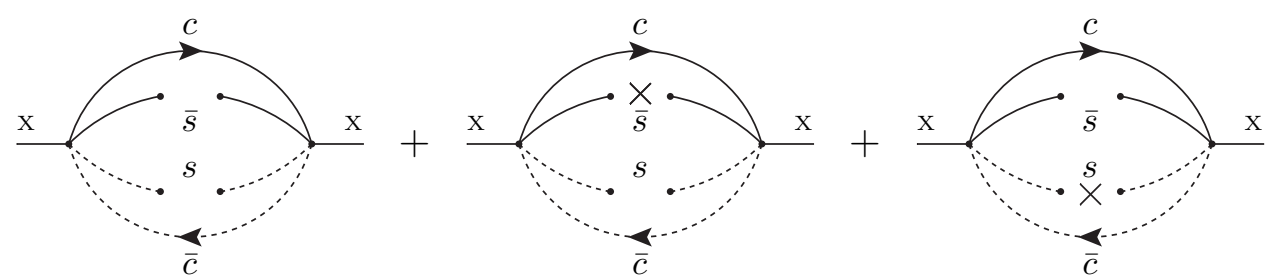

Figura 3.7: Diagramas associados aos Condensados de 4-Quarks.

Substituindo os propagadores (Apêndice X) na Equação (3.15), obtemos para o diagrama sem correção de massa a seguinte expressão

$$
\Pi_{\mu \nu}(q)=-\frac{i\langle\bar{s} s\rangle^{2}}{2^{9} \pi^{8}} \int d^{4} x d^{4} p_{1} d^{4} p_{2} \frac{e^{i q x-i\left(p_{1}+p_{2}\right) x}}{\left(p_{1}^{2}-m_{c}^{2}\right)\left(p_{2}^{2}-m_{c}^{2}\right)^{2}}\left[2 g_{\mu \nu} m_{c}^{2}+p_{1 \mu} p_{2 \nu}+p_{1 \nu} p_{2 \mu}\right],
$$


enquanto que para os diagramas com correção de massa resulta na Equação (3.49), $\Pi_{\mu \nu}^{m_{s}}(q)=\frac{m_{c} m_{s}\langle\bar{s} s\rangle^{2}}{2^{10} \pi^{8}} \int d^{4} x d^{4} p_{1} d^{4} p_{2} \frac{e^{i q x-i\left(p_{1}+p_{2}\right) x}}{\left(p_{1}^{2}-m_{c}^{2}\right)\left(p_{2}^{2}-m_{c}^{2}\right)^{2}}\left[x_{\mu}\left(p_{1 \nu}+p_{2 \nu}\right)+x_{\nu}\left(p_{1 \mu}+p_{2 \mu}\right)\right]$.

Deste modo, resolvendo as integrais acima, podemos escrever os resultados de tal maneira a obtermos as contribuições dos diagramas para a densidade espectral. Fazendo isso, encontramos

$$
\begin{gathered}
\rho^{\langle\bar{s} s\rangle^{2}}(s)=-\frac{\langle\bar{s} s\rangle^{2}}{2^{6} 3 \pi^{2}}\left(8 m_{c}^{2}+s\right) \sqrt{1-4 m_{c}^{2} / s} \\
\rho^{m_{s} \cdot\langle\bar{s} s\rangle^{2}}(s)=-\frac{m_{c} m_{s}\langle\bar{s} s\rangle^{2}}{2^{5} \pi^{2}} \sqrt{1-4 m_{c}^{2} / s}
\end{gathered}
$$

\subsubsection{Condensados de Dimensão 8}

Por questão de simplicidade, vamos separar as contribuições dos diagramas da Figura 3.8 em quatro, são elas: $\Pi_{A \mu \nu}^{8}(q), \Pi_{B \mu \nu}^{8}(q), \Pi_{A \mu \nu}^{m_{s} .8}(q)$ e $\Pi_{B \mu \nu}^{m_{s} .8}(q)$ onde,

$$
\begin{aligned}
\Pi_{A \mu \nu}^{8}(q) & =-i \frac{\langle\bar{s} s\rangle\langle\bar{s} G s\rangle}{2^{12} \pi^{8}} \int d^{4} x d^{4} p_{1} d^{4} p_{2} \frac{e^{i q x-i\left(p_{1}+p_{2}\right) x}}{\left(p_{1}^{2}-m_{c}^{2}\right)\left(p_{2}^{2}-m_{c}^{2}\right)} x^{2}\left[2 m_{c}^{2} g_{\mu \nu}+p_{1 \mu} p_{2 \nu}+\right. \\
& \left.+p_{1 \nu} p_{2 \mu}\right] ; \\
\Pi_{B \mu \nu}^{8}(q) & =i \frac{\langle\bar{s} s\rangle\langle\bar{s} G s\rangle}{2^{10} \pi^{8}} \int d^{4} x d^{4} p_{1} d^{4} p_{2} \frac{e^{i q x-i\left(p_{1}+p_{2}\right) x}}{\left(p_{1}^{2}-m_{c}^{2}\right)\left(p_{2}^{2}-m_{c}^{2}\right)^{2}}\left[2 m_{c}^{2} g_{\mu \nu}+p_{1 \mu} p_{2 \nu}+p_{1 \nu} p_{2 \mu}\right] \\
\Pi_{A \mu \nu}^{m_{s} .8}(q) & =\frac{5 m_{c} m_{s}\langle\bar{s} s\rangle\langle\bar{s} G s\rangle}{2^{14} 3 \pi^{8}} \int d^{4} x d^{4} p_{1} d^{4} p_{2} \frac{e^{i q x-i\left(p_{1}+p_{2}\right) x}}{\left(p_{1}^{2}-m_{c}^{2}\right)\left(p_{2}^{2}-m_{c}^{2}\right)} x^{2}\left[x_{\mu}\left(p_{1 \nu}+p_{2 \nu}\right)+\right. \\
& \left.+x_{\nu}\left(p_{1 \mu}+p_{2 \mu}\right)\right] ; \\
\Pi_{B \mu \nu}^{m_{s} .8}(q) & =\frac{-m_{c} m_{s}\langle\bar{s} s\rangle\langle\bar{s} G s\rangle}{2^{12} 3 \pi^{8}} \int d^{4} x d^{4} p_{1} d^{4} p_{2} \frac{e^{i q x-i\left(p_{1}+p_{2}\right) x}}{\left(p_{1}^{2}-m_{c}^{2}\right)\left(p_{2}^{2}-m_{c}^{2}\right)}\left[-6 g_{\mu \nu}\left(x . p_{1}\right)\right. \\
& \left.+8 g_{\mu \nu}\left(x \cdot p_{2}\right)+2 x_{\mu}\left(12 p_{1 \nu}+5 p_{2 \nu}\right)\right] .
\end{aligned}
$$



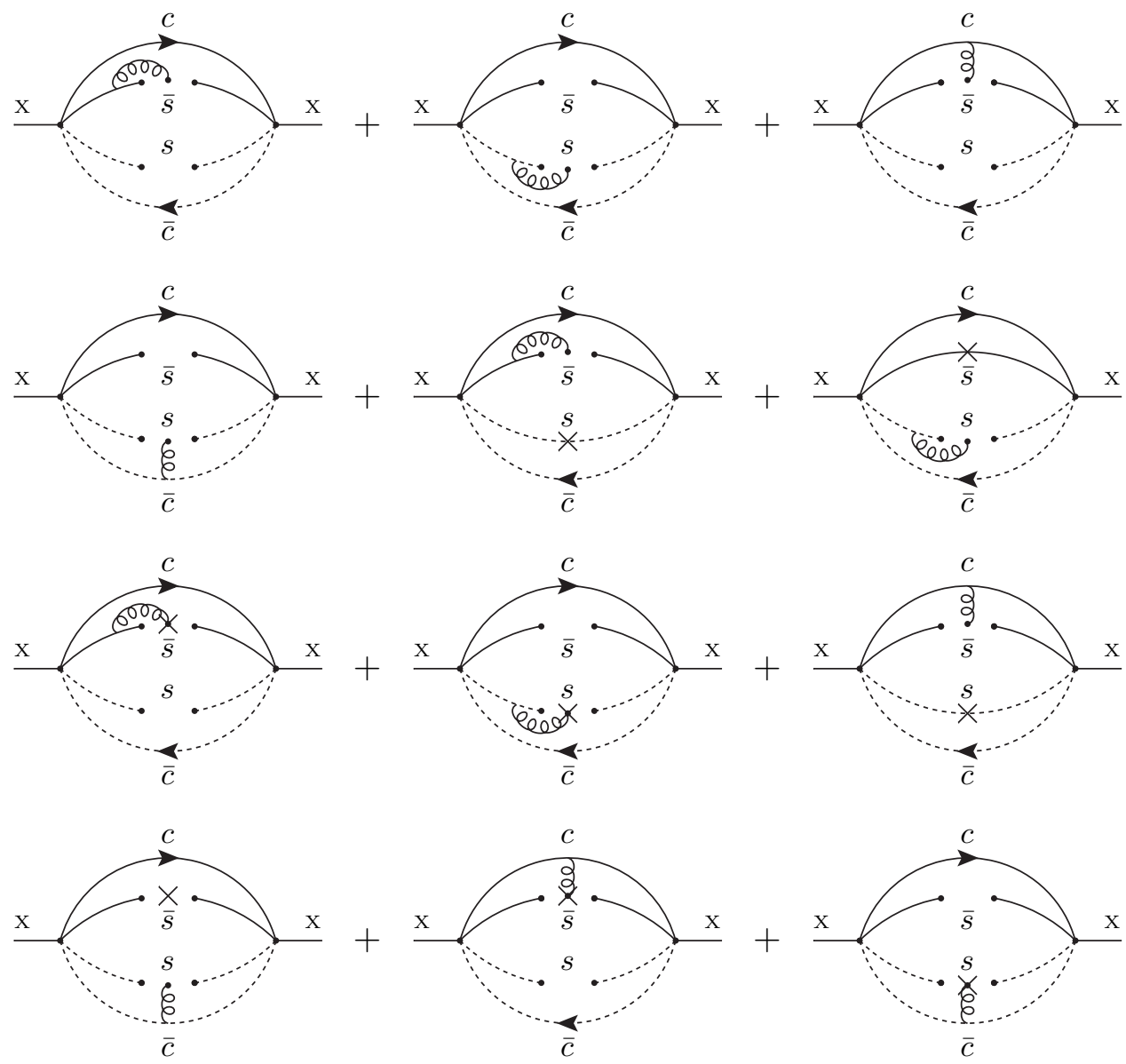

Figura 3.8: Diagramas associados à dimensão 8 da OPE.

Somando os resultados das integrais $\Pi_{A \mu \nu}^{8}(q)$ e $\Pi_{B \mu \nu}^{8}(q)$, obtemos um termo que pode ser escrito em termos de uma relação de dispersão com

$$
\rho^{8}(s)=\frac{m_{c}^{2}\langle\bar{s} s\rangle\langle\bar{s} g \sigma \cdot G s\rangle}{2^{6} \pi^{2}} \frac{\sqrt{1-4 m_{c}^{2} / s}}{s},
$$

e um outro que após uma Transformada de Borel (Capítulo 1), resulta em

$$
\Pi^{8}\left(M^{2}\right)=-\frac{m_{c}^{2}\langle\bar{s} s\rangle\langle\bar{s} g \sigma \cdot G s\rangle}{2^{6} \pi^{2}} \int_{0}^{1} \frac{d \alpha}{(1-\alpha)} e^{-\frac{m_{c}^{2}}{\alpha(1-\alpha) M^{2}}}\left[1-3 \alpha+\frac{2 m_{c}^{2}}{\alpha M^{2}}\right] .
$$

Para as integrais $\Pi_{A \mu \nu}^{m_{s} .8}(q)$ e $\Pi_{B \mu \nu}^{m_{s} .8}(q)$, obtemos uma expressão sem parte imaginária, assim, aplicando uma Transformada de Borel ao resultado da soma das Eq.s (3.54) 
e (3.55) encontramos

$$
\begin{aligned}
\Pi^{m_{s} .8}\left(M^{2}\right) & =\frac{m_{c} m_{s}\langle\bar{s} s\rangle\langle\bar{s} g \sigma \cdot G s\rangle}{2^{6} \pi^{2}} \int_{0}^{1} \frac{d \alpha}{\alpha} e^{-\frac{m_{c}^{2}}{\alpha(1-\alpha) m^{2}}}\left[\frac{m_{c}^{2}}{M^{2}} \frac{\left(6-4 \alpha-10 \alpha^{2}\right)}{\alpha(1-\alpha)}+\right. \\
& \left.-\left(6-13 \alpha+20 \alpha^{2}\right)\right] .
\end{aligned}
$$

Finalmente, podemos determinar a expressão que será comparada com a função de correlação calculada pelo Lado Fenomenológico. A densidade espectral total será a soma das densidades espectrais associadas a cada dimensão da OPE. Entretanto, nos diagramas de dimensão 8 as expressões (2.58) e (2.59) não estão escritas em termos de uma relação de dispersão, mas, estas equações são integradas diretamente da relação de dispersão. Assim, a Equação (2.82) será modificada da seguinte maneira:

$$
-\lambda^{2} e^{-m^{2} / M^{2}}=\int_{s_{\min }}^{s_{0}} d s \rho^{O P E}(s) e^{-s / M^{2}}+\Pi^{8}\left(M^{2}\right)+\Pi^{m_{s} .8}\left(M^{2}\right) .
$$

\subsection{Lado Fenomenológico}

Obtidas as contribuições do Lado da OPE, podemos agora construir o Lado Fenomenológico das Regras de Soma para o X(4350). Para isso, nós parametrizamos o acoplamento do méson vetorial $X$ com a corrente descrita pela Equação (3.3) da seguinte maneira:

$$
\left\langle 0\left|j_{\mu}\right| X\right\rangle=\lambda \epsilon_{\mu}
$$

Deste modo, podemos reescrever a função de correlação para o Lado Fenomenológico (2.77) como

$$
\Pi^{f e n}\left(q^{2}\right)=\lambda^{2} e^{-m^{2} / M^{2}}+\int_{s_{0}}^{\infty} d s \rho^{O P E}(s) e^{-s / M^{2}} .
$$

Determinado o Lado Fenomenológico, podemos compará-lo com o Lado da OPE devido ao Princípio da Dualidade Quark-Hádron e assim, determinarmos a massa do estado representado pela corrente na Equação (3.3). 


\subsection{Regra de Soma}

Neste momento, podemos fazer uso do Princípio da Dualidade Quark-Hádron que nos permite igualar a função de correlação calculada pelo Lado Fenomenológico e pelo Lado da OPE. Deste modo, igualando ambas as descrições da função de correlação, obtemos

$$
\lambda^{2} e^{-m^{2} / M^{2}}=\int_{s_{\min }}^{s_{0}} d s \rho^{O P E}(s) e^{-s / M^{2}}+\Pi^{8}\left(M^{2}\right)+\Pi^{m_{s} .8}\left(M^{2}\right) .
$$

Derivando a equação acima com respeito a $\frac{1}{M^{2}}$, obtemos

$$
\lambda^{2} m^{2} e^{-m^{2} / M^{2}}=\int_{s_{\min }}^{s_{0}} d s \rho^{O P E} s(s) e^{-s / M^{2}}-\frac{d \Pi^{8}\left(M^{2}\right)}{d\left(1 / M^{2}\right)}-\frac{d \Pi^{m_{s} \cdot 8}\left(M^{2}\right)}{d\left(1 / M^{2}\right)},
$$

onde

$$
\begin{gathered}
\frac{d \Pi^{8}\left(M^{2}\right)}{d\left(1 / M^{2}\right)}=\frac{m_{c}^{4}\langle\bar{s} s\rangle\langle\bar{s} g \sigma G s\rangle}{2^{6} \pi^{2}}\left\{\int_{0}^{1} \frac{d \alpha}{\alpha(1-\alpha)} e^{\frac{-m_{c}^{2}}{M^{2} \alpha(1-\alpha)}}\left[2-\frac{1}{(1-\alpha)}\left(1+\frac{2 m_{c}^{2}}{\alpha M^{2}}-3 \alpha\right)\right]\right\} ; \\
-\frac{d \Pi^{m_{s} .8}\left(M^{2}\right)}{d\left(1 / M^{2}\right)}=\frac{m_{c}^{3} m_{s}\langle\bar{s} s\rangle\langle\bar{s} g \sigma G s\rangle}{3 \pi^{2} 2^{7}}\left\{\int _ { 0 } ^ { 1 } \frac { d \alpha } { \alpha ^ { 2 } ( 1 - \alpha ) } e ^ { \frac { - m _ { c } ^ { 2 } } { M ^ { 2 } \alpha ( 1 - \alpha ) } } \left[\left(12-17 \alpha+10 \alpha^{2}\right)+\right.\right. \\
\left.\left.-\frac{m_{c}^{2}}{M^{2}} \frac{6-4 \alpha-10 \alpha^{2}}{\alpha(1-\alpha)}\right]\right\} .
\end{gathered}
$$

Portanto, a Equação (2.84) será reescrita da seguinte maneira

$$
m^{2}=\frac{\int_{4 m_{c}^{2}}^{s_{0}} d s s \rho^{O P E}(s) e^{-s / M^{2}}-\frac{d \Pi^{8}\left(M^{2}\right)}{d\left(1 / M^{2}\right)}-\frac{d \Pi^{m_{s} \cdot 8}\left(M^{2}\right)}{d\left(1 / M^{2}\right)}}{\int_{s_{4 m_{c}^{2}}}^{s_{0}} d s \rho^{O P E}(s) e^{-s / M^{2}}+\Pi^{8}\left(M^{2}\right)+\Pi^{m_{s} \cdot 8}\left(M^{2}\right)} .
$$

É através da Equação (3.64) que calcularemos a massa do estado. As integrais presentes nesta equação não possuem solução analítica, sendo assim, elas serão resolvidas numericamente.

Na próxima seção detalharemos os valores numéricos dos condensados e massas dos quarks. Uma análise sobre a convergência da OPE e a dominância do pólo sobre o contínuo será feita, para que possamos determinar uma Janela de Borel e consequentemente resultados confiáveis para a massa desse estado. 


\subsection{Resultados Numéricos}

Para uma consistente comparação de nossos resultados com outros trabalhos sobre estados moleculares usando regras de soma, vamos usar os mesmos valores para as massas dos quarks e condensados utilizados nas referências [44, 49, 50, 51, $13,52,53,54]$ :

$$
\begin{gathered}
m_{c}\left(m_{c}\right)=(1.23 \pm 0.05) \mathrm{GeV} ; \\
m_{s}=(0.13 \pm 0.03) \mathrm{GeV} ; \\
\langle\bar{q} q\rangle=-(0.23 \pm 0.03)^{3} \mathrm{GeV}^{3} ; \\
\langle\bar{s} s\rangle=0.8\langle\bar{q} q\rangle ; \\
\langle\bar{s} g \sigma G s\rangle=m_{0}^{2}\langle\bar{s} s\rangle ; \\
\left\langle g^{2} G^{2}\right\rangle=0.88 \mathrm{GeV}^{4} ; \\
m_{0}^{2}=0.8 \mathrm{Gev}^{2} .
\end{gathered}
$$

No Capítulo 2 mencionamos a importância da Janela de Borel, região onde os resultados apresentam uma certa confiabilidade. Para determinarmos essa região, fixamos um valor máximo e um mínimo para a Massa de Borel $M^{2}$. Tais valores são fixados analisando a convergência da OPE e a dominância do pólo sobre o contínuo.

O valor mínimo da Massa de Borel é fixado estabelecendo que a contribuição dos condensados de dimensão 8 deve ser menor que $20 \%$ da contribuição total, isto é, a contribuição de todas as outras dimensões da OPE. A Figura 3.9 apresenta todas as contribuições relativas da OPE. Note que para $M^{2} \geq 2.8 \mathrm{GeV}^{2}$ a contribuição dos condensados de dimensão 8 é menor do que $10 \%$ da contribuição total.

Por outro lado, sabemos que a Massa de Borel é um parâmetro livre dentro das Regras de Soma, que surge devido à Transformada de Borel. Em uma situação ideal não existiria dependência em relação à Massa de Borel, porém, como foi dito no Capítulo 2 uma dependência é introduzida nos cálculos devido ao truncamento da OPE e suposições fenomenológicas. Portanto, da Figura 3.10 observamos que uma 

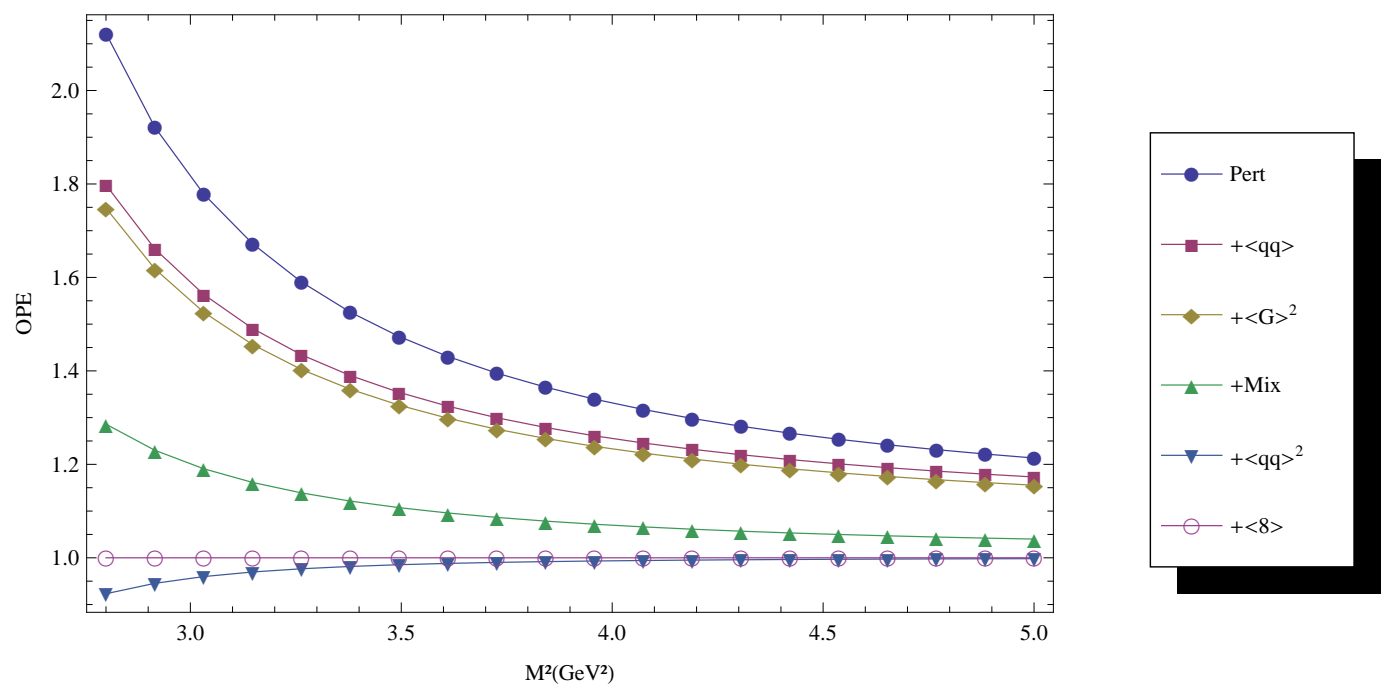

Figura 3.9: Comportamento da série devido ao acréscimo das contribuições relativas de cada um dos condensados. O cálculo foi realizado para $\sqrt{s_{0}}=5.45 \mathrm{GeV}$.

boa estabilidade na Massa de Borel é obtida somente para $M^{2} \geq 3.2 \mathrm{GeV}^{2}$. Assim, vamos fixar esse valor para o mínimo da Massa de Borel, isto é, $M_{\min }^{2}=3.2 \mathrm{GeV}^{2}$.

O valor máximo pode ser determinado pela Figura 3.11. Como estamos calculando a massa do estado fundamental, devemos trabalhar numa região de $M^{2}$ de tal maneira que a contribuição do pólo seja maior ou no mínimo igual, à contribuição do contínuo. Devido à dominância da contribuição perturbativa, a contribuição do contínuo aumenta com $M^{2}$, assim, estabelecemos que o valor máximo para a Massa de Borel será aquele em que as contribuições do pólo e do contínuo se igualam, portanto, da Figura 3.11 vemos que esse valor é $M_{\max }^{2}=3.74 \mathrm{GeV}^{2}$.

O valor de $s_{0}$ (limiar do contínuo) inicialmente é escolhido de forma arbitrária. Se a diferença entre este valor e a massa do méson calculada for igual a $0.5 \mathrm{GeV}$, então, o valor inicial de $s_{0}$ é aceito. A Tabela 3.2 apresenta os valores de $M_{\text {max }}^{2}$ para outros valores de $\sqrt{s_{0}}$.

Determinada a Janela de Borel para cada valor de $s_{0}$, então, podemos calcular a massa do estado em questão. Variando o limiar do contínuo no intervalo $5.3 \leq$ 


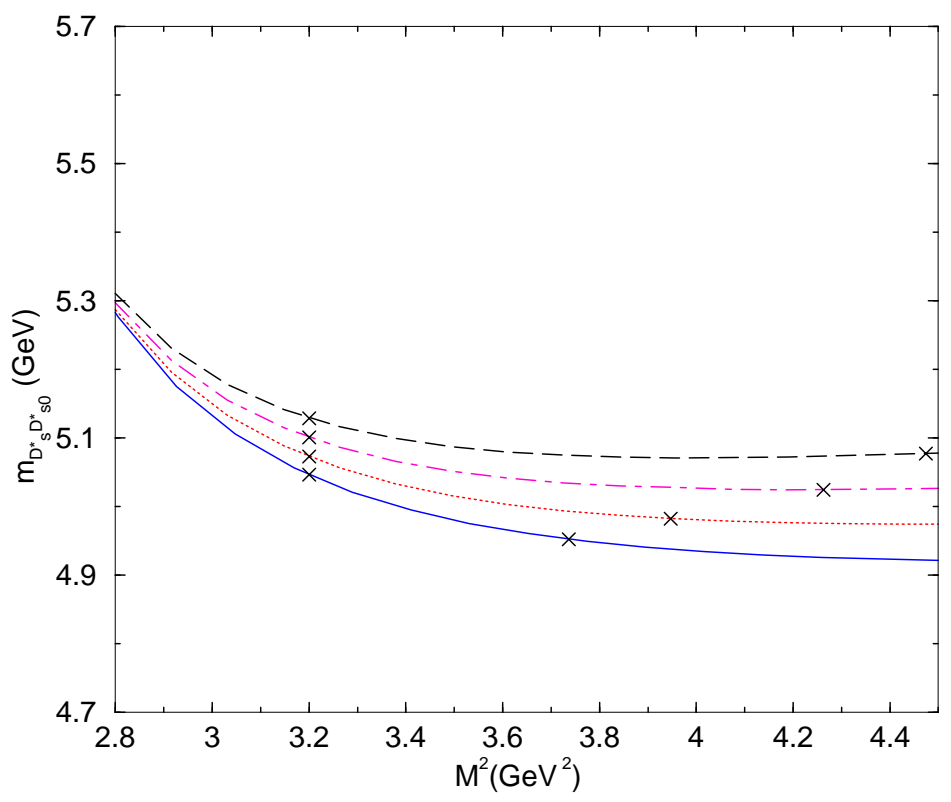

Figura 3.10: Massa calculada para o estado $D_{s}^{*} D_{s 0}^{*}$ em função da Massa de Borel ao quadrado $M^{2}$, para diferentes valores de $\sqrt{s_{0}}$. O símbolo $\times$ em cada curva representa os valores máximo e mínimo da Janela de Borel.

$\sqrt{s_{0}} \leq 5.6 \mathrm{GeV}$, obtemos

$$
m_{D_{s}^{*} D_{s 0}^{*}}=(5.09 \pm 0.09) \mathrm{GeV}
$$

Podemos aumentar o intervalo dos valores da massa do quark charmoso de acordo com o PDG: $1.16 \leq m_{c} \leq 1.34 \mathrm{GeV}$. Novamente, calculando a massa do méson exótico $D_{s}^{*} D_{s 0}^{*}$ considerando essa variação na massa do quark $c$, temos

$$
m_{D_{s}^{*} D_{s 0}^{*}}=(5.11 \pm 0.18) \mathrm{GeV}
$$

Como a contribuição do contínuo aumenta com $m_{c}$, não é possível determinarmos uma Janela de Borel para valores de $m_{c}$ acima de $1.42 \mathrm{GeV}$.

Observe que o valor dado por (3.73) está muito distante do valor experimental para a massa do $X(4350)$. Isto implica que a molécula $D_{s}^{*} D_{s 0}^{*}$ descrita pela corrente (3.3) consistente com os números quânticos $1^{-+}$, não representa a estrutura estreita $X(4350)$. 

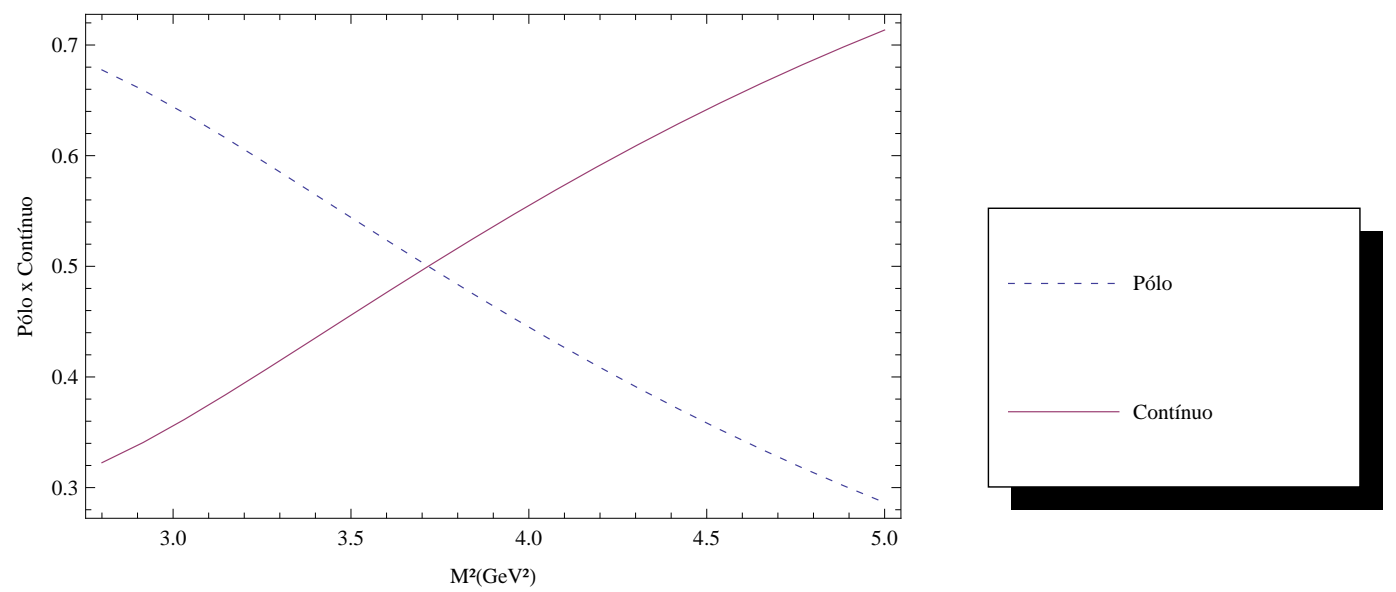

Figura 3.11: Contribuição do Pólo versus Contínuo para $\sqrt{s_{0}}=5.3 \mathrm{GeV}$.

Tabela 3.2: Limite superior da Janela de Borel $M_{\max }^{2}$ associado com alguns valores de $\sqrt{s_{0}}$ para a Regra de Soma do $X(4350)$ supondo-o um estado exótico $1^{-+}$.

\begin{tabular}{|c|c|}
\hline$\sqrt{s_{0}}(\mathrm{GeV})$ & $M_{\max }^{2}\left(\mathrm{GeV}^{2}\right)$ \\
\hline 5.2 & 3.42 \\
\hline 5.3 & 3.74 \\
\hline 5.4 & 3.95 \\
\hline 5.5 & 4.26 \\
\hline 5.6 & 4.47 \\
\hline
\end{tabular}

Um estudo considerando uma corrente molecular $D^{*} D_{0}^{*}$ com os números quânti$\cos 1^{-+}$pode ser realizado, substituindo o quark $s$ pelos quarks leves $q=u, d$. Para isso, basta tomarmos o valor de $m_{s}$ igual a zero e substituirmos o $\langle\bar{s} s\rangle$ por $\langle\bar{q} q\rangle$ nas equações para as densidades espectrais obtidas anteriormente. Analisando a convergência da OPE através da Figura 3.12, observamos que o gráfico é bastante similar ao caso anterior, assim, temos uma boa convergência para valores de $M^{2}$ maiores do que $3.2 \mathrm{GeV}^{2}$, que também para este caso, será o valor fixado para $M_{\min }^{2}$.

Da Figura 3.13 determinamos o valor máximo da Janela de Borel. Como as 

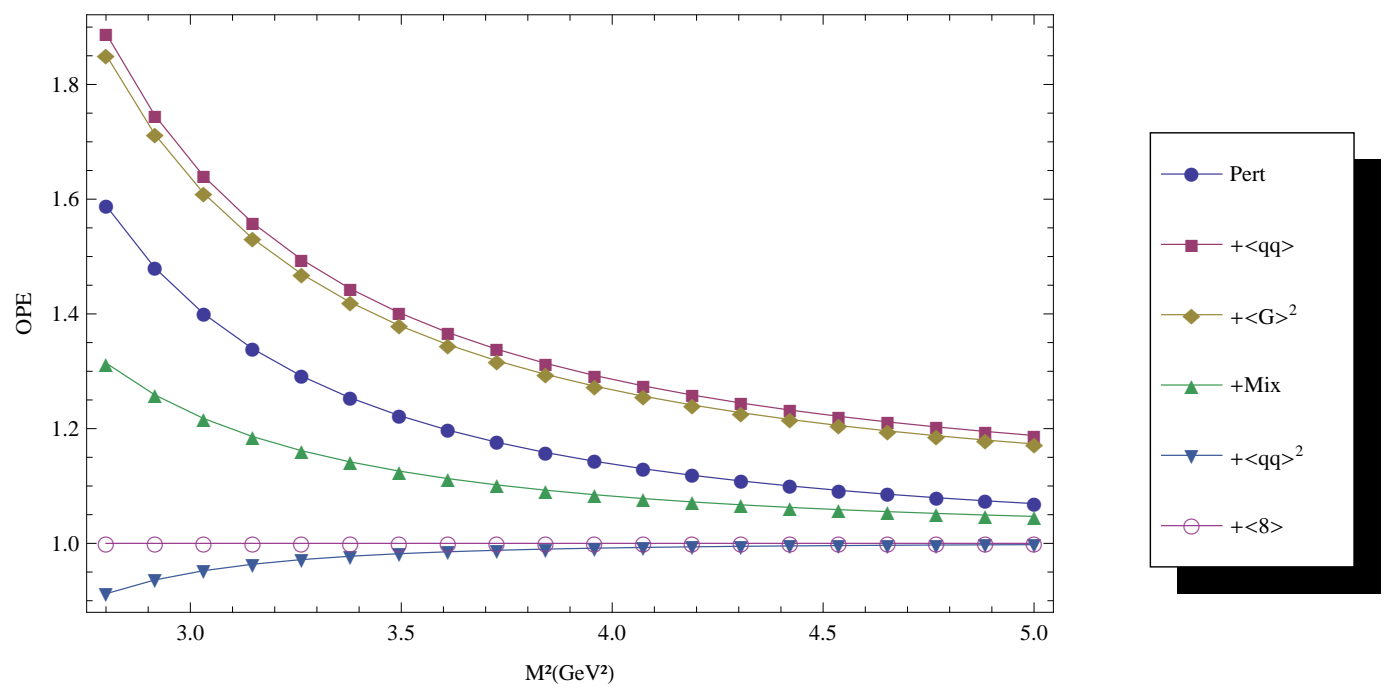

Figura 3.12: Convergência da OPE para o caso no qual estamos supondo o estado descrito pela molécula $D^{*} D_{0}^{*}$, em função de $M^{2}$.

contribuições do pólo e do contínuo se igualam para $M^{2}$ igual a $4.2 \mathrm{GeV}^{2}$, escolhemos $M_{\text {max }}^{2}=4.2 \mathrm{GeV}^{2}$.

Assim, a Janela de Borel para esse caso será $3.2 \leq M^{2} \leq 4.2 \mathrm{GeV}^{2}$. Na Figura 3.14, mostramos o resultado para a massa usando diferentes valores do limiar do contínuo. Usando os valores para o limiar do contínuo no intervalo $5.2 \leq \sqrt{s_{0}} \leq$ $5.5 \mathrm{GeV}$ obtemos para o estado descrito por uma corrente molecular $1^{-+}, D^{*} D_{0}^{*}$ o seguinte valor:

$$
m_{D^{*} D_{0}^{*}}=(4.92 \pm 0.08) \mathrm{GeV} .
$$

Observe que o valor dado por (3.74) está aproximadamente $100 \mathrm{MeV}$ abaixo do valor obtido para o caso com quark estranho. No caso da corrente molecular $D^{*} D_{0}^{*}$ com $1^{--}$, os autores da Referência [44] obtiveram uma massa igual a $m_{1^{--}}=$ $(4.27 \pm 0.10) \mathrm{GeV}$ que é muito menor do que para o caso exótico.

Além disso, é interessante observar que a massa obtida para um estado descrito por uma corrente molecular $D_{s}^{*} D_{s 0}^{*}$ com $1^{--}$é igual a $m_{1^{--}}=4.42 \pm 0.10 \mathrm{GeV}$, muito menor do que o valor obtido supondo a mesma molécula com $1^{-+}$. Isso pode ser uma indicação de que pode ser mais fácil formar estados moleculares com números 

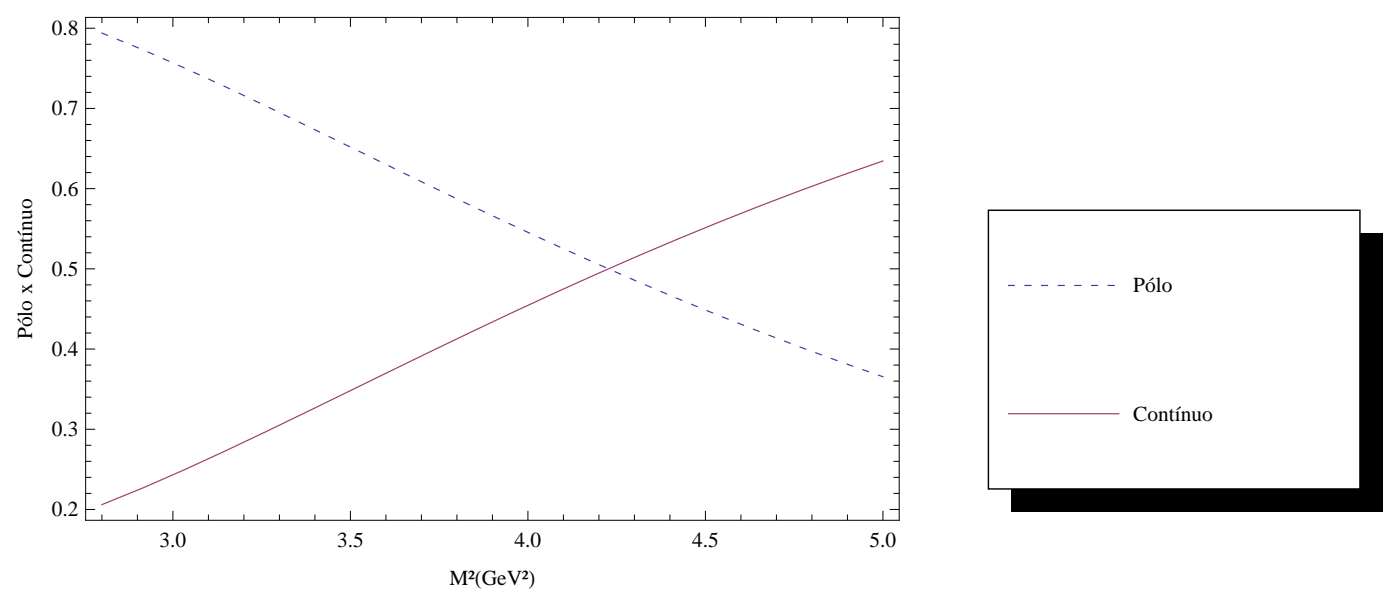

Figura 3.13: Relação Pólo versus Contínuo para $\sqrt{s_{0}}=5.4 \mathrm{GeV}$.

quânticos não-exóticos. 


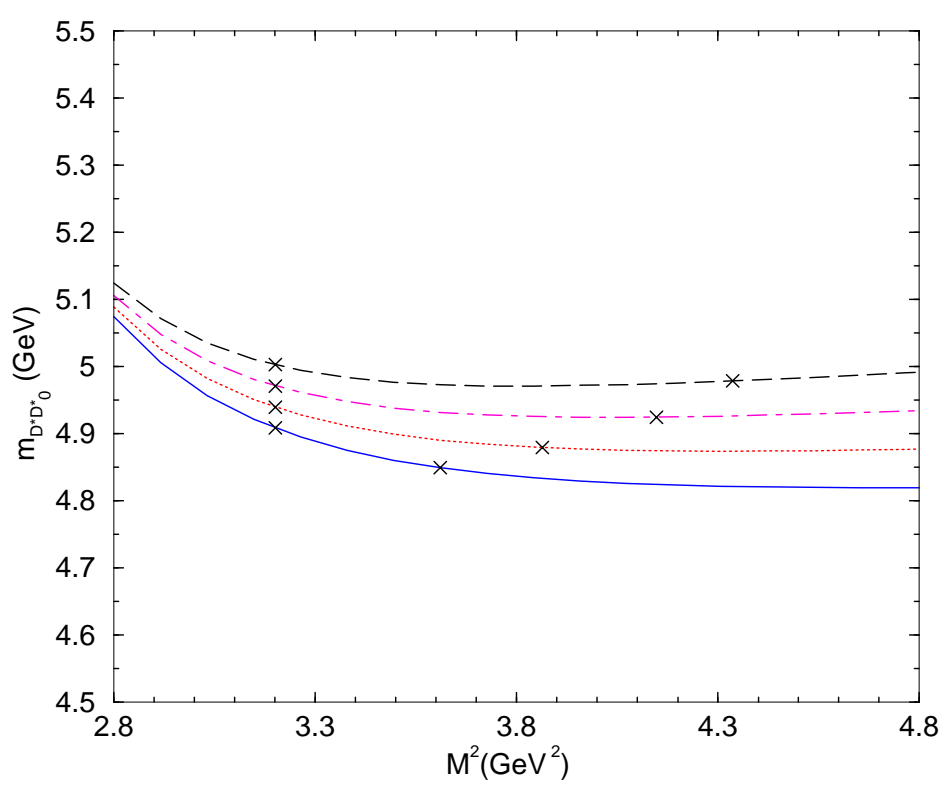

Figura 3.14: Massa associada ao méson $X(4350)$ calculada supondo uma corrente molecular $D^{*} D_{0}^{*}$, como função do parâmetro que determina o limiar do contínuo $\sqrt{s_{0}}$, para $\sqrt{s_{0}}=5.2 \mathrm{GeV}$ (linha contínua), $\sqrt{s_{0}}=5.3 \mathrm{GeV}$ (linha pontilhada), $\sqrt{s_{0}}=5.4 \mathrm{GeV}$ (linha ponto-tracejada) e $\sqrt{s_{0}}=5.5 \mathrm{GeV}$ (linha tracejada). 


\section{Capítulo 4}

\section{Conclusões}

As Regras de Soma da QCD tem se apresentado como uma ferramenta muito útil no entendimento da estrutura dos novos estados do charmonium, observados recentemente pelas colaborações Belle e BaBar. Apesar de suas limitações com respeito ao grau de precisão, as Regras de Soma fornecem evidências a favor ou contra a existência desses estados [26]. Entretanto, resultados conclusivos com maior grau de precisão podem ser alcançados quando temos acesso a mais informações experimentais sobre o estado estudado.

Neste trabalho usamos as Regras de Soma da QCD para tentar estudar o estado $X(4350)$ observado pela colaboração Belle [19]. Em particular, calculamos a massa de um estado molecular $D_{s}^{*} D_{s 0}^{*}$ descrito pela corrente dada pela Equação (3.3) consistente com os números $1^{-+}$. O valor encontrado não é compatível com o resultado experimental, indicando que o estado $X(4350)$ não pode ser representado por um estado molecular do tipo aqui considerado.

Além disso, realizamos os mesmos cálculos considerando também uma corrente molecular do tipo $D^{*} D_{0}^{*}$ com os mesmos números quânticos $1^{-+}$. Novamente os resultados não foram consistentes com o valor da massa experimental do $X(4350)$. A molécula com quark leve apresentou um valor aproximadamente $100 \mathrm{MeV}$ abaixo do caso da molécula com quark estranho.

É interessante observar que a massa obtida para um estado descrito por uma 
corrente molecular $D_{s}^{*} D_{s 0}^{*}$ com $1^{--}$é igual a $m_{1^{--}}=4.42 \pm 0.10 \mathrm{GeV}$, muito menor do que o valor obtido supondo a mesma molécula com $1^{-+}$. Isto pode ser um indício de que estados moleculares são mais facilmente formados com números quânticos não-exóticos. 


\section{Apêndice A}

\section{Propagadores}

No capítulo 1 calculamos cada termo do propagador utilizado nas Regras de Soma da QCD. Nesse capítulo os propagadores utilizados no cálculo da OPE, foram obtidos após realizarmos a expansão de Wick na função de dois pontos definida pela Equação (2.1). Ainda no mesmo capítulo, foi dito que o último termo dessa equação numa teoria perturbativo seria nulo. Por outro lado, como esse termo é avaliado no vácuo da QCD, implica que é ele que introduz as informações não-perturbativas do vácuo da QCD. Deste modo, cada termo do propagador obtido, é substituído de acordo com a construção dos diagramas que contribuem para a OPE.

A seguir listamos todos os propagadores perturbativos, não-perturbativos, fatoráveis e não fatoráveis e sua representação na forma de diagramas.

\section{A.1 Propagadores associados com quarks leves}

Os diagramas abaixo estão associados com os propagadores de quark leve $(q=$ $u, d$ e $s$ ). Os três últimos diagramas (não-fatoráveis) devem ser tratados com cuidado, pois devem sempre aparecer associados a um termo pertubativo de glúons em outro propagador, no qual devemos omitir $g_{s} G_{a b}^{n}(0)$. 


$$
\begin{aligned}
& \longrightarrow \quad \frac{i \not p \delta_{a b}}{2 \pi^{2} x^{4}} \\
& -\frac{m_{q} \delta_{a b}}{4 \pi^{2} x^{2}} \\
& -\frac{\delta_{a b}}{12}\langle\bar{q} q\rangle \\
& \frac{i \not h \delta_{a b}}{48} m_{q}\langle\bar{q} q\rangle \\
& -\frac{x^{2} \delta_{a b}}{3 \cdot 2^{6}}\left\langle\bar{q} g_{s} \sigma G q\right\rangle \\
& \frac{i x^{2} \not k \delta_{a b}}{3^{2} \cdot 2^{7}} m_{q}\left\langle\bar{q} g_{s} \sigma G q\right\rangle \\
& -\frac{i\left(\not \sigma_{\mu \nu}+\sigma_{\mu \nu} \not\right) t_{a b}^{n}}{3 \cdot 2^{9} \pi^{2} x^{2}}\left\langle g_{s}^{2} G^{2}\right\rangle \\
& -\frac{\sigma_{\mu \nu} t_{a b}^{n}}{3 \cdot 2^{6}}\left\langle\bar{q} g_{s} \sigma G q\right\rangle \\
& \frac{i\left(\not \sigma_{\mu \nu}+\sigma_{\mu \nu} \not\right) t_{a b}^{n}}{3 \cdot 2^{8}} m_{q}\left\langle\bar{q} g_{s} \sigma G q\right\rangle
\end{aligned}
$$

\section{A.2 Propagadores associados com quarks pesados}

Os diagramas a seguir estão associados com os propagadores de quarks pesados. 


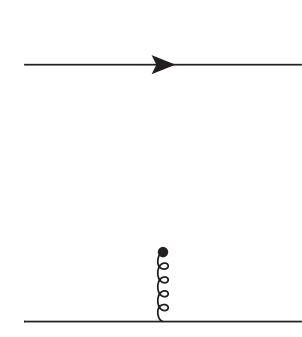

$$
\frac{i\left(\not p+m_{c}\right) \delta_{a b}}{p^{2}-m_{c}^{2}}
$$

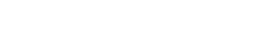

$$
-\frac{i t_{a b}^{n} g_{s} G_{\mu \nu}^{n}(0)}{4}\left[\frac{\not p \sigma^{\mu \nu}+\sigma^{\mu \nu} \not \nu+2 m_{c} \sigma^{\mu \nu}}{\left(p^{2}-m_{c}^{2}\right)^{2}}\right]
$$

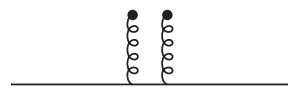

$$
\frac{i \delta_{a b}}{12}\left[\frac{p^{2}+m_{c} \not \not}{\left(p^{2}-m_{c}^{2}\right)^{4}}\right] m_{c}\left\langle g_{s}^{2} G^{2}\right\rangle
$$





\section{Apêndice B}

\section{Algumas Relações Usadas em Regras de Soma}

\section{B.1 Calculando os Traços}

No capítulo 3 em uma dado momento do cálculo da função de correlação pelo Lado da OPE, calculamos alguns traços envolvendo matrizes de Dirac. Abaixo segue uma lista das relações que facilitam o cálculo dos traços que surgem no cálculo da função de correlação.

$$
\begin{gathered}
\operatorname{Tr}\{\hat{1}\}=4 \\
\operatorname{Tr}\left\{\gamma_{\mu} \gamma_{\nu}\right\}=4 g_{\mu \nu} \\
\operatorname{Tr}\left\{\gamma_{5}\right\}=0 \\
\operatorname{Tr}\left\{\gamma_{\mu} \gamma_{\nu} \gamma_{5}\right\}=0 \\
\operatorname{Tr}\left\{\gamma_{\mu} \gamma_{\nu} \gamma_{\rho} \gamma_{\sigma}\right\}=4\left(g_{\mu \nu} g_{\rho \sigma}-g_{\mu \rho} g_{\nu \sigma}+g_{\mu \sigma} g_{\nu \rho}\right)
\end{gathered}
$$




$$
\operatorname{Tr}\left\{\sigma_{\mu \nu}\right\}=0
$$

Traço de número ímpar de matrizes de Dirac $=0$.

\section{B.2 Transformadas de Fourier}

$$
\begin{gathered}
\int d^{4} p \frac{\not p e^{-i p x}}{p^{2}+i \epsilon}=\not x \frac{8 \pi^{2}}{x^{4}}, \\
\int d^{4} p \frac{e^{-i p x}}{p^{2}+i \epsilon}=i \frac{4 \pi^{2}}{x^{2}}, \\
\int d^{4} p \frac{\not p e^{-i p x}}{\left(p^{2}+i \epsilon\right)^{2}}=\not x \frac{2 \pi^{2}}{x^{2}}, \\
\int d^{4} p \frac{e^{-i p x}}{\left(p^{2}+i \epsilon\right)^{2}}=-i \pi^{2} \ln \left(-x^{2}\right),
\end{gathered}
$$

\section{B.3 Álgebra das Matrizes de Dirac}

As matrizes de Dirac na representação de Pauli são:

$$
\gamma^{0}=\left(\begin{array}{cc}
\hat{1} & 0 \\
0 & -\hat{1}
\end{array}\right), \quad \gamma^{i}=\left(\begin{array}{cc}
0 & \sigma^{i} \\
-\sigma^{i} & 0
\end{array}\right),
$$

onde $\sigma^{i}$ são as matrizes de Pauli. As matrizes de Dirac satisfazem as seguintes propriedades:

$$
\begin{aligned}
& \left\{\gamma_{\mu}, \gamma_{\nu}\right\}=2 g_{\mu \nu} \\
& \gamma^{0}=\gamma^{0 T}=\gamma^{0 \dagger}
\end{aligned}
$$




$$
\gamma^{0} \gamma^{\mu} \gamma^{0 \dagger}=\gamma^{\mu \dagger}
$$

$$
\gamma_{\mu} \gamma^{\mu}=4
$$

De acordo com as definições abaixo:

$$
\begin{gathered}
C=i \gamma_{2} \gamma^{0} \\
\sigma_{\mu \nu}=\frac{i}{2}\left[\gamma_{\mu}, \gamma_{\nu}\right] \\
\gamma_{5}=i \gamma^{0} \gamma^{1} \gamma^{2} \gamma^{3} \\
K_{\mu \nu}(x)=\not x \sigma_{\mu \nu}+\sigma_{\mu \nu} \not
\end{gathered}
$$

obtemos as seguintes relações

$$
\begin{gathered}
C^{-1}=C^{T}=C^{\dagger}=-C \\
C \gamma_{\mu}^{T} C^{-1}=-\gamma_{\mu} \\
C \sigma_{\mu \nu}^{T} C^{-1}=-\sigma_{\mu \nu} \\
C K_{\mu \nu}^{T}(x) C^{-1}=K_{\mu \nu}(x) \\
K_{\mu_{\nu}}(x) K^{\mu \nu}(x)=24 x^{2} \\
\left\{\gamma_{\mu}, \gamma_{5}\right\}=0
\end{gathered}
$$




$$
\begin{gathered}
\sigma_{\mu \nu} \gamma^{\alpha} \sigma^{\mu \nu}=0 \\
\sigma_{\mu \nu} \sigma^{\mu \nu}=12
\end{gathered}
$$

\section{B.4 Parametrização de Schwinger $(\gamma)$}

$$
\frac{1}{\left(m^{2}-p^{2}\right)^{n}}=\frac{1}{\Gamma(n)} \int_{0}^{\infty} d \gamma \gamma^{n-1} e^{-\gamma\left(\left(m^{2}-p^{2}\right)\right.}
$$




\section{Apêndice $\mathrm{C}$}

\section{Integrais}

\section{C.1 Integrais no Momento}

As integrais no momento a serem resolvidas são:

$$
\begin{aligned}
& \int d^{4} p \frac{e^{-i p x}}{\left(p^{2}-m^{2}\right)^{n}} \\
& \int d^{4} p \frac{p_{\mu} e^{-i p x}}{\left(p^{2}-m^{2}\right)^{n}}
\end{aligned}
$$

Usando (B.28) a (C.1) é reescrita como

$$
\int d^{4} p \frac{e^{-i p x}}{\left(p^{2}-m^{2}\right)^{n}}=(-1)^{n} \int d^{4} p e^{-i p x} \int d \alpha \frac{\alpha^{n-1}}{\Gamma(n)} e^{-\alpha\left(m^{2}-p^{2}\right)} .
$$

Passando para o espaço euclidiano por meio de uma rotação de Wick, temos

$$
\begin{aligned}
\int d^{4} p \frac{e^{-i p x}}{\left(p^{2}-m^{2}\right)^{n}} & =\frac{(-1)^{n} i}{\Gamma(n)} \int_{0}^{\infty} d \alpha \alpha^{n-1} e^{-\alpha m^{2}} \int d^{4} p_{e} e^{-\alpha p_{e}^{2}+x p_{e}} \\
& =\frac{(-1)^{n} i \pi^{2}}{\Gamma(n)} \int_{0}^{\infty} \frac{d \alpha}{\alpha^{3-n}} e^{-\alpha m^{2}+\frac{x^{2}}{4 \alpha}}
\end{aligned}
$$


Agora vamos resolver a integral dada por (C.2). Novamente, aqui usamos (B.28), com isso obtemos:

$$
\int d^{4} p \frac{p_{\mu} e^{-i p x}}{\left(p^{2}-m^{2}\right)^{n}}=(-1)^{n} \int d^{4} p p_{\mu} e^{-i p x} \int_{0}^{\infty} d \beta \frac{\beta^{n-1}}{\Gamma(n)} e^{-\beta\left(m^{2}-p^{2}\right)} .
$$

Podemos reescrever a integral em $p$ em termos de uma derivada, da seguinte maneira:

$$
\int d^{4} p \frac{p_{\mu} e^{-i p x}}{\left(p^{2}-m^{2}\right)^{n}}=\frac{(-1)^{n} i}{\Gamma(n)} \int_{0}^{\infty} d \beta \beta^{n-1} e^{-\beta m^{2}} \frac{\partial}{\partial x_{\mu}}\left[\int d^{4} p e^{\beta p^{2}-i p x}\right] .
$$

Podemos transformá-la numa integral gaussiana aplicando uma rotação de Wick, deste modo obtemos

$$
\begin{aligned}
\int d^{4} p \frac{p_{\mu} e^{-i p x}}{\left(p^{2}-m^{2}\right)^{n}} & =\frac{(-1)^{n+1}}{\Gamma(n)} \int_{0}^{\infty} d \beta \beta^{n-1} e^{-\beta m^{2}} \frac{\partial}{\partial x_{\mu}}\left[\int d^{4} p e^{-\beta p_{e}^{2}+p_{e} x}\right] \\
& =\frac{(-1)^{n+1} \pi^{2}}{2 \Gamma(n)} \int_{0}^{\infty} \frac{d \beta}{\beta^{3-n}} e^{-\beta m^{2}} \frac{\partial}{\partial x_{\mu}}\left[e^{x^{2} / 4 \beta}\right] .
\end{aligned}
$$

Portanto,

$$
\int d^{4} p \frac{p_{\mu} e^{-i p x}}{\left(p^{2}-m^{2}\right)^{n}}=\frac{(-1)^{n+1} \pi^{2}}{2 \Gamma(n)} \int_{0}^{\infty} \frac{d \beta}{\beta^{4-n}} x_{\mu} e^{-\beta m^{2}+x^{2} / 4 \beta}
$$

\section{C.2 A Integral $I_{n}$}

$I_{n}$ é uma integral do tipo

$$
\int d^{4} x \frac{e^{i q x+\frac{x^{2}(\alpha+\beta)}{4 \alpha \beta}}}{\left(x^{2}\right)^{n}}
$$

Indo para o espaço euclidiano via rotação de Wick, obtemos

$$
\int d^{4} x \frac{e^{i q x+\frac{x^{2}(\alpha+\beta)}{4 \alpha \beta}}}{\left(x^{2}\right)^{n}}=-i \int d^{4} x_{e} \frac{e^{-i q_{e} x_{e}+\frac{x_{e}^{2}(\alpha+\beta)}{4 \alpha \beta}}}{(-1)^{n}\left(x^{2}\right)^{n}} .
$$

O denominador da equação acima $\left(x^{2}\right)^{n}$ pode ser reescrito em termo de uma integral do tipo

$$
\frac{1}{\left(x^{2}\right)^{n}}=\frac{1}{(n-1) !} \int_{0}^{\infty} d \delta e^{-\delta x^{2}} \delta^{n-1}
$$


Assim, substituindo-o no segundo membro de (C.10) segue

$$
I_{n}=\frac{(-1)^{n-1} i}{(n-1) !} \int_{0}^{\infty} d \delta \delta^{n-1} e^{-\delta x_{e}^{2}} \int d^{4} x_{e} e^{-i q_{e} x_{e}-\frac{x_{e}^{2}(\alpha+\beta)}{4 \alpha \beta}} .
$$

Vamos definir a variável $\omega$ como sendo igual a $\omega=\delta+\frac{1}{4 \alpha}+\frac{1}{4 \beta}$. Completando o quadrado obtemos uma integral gaussiana. Logo,

$$
\begin{aligned}
I_{n} & =\frac{(-1)^{n-1} i}{(n-1) !} \int_{0}^{\infty} d \delta \delta^{n-1} e^{-\frac{q_{e}^{2}}{4 \omega}} \underbrace{\int d^{4} x_{e} e^{-b\left(x_{e}+\frac{i q_{e}}{2 b}\right)^{2}}}_{\text {integral gaussiana }} \\
& =\frac{(-1)^{n-1} i \pi^{2}}{(n-1) !} \int_{0}^{\infty} d \delta \frac{\delta^{n-1}}{\omega^{2}} e^{-\frac{q_{e}^{2}}{4 \omega}} .
\end{aligned}
$$

Finalmente fazendo a seguinte mudança de variável: $\delta=\frac{1}{4 \gamma}$, obtemos

$$
I_{n}=\frac{(-1)^{n-1} i \pi^{2}}{4^{n-2}(n-1) !} \int_{0}^{\infty} \frac{d \gamma}{\gamma^{n-1}} \frac{\alpha^{2} \beta^{2}}{(\alpha \beta+\beta \gamma+\gamma \alpha)^{2}} e^{-\frac{\alpha \beta \gamma q_{e}^{2}}{\alpha \beta+\beta \gamma+\gamma \alpha}}
$$

\section{C.3 A Integral $I_{n m k l}$}

A integral $I_{n m k l}$ é dada por

$$
I_{n m k l}=\int_{0}^{\infty} \frac{d \alpha d \beta d \gamma}{\alpha^{n} \beta^{m} \gamma^{k}} \frac{e^{-m_{c}^{2}(\alpha+\beta)-\frac{\alpha \beta \gamma q_{e}^{2}}{\alpha \beta+\beta \gamma+\gamma \alpha}}}{(\alpha \beta+\beta \gamma+\gamma \alpha)^{l}}
$$

Podemos reescrevê-la da seguinte maneira:

$$
I_{n m k l}=\int_{0}^{\infty} \frac{d \alpha d \beta d \gamma}{\alpha^{n} \beta^{m} \gamma^{k+l}} \frac{e^{-m_{c}^{2}(\alpha+\beta)-\frac{\alpha \beta \gamma q_{e}^{2}}{\alpha \beta / \gamma+\beta \gamma+\gamma \alpha}}}{(\alpha \beta / \gamma+\beta+\alpha)^{l}}
$$

Fazendo a mudança de variáveis: $\alpha=\alpha^{\prime}, \beta=\beta^{\prime}$ e $\frac{\alpha \beta}{\gamma}=\gamma^{\prime}$, temos:

$$
I_{n m k l}=\int_{0}^{\infty} \frac{d \alpha^{\prime} d \beta^{\prime} d \gamma^{\prime}}{\alpha^{\prime n+k+l-1} \beta^{\prime} m+k+l-1} \frac{\gamma^{\prime l+k-2}}{\left(\alpha^{\prime}+\beta^{\prime}+\gamma^{\prime}\right)^{l}} e^{-m_{c}^{2}\left(\alpha^{\prime}+\beta^{\prime}\right)-\frac{\alpha^{\prime} \beta^{\prime} \gamma^{\prime} q_{e}^{2}}{\alpha^{\prime}+\beta^{\prime}+\gamma^{\prime}}}
$$

Inserindo a identidade

$$
1=\int_{0}^{\infty} d \lambda \delta\left[\lambda-\left(\alpha^{\prime}+\beta^{\prime}+\gamma^{\prime}\right)\right]
$$


obtemos

$I_{n m k l}=\int_{0}^{\infty} \frac{d \alpha^{\prime} d \beta^{\prime} d \gamma^{\prime}}{\alpha^{\prime n+k+l-1} \beta^{\prime m+k+l-1}} \frac{\gamma^{\prime l+k-2}}{\left(\alpha^{\prime}+\beta^{\prime}+\gamma^{\prime}\right)^{l}} e^{-m_{c}^{2}\left(\alpha^{\prime}+\beta^{\prime}\right)-\frac{\alpha^{\prime} \beta^{\prime} \gamma^{\prime} q_{e}^{2}}{\alpha^{\prime}+\beta^{\prime}+\gamma^{\prime}}} \int_{0}^{\infty} d \lambda \delta\left[\lambda-\left(\alpha^{\prime}+\beta^{\prime}+\gamma^{\prime}\right)\right]$.

Vamos fazer uma mudança de escala do tipo abaixo, omitindo o símbolo ' nas variáveis: $\alpha=\lambda \alpha, \beta=\lambda \beta$ e $\gamma=\lambda \gamma$, assim, resulta que

$$
I_{n m k l}=\int_{0}^{\infty} \frac{d \alpha d \beta d \gamma d \lambda \delta[1-(\alpha+\beta+\gamma)]}{\alpha^{n+k+l-1} \beta^{m+k+l-1} \lambda^{n+m+k+2 l-2}} \frac{\gamma^{l+k-2}}{(\alpha+\beta+\gamma)^{l}} e^{-m_{c}^{2}(\alpha+\beta)-\frac{\alpha \beta \gamma q_{e}^{2}}{\alpha+\beta+\gamma}} .
$$

Integrando na variável $\gamma$ por meio da delta, chegamos ao resultado abaixo

$$
I_{n m k l}=\int_{0}^{1} \frac{d \alpha d \beta \theta(1-\alpha-\beta)}{\alpha^{n+k+l-1} \beta^{m+k+l-1}}(1-\alpha-\beta)^{l+k-2} \int_{0}^{\infty} d \lambda \frac{e^{-\lambda\left[m_{c}^{2}(\alpha+\beta)+\alpha \beta q_{e}\right]}}{\lambda^{n+m+k+2 l-2}} .
$$

\section{C.4 A Integral $I_{\lambda}^{n}$}

A integral $I_{\lambda}^{n}$ é uma integral do tipo

$$
I_{\lambda}^{n}=\int \frac{d \lambda}{\lambda^{n}} e^{-\lambda f}
$$

onde $f$ é uma função positiva. Considere inicialmente a integral

$$
I_{\lambda}^{0}=\int_{0}^{\infty} d \lambda e^{-\lambda f}=\frac{1}{f}
$$

Podemos reescrevê-la da seguinte maneira

$$
\int_{0}^{\infty} d \lambda e^{-\lambda f}=-\frac{\partial}{\partial f} \int_{0}^{\infty} \frac{d \lambda}{\lambda} e^{-\lambda f}
$$

Desse modo, ao integrarmos em $f$ ambos os membros, obtemos

$$
\begin{aligned}
I_{\lambda}^{1}=\int_{0}^{\infty} \frac{d \lambda}{\lambda} e^{-\lambda f} & =-\int d f \int_{0}^{\infty} d \lambda e^{-\lambda f} \\
& =-\int \frac{d f}{f} \\
& =-\ln f+c .
\end{aligned}
$$


Que é o resultado para a integral (C.21) para $n=1$. Note que a divergência está na constante $c$. Entretanto, nas Regras de Soma da QCD estamos interessado na parte imaginária das integrais. Seguindo o raciocínio, encontramos o resultado para $n=2$

$$
\begin{aligned}
I_{\lambda}^{2}=\int_{0}^{\infty} \frac{d \lambda}{\lambda^{2}} e^{-\lambda f} & =\int d f \ln f \\
& =f \ln f-f+c t e .
\end{aligned}
$$

Novamente, estamos interessados na parte $f \ln f$ visto que somente ela pode ter parte imaginária. Prosseguindo agora para $n=3, n=4$ e $n=5$, temos

$$
\begin{gathered}
I_{\lambda}^{3}=\int_{0}^{\infty} \frac{d \lambda}{\lambda^{3}} e^{-\lambda f}=-\frac{f^{2} \ln f}{2}+\ldots \\
I_{\lambda}^{4}=\int_{0}^{\infty} \frac{d \lambda}{\lambda^{4}} e^{-\lambda f}=\frac{f^{3} \ln f}{3 !}+\ldots \\
I_{\lambda}^{5}=\int_{0}^{\infty} \frac{d \lambda}{\lambda^{5}} e^{-\lambda f}=-\frac{f^{4} \ln f}{4 !}+\ldots
\end{gathered}
$$

Com isso, por indução chegamos ao resultado abaixo

$$
I_{\lambda}^{n}=\int_{0}^{\infty} \frac{d \lambda}{\lambda^{n}} e^{-\lambda f}=\frac{(-1)^{n}}{(n-1) !} f^{n-1} \ln f+O\left(f^{n}\right) .
$$

\section{C.5 Cálculo dos Diagramas Perturbativos}

A integral que temos que resolver para encontrarmos a contribuição dos diagramas perturbativos para a densidade espectral são dadas pelas Eq.s (3.20) e (3.22). Vamos definir o primeiro termo de (3.20) como $I_{1}$, dado por

$$
I_{1}=\frac{9 i}{2^{7} \pi^{12}} \int d^{4} x d^{4} p_{1} d^{4} p_{2} \frac{e^{i q x-i\left(p_{1}+p_{2}\right) x}}{x^{8}\left(p_{1}^{2}-m_{c}^{2}\right)\left(p_{2}^{2}-m_{c}^{2}\right)} m_{c}^{2} x_{\mu} x_{\nu} .
$$


Usando (C.4) tomando $n=1$ e substituindo o resultado em (C.30), temos

$$
\begin{aligned}
I_{1} & =\frac{9 i m_{c}^{2}}{2^{7} \pi^{12}} \int d^{4} x \frac{e^{i q x} x_{\mu} x_{\nu}}{x^{8}} \int_{0}^{\infty} \frac{d \alpha d \beta}{\alpha^{2} \beta^{2}} e^{-m_{c}^{2}(\alpha+\beta)+x^{2}(1 / 4 \alpha+1 / 4 \beta)} \\
& =\frac{9 i m_{c}^{2}}{2^{7} \pi^{12}} \underbrace{\int d^{4} x \frac{x_{\mu} x_{\nu} e^{i q x+\frac{x^{2}(\alpha+\beta)}{4 \alpha \beta}}}{x^{8}}}_{I_{\mu \nu}} \int_{0}^{\infty} \frac{d \alpha d \beta}{\alpha^{2} \beta^{2}} e^{-m_{c}^{2}(\alpha+\beta)} .
\end{aligned}
$$

Para calcularmos $I_{\mu \nu}$ usamos:

$$
\begin{aligned}
I_{\mu \nu} & =-\int d^{4} x \frac{\partial^{2}}{\partial q_{\mu} \partial q_{\nu}} \frac{e^{i q x+\frac{x^{2}(\alpha+\beta)}{4 \alpha \beta}}}{x^{8}} \\
& =-\frac{\partial^{2}}{\partial q_{\mu} \partial q_{\nu}} \underbrace{\int d^{4} x \frac{e^{i q x+\frac{x^{2}(\alpha+\beta)}{4 \alpha \beta}}}{x^{8}}}_{I_{n=4}} .
\end{aligned}
$$

Tomando $n=4$ na (C.14) e substituindo o resultado na (C.32), encontramos:

$$
I_{\mu \nu}=\frac{i \pi^{2}}{3.2^{5}} \frac{\partial^{2}}{\partial q_{\mu} \partial q_{\nu}} \int_{0}^{\infty} \frac{d \gamma}{\gamma^{3}} \frac{\alpha^{2} \beta^{2}}{(\alpha \beta+\beta \gamma+\gamma \alpha)^{2}} e^{-\frac{\alpha \beta \gamma q_{e}^{2}}{\alpha \beta+\beta \gamma+\gamma \alpha}}
$$

Assim substituindo esse resultado em (C.30) temos

$$
I_{1}=\frac{9 m_{c}^{2}}{2^{12} .3 \pi^{6}} \int_{0}^{\infty} \frac{d \alpha d \beta d \gamma}{\gamma^{3}} \frac{\partial^{2}}{\partial q_{\mu} \partial q_{\nu}} \frac{e^{-m_{c}^{2}(\alpha+\beta)+\frac{\alpha \beta \gamma q^{2}}{\alpha \beta+\beta \gamma+\gamma \alpha}}}{(\alpha \beta+\beta \gamma+\gamma \alpha)^{2}}
$$

Como a integração é realizada sobre as variáveis $\alpha, \beta$ e $\gamma$, podemos permutar a integral com a derivada $\frac{\partial^{2}}{\partial q_{\mu} \partial q_{\nu}}$, assim

$$
I_{1}=\frac{9 m_{c}^{2}}{2^{12} .3 \pi^{6}} \frac{\partial^{2}}{\partial q_{\mu} \partial q_{\nu}} \underbrace{\int_{0=3}^{\infty} \frac{d \alpha d \beta d \gamma}{\gamma^{3}} \frac{e^{-m_{c}^{2}(\alpha+\beta)+\frac{\alpha \beta \gamma q^{2}}{\alpha \beta+\beta \gamma+\gamma \alpha}}}{(\alpha \beta+\beta \gamma+\gamma \alpha)^{2}}}_{I_{n=m=0}} .
$$

Tomando os valores: $n=0, m=0, k=3$ e $l=2$ na (C.20), segue

$$
I_{0032}=\int_{0}^{1} \frac{d \alpha d \beta \theta(1-\alpha-\beta)}{\alpha^{4} \beta^{4}}(1-\alpha-\beta)^{3} \int_{0}^{\infty} d \lambda \frac{e^{-\lambda\left[m_{c}^{2}(\alpha+\beta)+\alpha \beta q_{e}\right]}}{\lambda^{5}}
$$


Definindo $F(\alpha, \beta)=-\lambda\left[m_{c}^{2}(\alpha+\beta)+\alpha \beta q_{e}\right]$ e usando (C.36) em (C.35), chegamos ao seguinte resultado:

$$
\begin{aligned}
I_{1} & =\frac{9 m_{c}^{2}}{2^{12} .3 \pi^{6}} \frac{\partial^{2}}{\partial q_{\mu} \partial q_{\nu}} \int_{0}^{1} \frac{d \alpha d \beta \theta(1-\alpha-\beta)}{\alpha^{4} \beta^{4}}(1-\alpha-\beta)^{3} \int_{0}^{\infty} d \lambda \frac{e^{-\lambda F(\alpha, \beta)}}{\lambda^{5}} \\
& =\frac{9 m_{c}^{2}}{2^{12} .3 \pi^{6}} \int_{0}^{1} \frac{d \alpha d \beta \theta(1-\alpha-\beta)}{\alpha^{4} \beta^{4}}(1-\alpha-\beta)^{3} \int_{0}^{\infty} d \lambda \underbrace{\frac{\partial^{2}}{\partial q_{\mu} \partial q_{\nu}} \frac{e^{-\lambda F(\alpha, \beta)}}{\lambda^{5}}}_{D}
\end{aligned}
$$

Resolvendo $D$, segue que

$$
\begin{aligned}
D & =\frac{\partial^{2}}{\partial q_{\mu} \partial q_{\nu}} \frac{e^{-\lambda F(\alpha, \beta)}}{\lambda^{5}} \\
& =\frac{e^{-\lambda F(\alpha, \beta)}}{\lambda^{5}}\left[(2 \lambda \alpha \beta) g_{\mu \nu}+\left(4 \lambda^{2} \alpha^{2} \beta^{2}\right) q_{\mu} q_{\nu}\right] .
\end{aligned}
$$

Estamos interessados somente no coeficiente da estrutura $g_{\mu \nu}$. Portanto, (C.37) fica

$$
I_{1}=\frac{9 m_{c}^{2}}{2^{11} .3 \pi^{6}} \int_{0}^{1} \frac{d \alpha d \beta \theta(1-\alpha-\beta)}{\alpha^{3} \beta^{3}}(1-\alpha-\beta)^{3} \int_{0}^{\infty} d \lambda \frac{e^{-\lambda F(\alpha, \beta)}}{\lambda^{4}} .
$$

A integral na variável $\lambda$ pode ser resolvida usando o resultado (C.29) tomando $n=4$, com isso temos

$$
I_{1}=\frac{9 m_{c}^{2}}{2^{11} .3 \pi^{6}} \int_{0}^{1} \frac{d \alpha d \beta \theta(1-\alpha-\beta)}{\alpha^{3} \beta^{3}}(1-\alpha-\beta)^{3} \frac{(-1)^{4} F^{3}(\alpha, \beta)}{3 !} \ln F(\alpha, \beta) .
$$

Agora vamos resolver o segundo termo de (3.20) dado por

$$
I_{2}=\frac{-9 i}{2^{7} \pi^{12}} \int d^{4} x d^{4} p_{1} d^{4} p_{2} \frac{e^{i q x-i\left(p_{1}+p_{2}\right) x}}{x^{8}\left(p_{1}^{2}-m_{c}^{2}\right)\left(p_{2}^{2}-m_{c}^{2}\right)}\left(x \cdot p_{1}\right)\left(x . p_{2}\right) g_{\mu \nu} .
$$

Podemos reescrevê-la da seguinte maneira:

$$
\begin{aligned}
I_{2} & =\frac{-9 i}{2^{7} \pi^{12}} \int \frac{d^{4} x e^{i q x}}{x^{8}} \int \frac{d^{4} p_{1} e^{-i p_{1} x}\left(x_{\alpha} p_{1}^{\alpha}\right)}{\left(p_{1}^{2}-m_{c}^{2}\right)} \int \frac{d^{4} p_{2} e^{-i p_{2} x}\left(x_{\beta} p_{2}^{\beta}\right)}{\left(p_{2}^{2}-m_{c}^{2}\right)} g_{\mu \nu} \\
& =\frac{-9 i}{2^{7} \pi^{12}} \int \frac{d^{4} x e^{i q x}}{x^{8}} x_{\alpha} x_{\beta} g_{\mu \nu} \int \frac{d^{4} p_{1} p_{1}^{\alpha} e^{-i p_{1} x}}{\left(p_{1}^{2}-m_{c}^{2}\right)} \int \frac{d^{4} p_{2} p_{2}^{\beta} e^{-i p_{2} x}}{\left(p_{2}^{2}-m_{c}^{2}\right)} .
\end{aligned}
$$

Fazendo $n=1$ na (C.8) e substituindo o resultado na (C.42), resulta em

$$
I_{2}=\frac{-9 i}{2^{7} \pi^{8}} \underbrace{\int \frac{d^{4} x e^{i q x+\frac{(\alpha+\beta) x^{2}}{4 \alpha \beta}}}{x^{4}}}_{I_{n=2}} \int_{0}^{\infty} \frac{d \alpha d \beta}{\alpha^{3} \beta^{3}} e^{-m_{c}^{2}(\alpha+\beta)} .
$$


Fazendo $n=2$ na (C.14) e substituindo na equação acima, $I_{2}$ fica

$$
I_{2}=\frac{-9}{2^{9} \pi^{6}} \int_{0}^{\infty} \frac{d \alpha d \beta d \gamma}{\alpha \beta \gamma} \frac{e^{-m_{c}^{2}(\alpha+\beta)-\frac{\alpha \beta \gamma q_{e}^{2}}{(\alpha \beta+\beta \gamma+\gamma \alpha)}}}{(\alpha \beta+\beta \gamma+\gamma \alpha)^{2}}
$$

Deste modo, fazendo $n=1, m=1, k=1$ e $l=2$ em (C.20), resultará na solução de (C.44):

$$
I_{2}=\frac{-9}{2^{9} \pi^{6}} \int_{0}^{1} \frac{d \alpha d \beta \theta(1-\alpha-\beta)(1-\alpha-\beta)}{\alpha^{3} \beta^{3}} \int_{0}^{\infty} \frac{d \lambda}{\lambda^{5}} e^{-\lambda F(\alpha, \beta)} .
$$

A integral em $\lambda$ é resolvida fazendo $n=5$ na (C.29), portanto, segue que o resultado para $I_{2}$ é dado por

$$
I_{2}=\frac{9}{2^{12} \pi^{6} 3} \int_{0}^{1} \frac{d \alpha d \beta \theta(1-\alpha-\beta)(1-\alpha-\beta)}{\alpha^{3} \beta^{3}} F^{4}(\alpha, \beta) \ln F(\alpha, \beta) .
$$

Finalmente, o terceiro termo da (C.35) contém integrais semelhantes aos dois primeiros termos. Assim, $I_{3}$ resultará

$$
I_{3}=-\frac{9}{2^{12} \pi^{6} 3} \int_{0}^{1} \frac{d \alpha d \beta \theta(1-\alpha-\beta)(1-\alpha-\beta)^{2}}{\alpha^{3} \beta^{3}} F^{4}(\alpha, \beta) \ln F(\alpha, \beta) .
$$

Portanto, a solução de (3.20) será a soma de $I_{1}+I_{2}+I_{3}$. Após realizarmos a soma e reorganizar os termos, obtemos:

$$
\Pi^{\text {pert }}(q)=-\frac{1}{2^{12} \pi^{6}} \int_{0}^{1} \frac{d \alpha \beta}{\alpha^{3} \beta^{3}} F^{3}(\alpha, \beta)\left[2 m_{c}^{2}(1-\alpha-\beta)^{2}+3 F(\alpha, \beta)(1+\alpha+\beta)\right] \ln F(\alpha, \beta) .
$$

Portanto, usando o resultado apresentado na Seção 3.2 do Capítulo 1, a equação acima pode ser escrita em termos de uma relação de dispersão

$$
\begin{gathered}
\rho^{\text {pert }}(s)=\frac{1}{2^{12} \pi^{6}} \int_{\alpha_{\min }}^{\alpha_{\max }} \frac{d \alpha}{\alpha^{3}} \int_{\beta_{\min }}^{1-\alpha} \frac{d \beta}{\beta^{3}} F^{3}(\alpha, \beta)\left[2 m_{c}^{2}(1-\alpha-\beta)^{2}+\right. \\
+3 F(\alpha, \beta)(1+\alpha+\beta)] .
\end{gathered}
$$




\section{Referências Bibliográficas}

[1] M. Gell-Mann, Phys. Rev. Lett. 8:214-2 (1964).

[2] G. Zweig, CERN Preprint 8182/TH401: 24 (1964).

[3] S. L. Choi et al. Phys. Rev. Lett. 91262001 (2003); V. M. Abazov et al. (D0), Phys. Rev. Lett. 93, 162002; D. Acosta et al. [CDFII], Phys. Rev. Lett. 93, 072001; ); B. Aubert et al. (BaBar), Phys. Rev. D 71, 071103 (2005).

[4] B.-A. Li, Phys. Lett. B 605, 306 (2005).

[5] D. Bugg, Phys. Lett. B 598, 8 (2004).

[6] D. Abulencia, et al., [CDF Collaboration], Phys. Rev. Lett. 98 (2007) 132002.

[7] P. del Amo Sanches, et al., [BaBar Collaboration]. arXiv:1005.5190.

[8] G. Gokhroo, et al., [Belle Collaboration], Phys. Rev. Lett. 97 (2006) 162002.

[9] B. Aubert, et al., [BaBar Collaboration], Phys. Rev. D 77 (2008) 011102 .

[10] L. Maiani et al, Phys. Rev. D 72, 031502 (2005).

[11] R.M. Albuquerque, M. Nielsen, Nucl. Phys. A 815 (2009) 53, arXiv:0804.4817. 
[12] K. Abe et al. [Belle Collaboration], Phys. Rev. Lett. 100, 142001 (2008) [arXiv:0708.1790].

[13] S.H. Lee, M. Nielsen, U. Wiedner, arXiv:0803.1168.

[14] T. Aaltonen et al. [CDF Collaboration], Phys. Rev. Lett. 102, 242002 (2009) [arXiv:09032229].

[15] X. Liu and S.-L. Zhu, arXiv:0903.2529.

[16] S.-K. Choi et al. [Belle Collaboration], Phys. Rev. Lett. 94, 182002 (2005); B. Albert et al. [BaBar Collaboration], Phys. Rev. Lett. 101, 082001 (2008).

[17] N. Mahajan, arXiv:0903.3107.

[18] T. Branz, T. Gutsche and V.E. Lyubovitskij, Phys. Rev. D 80, 054019 (2009).

[19] C.P. Shen et al. [Belle Collaboration], arXiv:0912.2383.

[20] R.M. Albuquerque, J.M. Dias, M. Nielsen, Phys. Lett. B690, 141 (2010).

[21] M.A. Shifman, A.I. Vainshtein e V.I. Zakharov, Nucl. Phys. B147, 385 (1979).

[22] V.M. Belyaev, V.M. Braun, A. Khodjamirian e R. Ruckl, Phys. Rev. D51, 6177 (1995).

[23] M.E. Peskin e D.V. Schroeder, An Introduction to Quantum Field Theory, Addison-Wesley (1995).

[24] R.D. Matheus, Partículas Exóticas em Regras de Soma da QCD, Tese de Doutorado, Instituto de Física - Universidade de São Paulo (2006). 
[25] R.M. Albuquerque, Estudo da Estrutura dos Novos Mésons, Tese de Mestrado, Instituto de Física - Universidade de São Paulo (2008).

[26] M. Nielsen, F.S. Navarra e S.H. Lee, Phys. Rept. (2010).

[27] P. Colangelo e A. Khodjamirian, arXiv:hep-ph/0010175 (2000).

[28] K. G. Wilson, Phys. Rev. 179, 1499 (1969).

[29] C.P. Shen, et al., Belle Collaboration, arXiv:0912.2383.

[30] Fl. Stancu, arXiv:0906.2485.

[31] Silvan S. Schweber, An a Introduction to Relativistic Quantum Field Theory, Dover (1961).

[32] L.J. Reinders, H. Rubinstein e S. Yazaki, Phys. Rept. 127, 1 (1985).

[33] S.-K. Choi et al. [Belle Collaboration], Phys. Rev. Lett. 91, 262001 (2003).

[34] M. Nielsen, F.S. Navarra, S.H. Lee, arXiv:0911.1958.

[35] X. Liu and S.-L. Zhu, Phys. Rev. D 80, 017502 (2009).

[36] N. Mahajan, Phys. Lett. B 679, 228

[37] R.M. Albuquerque, M.E. Bracco and M. Nielsen, Phys. Lett. B 678, 186 (2009).

[38] G.-J. Ding, Eur. Phys. J. C 64, 297 (2009).

[39] J.R. Zhang and M. Q. Huang, arXiv:0905.4178.

[40] X. Liu and H.W. Ke, Phys. Rev. D 80, 034009 (2009).

[41] R. Molina and E. Oset, arXiv:0907.3043.

[42] Fl. Stancu, arXiv:0906.2485. 
[43] J.R. Zhang and M. Q. Huang, arXiv:0905.4672.

[44] R.M. Albuquerque and M. Nielsen, Nucl. Phys. A815, 53 (2009) [arXiv:0804.4817].

[45] X. Liu, G.Z. Luo and Z.F. Sun, arXiv:0911.3694.

[46] Z.-G. Wang, arXiv:0912.4626.

Nucl. Phys. B147, 385 (1979).

127, 1 (1985).

[47] For a review and references to original works, see e.g., S. Narison, QCD as a theory of hadrons, Cambridge Monogr. Part. Phys. Nucl. Phys. Cosmol. 17, 1 (2002) [hep-h/0205006]; QCD spectral sum rules , World Sci. Lect. Notes Phys. 26, 1 (1989); Acta Phys. Pol. B26, 687 (1995); Riv. Nuov. Cim. 10N2, 1 (1987); Phys. Rept. 84, 263 (1982).

[48] B. L. Ioffe, Nucl. Phys. B188, 317 (1981); B191, 591(E) (1981).

[49] S. Narison, Phys. Lett. B466, 345 (1999); S. Narison, Phys. Lett. B361, 121 (1995); S. Narison, Phys. Lett. B387, 162 (1996); S. Narison, Phys. Lett. B624, 223 (2005).

[50] R.D. Matheus et al., Phys. Rev. D75, 014005 (2007).

[51] S.H. Lee, A. Mihara, F.S. Navarra and M. Nielsen, Phys. Lett. B661, 28 (2008) [arXiv:0710.1029].

[52] M.E. Bracco, S.H. Lee, M. Nielsen, R. Rodrigues da Silva, Phys. Lett. B671, 240 (2009) [arXiv:0807.3275].

[53] S.H. Lee, K. Morita and M. Nielsen, Nucl. Phys. A815, 29 (2009) [arXiv:0808.0690]. 
[54] S.H. Lee, K. Morita and M. Nielsen, Phys. Rev. D78, 076001 (2008) [arXiv:0808.3168]. 